\title{
A Hybrid Whale Optimization with Seagull Algorithm for Global Optimization Problems
}

\author{
Yanhui Che $\mathbb{D}$ and Dengxu He \\ School of Mathematics and Physics, Guangxi University for Nationalities, Nanning 530006, China \\ Correspondence should be addressed to Dengxu He; dengxuhe@126.com
}

Received 13 October 2020; Revised 2 November 2020; Accepted 8 January 2021; Published 29 January 2021

Academic Editor: George A. Papakostas

Copyright (c) 2021 Yanhui Che and Dengxu He. This is an open access article distributed under the Creative Commons Attribution License, which permits unrestricted use, distribution, and reproduction in any medium, provided the original work is properly cited.

\begin{abstract}
Seagull optimization algorithm (SOA) inspired by the migration and attack behavior of seagulls in nature is used to solve the global optimization problem. However, like other well-known metaheuristic algorithms, SOA has low computational accuracy and premature convergence. Therefore, in the current work, these problems are solved by proposing the modified version of SOA. This paper proposes a novel hybrid algorithm, called whale optimization with seagull algorithm (WSOA), for solving global optimization problems. The main reason is that the spiral attack prey of seagulls is very similar to the predation behavior of whale bubble net, and the WOA has strong global search ability. Therefore, firstly, this paper combines WOA's contraction surrounding mechanism with SOA's spiral attack behavior to improve the calculation accuracy of SOA. Secondly, the levy flight strategy is introduced into the search formula of SOA, which can effectively avoid premature convergence of algorithms and balance exploration and exploitation among algorithms more effectively. In order to evaluate the effectiveness of solving global optimization problems, 25 benchmark test functions are tested, and WSOA is compared with seven famous metaheuristic algorithms. Statistical analysis and results comparison show that WSOA has obvious advantages compared with other algorithms. Finally, four engineering examples are tested with the proposed algorithm, and the effectiveness and feasibility of WSOA are verified.
\end{abstract}

\section{Introduction}

Over the past ten years, the metaheuristic algorithm has become very popular. For example, it has been widely used to find solutions to many complex problems in engineering and computer science. The main reasons for its popularity are flexibility, gradient-free mechanism, and avoiding falling into local optimization. In other words, metaheuristic algorithms only need to look at the input and output to consider optimization problems and do not need to calculate the derivative of search space, which makes them more flexible in solving various optimization problems.

Compared with traditional optimization algorithms, the metaheuristic algorithm can solve various optimization problems more effectively. Inspired by the intelligent behaviors and evolution laws of natural swarm, this kind of algorithm has attracted the attention of scholars, so many well-known swarm intelligence algorithms have been proposed. For example, Genetic Algorithm (GA) [1] is a random search algorithm, which mainly imitates the natural selection in the biological world. The main reason for the success of GA is that the behaviors of selection, replication, and mutation are random, which can help the algorithm avoid falling into local optimum. Particle Swarm Optimization (PSO) algorithm [2] simulates the foraging behaviors of birds or fish in nature. Ant Colony Optimization (ACO) algorithm [3] is inspired by the behaviors of ants to find the shortest path to food. Grey Wolf Optimization (GWO) algorithm [4] simulates the hierarchical mechanism and predation behaviors of grey wolf population in nature. Moth-Flame Optimization (MFO) algorithm [5] is mainly derived from the simulation of the lateral positioning flight mode of moths. Whale Optimization Algorithm (WOA) [6] simulates the predatory behaviors of humpback whale populations in nature. Squirrel Search Algorithm (SqSA) [7] is mainly inspired by squirrel's foraging behavior. 
The inspiration of Sea Lion Optimization (SLnO) algorithm [8] mainly comes from the hunting behavior of sea lions. Bald Eagle Search (BES) [9] is mainly inspired by vulture hunting strategy. Chimp optimization algorithm (COA) [10] is inspired by the behavior of chimpanzee's individual intelligence and sexual motivation in hunting. Artificial Electric Field Algorithm (AEFA) [11] is mainly inspired by Coulomb's electrostatic force law and Newton's law of motion. Invasive Weed Optimization (IWO) [12] algorithm is mainly inspired by the process of weed invasion in nature. Artificial Bee Colony (ABC) [13] simulates the hunting honey behaviors of bees in nature.

Although each metaheuristic algorithm has its own unique advantages, according to the theorem of no free lunch [14], no algorithm can solve all optimization problems [15]. The performance of the metaheuristic algorithm mainly depends on its exploration and exploitation ability. Therefore, many researchers constantly propose new algorithms and improve the original algorithm. For example, in order to improve the searching ability of grey wolves, Gupta and Deep proposed a novel random walk grey wolf optimizer in 2018 [16]. In 2019, the improved SSA tried to balance the exploration and exploitation of the algorithm to avoid the disadvantages of suboptimal solution and low accuracy [17]. In 2020, a new grey wolf optimization algorithm based on memory was proposed [18]. In 2020, a memory-guided sine and cosine algorithm was improved [19]. In 2020, an Archimedes Optimization Algorithm (AOA) was proposed to solve the optimization problem [20]. Hussien et al. proposed a new binary whale optimization algorithm for discrete optimization problems in 2020 [21]. Among them, combining two or more metaheuristic algorithms into a hybrid algorithm has attracted more and more attention in the field of optimization algorithms. For example, Gupta and Deep proposed a hybrid adaptive sine and cosine algorithm based on reverse learning in 2019 to solve the defects of the original algorithm such as low diversity and local optimal stagnation [22]. In 2019, a hybrid grey wolf optimizer with mutation operator was proposed to avoid the algorithm falling into local optimal solution [23]. Compared with the single algorithm, the hybrid algorithm can find the global optimal solution more effectively. In this paper, the contraction surrounding mechanism of WOA is combined with the spiral attack behaviors of SOA in order to obtain better performance.

Inspired by seagull migration and attack behaviors in nature, Indian scholar Gaurav Dhiman proposed a novel bionic algorithm called seagull optimization algorithm (SOA) in 2019 [24]. The most important things about seagulls are their migration and attack behaviors. Migration behavior is that seagulls migrate from one place to another full of food. Attack behavior is that seagulls often attack other birds at sea during migration, and they will attack their prey in a spiral natural shape. Since the migration behaviors of seagulls are a large-scale flight, they are regarded as global search. In contrast, the attack behaviors of seagulls are considered as local search. Therefore, SOA based on simulating seagull migration and attack behaviors is also regarded as global search and local searching process.
In recent years, scholars have conducted more extensive research on SOA. Jiang et al. studied a hybrid classification method based on the oppositional seagull optimization algorithm [25]. Jia et al. proposed a new hybrid seagull optimization algorithm for feature selection [26]. Dhiman et al. studied Emo SOA: a new evolutionary multitarget gull global optimization algorithm [27]. Panagant et al. studied the seagull optimization algorithm to solve the design optimization problem in the real world [28].

Based on the characteristics of SOA and WOA, a hybrid whale optimization with seagull algorithm (WSOA) is proposed to improve the potential of SOA. In view of the shortcomings of SOA, such as premature convergence and low computational accuracy, this paper combines the contraction surrounding mechanism of whale optimization algorithm with the spiral attack behavior of seagull optimization algorithm to improve the computational accuracy of SOA. The core idea of this hybrid technology is to make use of the strong global search ability of WOA and the fast convergence ability of SOA and effectively accelerate the search ability of WSOA. In addition, the levy flight strategy is introduced into the search formula of SOA, which can effectively avoid premature convergence of algorithms and balance exploration and exploitation among algorithms more effectively.

The main contributions of this paper can be summarized as follows: Firstly, a new hybrid whale optimization with seagull algorithm (WSOA) for optimization problems is proposed. WSOA takes advantage of the strong global search ability of WOA and the fast convergence ability of SOA. In addition, the levy flight strategy is introduced into the search formula of SOA, which can effectively avoid premature convergence of algorithms and balance exploration and exploitation among algorithms more effectively. Secondly, in order to verify the performance of WSOA, this paper verifies 25 benchmark test functions. Finally, the proposed WSOA is used to solve four constrained engineering optimization problems, which show that the proposed algorithm has strong performance.

The rest of this paper is structured as follows: Section 2 briefly introduces the original seagull optimization algorithm; Section 3 briefly introduces the original whale optimization algorithm; Section 4 introduces the hybrid whale optimization with seagull algorithm (WSOA); Section 5 is the experimental results and analysis; Section 6 introduces the limitations of WSOA; Section 7 is the conclusion of this paper and future work.

\section{Seagull Optimization Algorithm (SOA)}

Seagull optimization algorithm mainly simulates the migration and attack behaviors of seagulls in nature. Seagulls are a kind of seabird all over the world, with various species, which mainly feed on insects, fish, reptiles, amphibians, and earthworms. Seagulls are very clever birds that use their wisdom to find food and attack prey. For example, they use breadcrumbs to attract fish and their feet to imitate the sound of rain to lure earthworms hidden underground. Migration and attack are the most important behaviors of 
seagulls. Migration behaviors are defined as the source of food for seagulls. Attack behaviors are defined as the attack behaviors from the seagulls against the migrating birds at sea.

During the migration process, the algorithm simulates how seagulls move from one place to another. At this stage, seagulls must meet the following conditions.

In order to avoid collisions between seagulls and adjacent seagulls, variable $\mathrm{A}$ is added to calculate the new search agent position.

$$
\vec{C}_{s}=A \times \vec{P}_{s}(x),
$$

where $\vec{C}_{s}$ represents the location of the search agent that does not collide with other search agents, $\vec{P}_{s}$ represents the current location of the search agent, $x$ represents the current iteration times, and $A$ represents the mobile behavior of the search agent in a given search space.

$$
A=f_{c}-\left(x \times\left(\frac{f_{c}}{\text { Max }_{\text {iteration }}}\right)\right)
$$

where: $x=0,1,2, \ldots, \operatorname{Max}_{\text {iteration, }}$

where $f_{c}$ is introduced to control the use frequency of variable $A$, which is linearly reduced from $f_{c}$ to 0 , and in this paper $f_{c}$ is set to 2 .

After avoiding collisions between seagulls, the search agent moves in the direction of the best neighbor.

$$
\vec{M}_{s}=B \times\left(\vec{P}_{b s}(x)-\vec{P}_{s}(x)\right),
$$

where $\vec{M}_{s}$ represents the position of search agent $\vec{P}_{s}$ towards the best-fit search agent $\vec{P}_{b s}$ (i.e., the best seagull with a small fitness value). The behavior of $B$ is randomized, which is responsible for proper balancing between exploration and exploitation. $B$ is calculated as

$$
B=2 \times A^{2} \times \mathrm{rd}
$$

where $\mathrm{rd}$ is a random number that lies in the range of $[0,1]$.

Finally, the search agent can update its position relative to the best search agent.

$$
\vec{D}_{s}=\left|\vec{C}_{s}+\vec{M}_{s}\right|
$$

where $\vec{D}_{s}$ represents the distance between the search agent and best-fit search agent (i.e., best seagull whose fitness value is less).

In the process of attacking prey, seagulls will spiral motion behavior in the air. This behavior in $x, y$, and $z$ planes is described as follows:

$$
\begin{aligned}
& x^{\prime}=r \times \cos (k), \\
& y^{\prime}=r \times \sin (k),
\end{aligned}
$$

$$
\begin{aligned}
& z^{\prime}=r \times k, \\
& r=u \times e^{k v},
\end{aligned}
$$

where $r$ is the radius of each turn of the spiral and $k$ is a random number in the range $[0,2 \pi] . u$ and $v$ are constants to define the spiral shape, and $e$ is the base of the natural logarithm. The updated position of search agent is calculated using equations (5)-(9).

$$
\vec{P}_{s}(x)=\left(\vec{D}_{s} \times x^{\prime} \times y^{\prime} \times z^{\prime}\right)+\vec{P}_{b s}(x),
$$

where $\vec{P}_{s}(x)$ saves the best solution and updates the position of other search agents. The pseudocode for the SOA is provided in Algorithm 1.

\section{Whale Optimization Algorithm (WOA)}

Whale optimization algorithm (WOA) is a new metaheuristic algorithm that was proposed by Australian scholar Mirialili and others in 2016. The main inspiration of the algorithm is to simulate the predation behaviors of humpback whale population and update the position of candidate solution through the process of whale population encircling prey, spiral updating position, and finding prey. The first stage of the algorithm is to surround prey and spiral bubble net attack (exploitation phase); the second stage is whales randomly looking for food (exploration phase). The following describes the detailed steps of the WOA.

3.1. Encircling Prey: If $p<0.5$ and $|A|<1$. Whales can identify the location of prey and surround them. Assuming that the optimal position in the current population is prey, other whale individuals surround the optimal individuals. Use formula (10) to update the position:

$$
\begin{aligned}
\vec{D} & =\left|\vec{C} \cdot \vec{X}^{*}(t)-\vec{X}(t)\right|, \\
\vec{X}(t+1) & =\vec{X}^{*}(t)-\vec{A} \cdot \vec{D},
\end{aligned}
$$

where $t$ is the current iteration number; $\vec{X}^{*}$ is the prey position; and $\vec{A}$ and $\vec{C}$ are coefficient vectors, and $\vec{A}$ and $\vec{C}$ can be expressed as

$$
\begin{aligned}
& \vec{A}=2 \vec{a} \cdot \vec{r}_{1}-\vec{a}, \\
& \vec{C}=2 \cdot \vec{r}_{2},
\end{aligned}
$$

where $\vec{r}_{1}$ and $\vec{r}_{2}$ are the random vectors between $[0,1]$; is the convergence factor, which linearly decreases from 2 to 0 as the number of iterations increases; the formula is defined as 
Input: Seagull population $\vec{P}_{s}$

Output: Optimal search agent $\vec{P}_{b s}$

(1) Procedure SOA

(2) Initialize the parameters $A, B$ and Max $_{\text {iteration }}$

(3) Set $f_{c} \longleftarrow 2$

(4) Set $u \longleftarrow 1$

(5) Set $v \longleftarrow 1$

(6) While $\left(x<\operatorname{Max}_{\text {iteration }}\right)$ do

(7) $\vec{P}_{b s} \longleftarrow$ ComputeFitness $\left(\vec{P}_{s}\right) / *$ Calculate fitness values of search agent using ComputeFitness $* / / *$ Migration behavior $* /$

(8) $\quad \mathrm{rd} \longleftarrow \operatorname{Rand}(0,1) / *$ To generate the random number in range $[0,1] * /$

(9) $\quad k \longleftarrow \operatorname{Rand}(0,2 \pi) / *$ To generate the random number in range $[0,2 \pi] * / / *$ Attacking behavior $* /$

(10) $\quad r \longleftarrow u \times e^{k v} / *$ To generate the spiral behavior during migration $* /$

(11) Calculate the distance $\vec{D}_{s}$ using equation (5)

(12) $\quad \underline{P} \longleftarrow x \prime \times y \prime \times z \prime / *$ Compute $x, y, z$ planes using equations (6)-(9) $*$ I

(13) $\quad \vec{P}_{s}(x) \longleftarrow\left(\vec{D}_{s} \times P\right)+\vec{P}_{b s}$

(14) $x \longleftarrow x+1$

(15) end while

(16) return $\vec{P}_{b s}$

(17) end procedure

(1) Procedure ComputeFitness $\left(\vec{P}_{s}\right)$

(2) for $i \longleftarrow 1$ to $n$ do / * Here, $n$ represents the dimension of a given problem $*$ /

(3) $\quad$ FIT $[i] \longleftarrow$ Fitness Function $\left(\vec{P}_{s}(i,:)\right) / *$ Calculate the best fitness of each individual $* /$

(4) end for

(5) $\operatorname{FIT}_{s_{\text {best }}} \longleftarrow$ BEST $\left(\right.$ FIT $\left._{s}\right) / *$ Calculate the best fitness value using BEST function $* /$

(6) return FIT $_{s_{\text {best }}}$

(7) end procedure

(1) Procedure $\mathrm{BEST}\left(\mathrm{FIT}_{s}\right)$

(2) $\mathrm{BEST} \longleftarrow \mathrm{FIT}_{s}[0]$

(3) for $i \longleftarrow 1$ to $n$ do

(4) if $\left(\operatorname{FIT}_{s}[i]<\right.$ Best $)$ then

(5) Best $\longleftarrow$ FIT $_{s}[i]$

(6) end if

(7) end for

(8) return Best / * Return the best fitness value $*$ /

(9) end procedure

Algorithm 1: Pseudocode of the SOA.

(1) Generate the initial population $X_{i}(i=1,2, \ldots, N P)$

(2) Evaluate the fitness for each candidate solutions in $X_{i}$

(3) $X^{*}=$ the best candidate solutions

(4) while the halting criterion is not satisfied do

(5) for $i=1$ to NP do

(6) Update $a, A, C, l$ and $P$;

(7) for 1 to $n$ do

(8) if $P<0.5$ then

(9) if $(|A|<1)$

(10) $\quad D=\left|C \cdot X^{*}-X_{t}\right|$

(11) $\quad X_{i}(j)=\left|X^{*}(j)-A \cdot D\right|$

(12) $\quad$ else if $(|A| \geq 1)$

(13) select a random individual $X_{\text {rand }}$

(14) $D=\left|C \cdot X_{\text {rand }}-X_{t}\right|$

(15) $\quad X_{i}(j)=X_{\text {rand }}(j)-A \cdot D$

(16) end if

(17) else if $P \geq 0.5$ then

(18) $\quad D_{\prime}=\left|X^{*}-X_{t}\right|$

(19) $\quad X_{i}(j)=D \prime \cdot e^{b l} \cdot \cos (2 \pi l)+X *$

(20) end if

(21) end for

(22) Evaluate the fitness for $X_{i}$

(23) end for

(24) end while

Algorithm 2: Pseudocode of the WOA. 


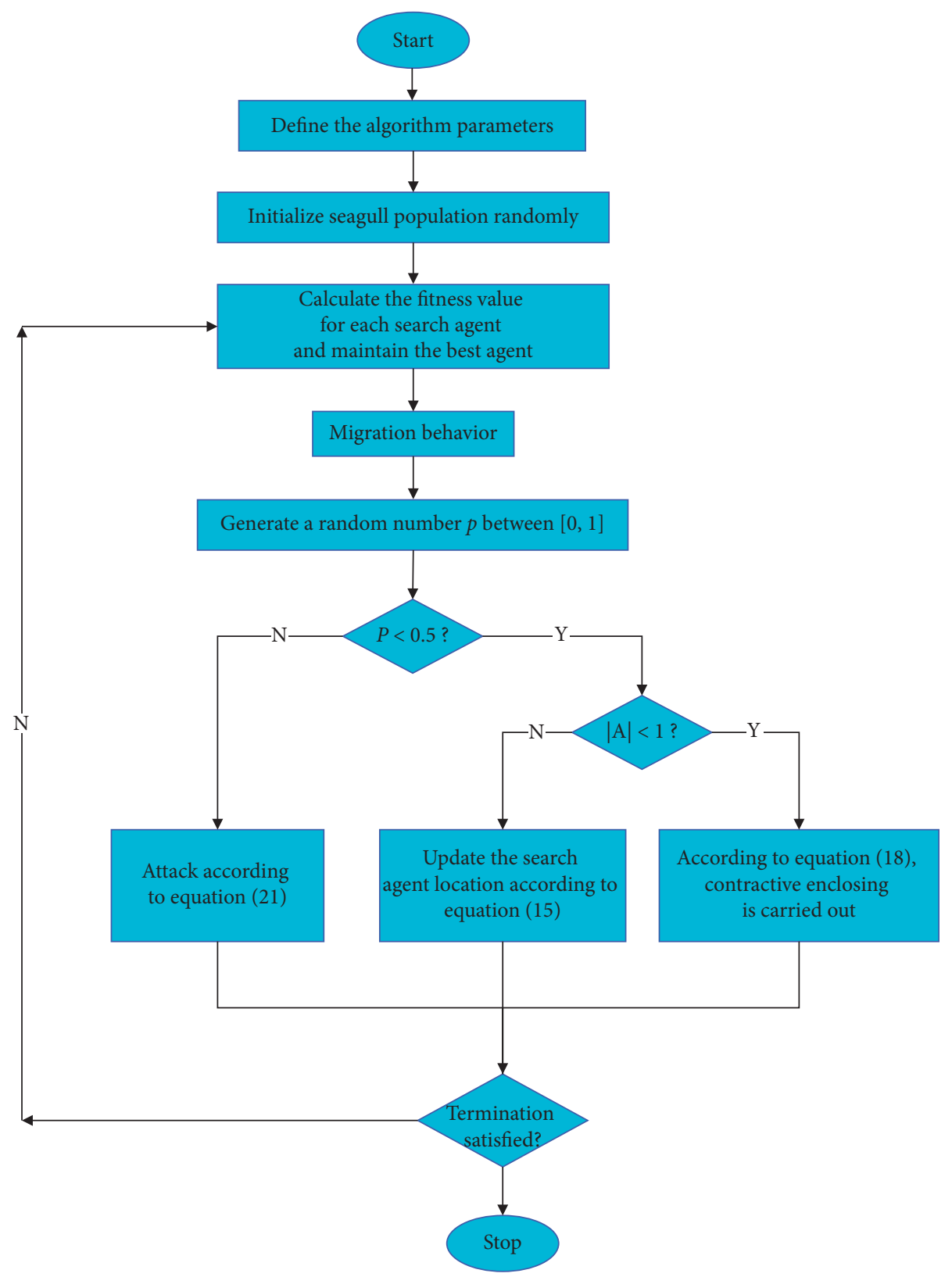

Figure 1: Flow chart of the WSOA.

$$
\vec{a}=2-\frac{2 t}{t_{\max }},
$$

where $t_{\max }$ is the maximum number of iterations.

3.2. Search for Prey: If $p<0.5$ and $|A| \geq 1$. In addition to bubble net predation behavior, whales can also randomly search for food, and the process of searching for food is the exploration stage of the algorithm. That is to say, whale individuals randomly search according to each other's position, and the mathematical model can be expressed as follows:

$$
\begin{aligned}
\vec{D} & =\left|\vec{C} \cdot \vec{X}_{\text {rand }}-\vec{X}\right|, \\
\vec{X}(t+1) & =\vec{X}_{\text {rand }}-\vec{A} \cdot \vec{D},
\end{aligned}
$$

where $\vec{X}_{\text {rand }}$ is the position vector of individual whales randomly selected from the current population.

3.3. Spiral Updating Position: If $p \geq 0.5$. Encircling prey and spiral update position methods are utilized during the exploitation phase of the WOA. The spiral update position formula is as follows: 


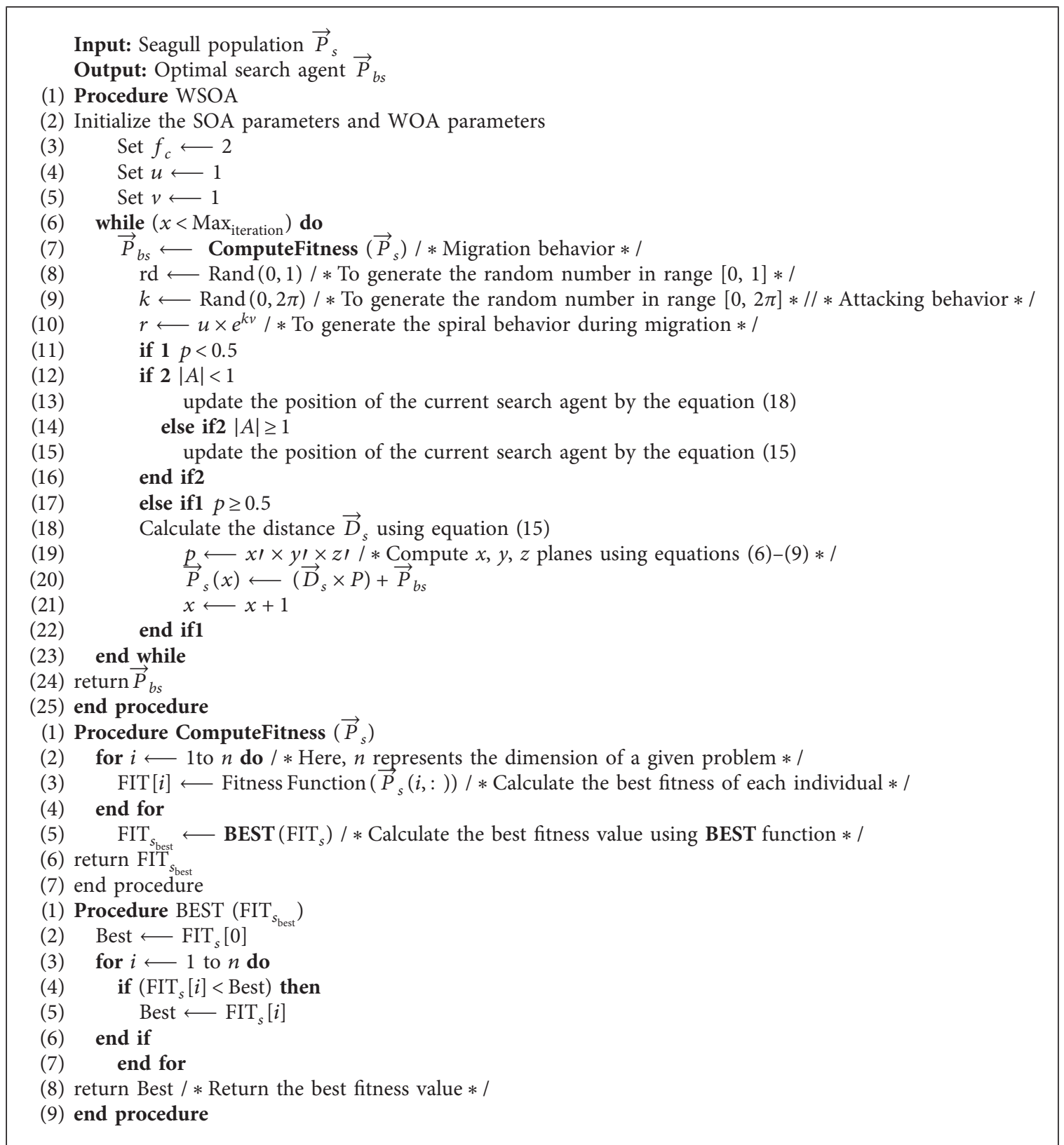

Algorithm 3: Pseudocode of the WSOA.

$$
\vec{X}(t+1)=\vec{D}^{\prime} \cdot e^{b l} \cdot \cos (2 \pi l)+\vec{X}^{*}(t),
$$

where $\vec{D}^{\prime}=\left|\vec{X}_{p}(t)-\vec{X}(t)\right|$, which is the distance between the $i$ th candidate solution and the best solution in the current generation. $b$ is a constant and $l \in[-1,1]$. The specific process of the WOA is reflected in the pseudocode of Algorithm 2.

\section{Hybrid Whale Optimization With Seagull Algorithm (WSOA)}

Many creatures in nature have similarities in the process of foraging. For example, whales have a unique predation behavior in foraging, that is, bubble net foraging behavior.
The WOA designs shrinking encircling mechanism and upward spiral way to attack prey by simulating predation behavior of whales. Seagulls migrate from one place to another to find abundant food when they are looking for food. Once rich prey is found, they will attack prey in a spiral shape in the air. This paper proposes a hybrid whale optimization with seagull algorithm (WSOA) for global optimization problems, which mainly combines the shrinking encircling mechanism of the WOA with the spiral attack behaviors of the SOA, thus greatly improving the local searching and global searching ability of the algorithm. Levy flight is a mechanism of random walking behaviors, which can properly control local search. However, seagull optimization algorithm will converge prematurely. Therefore, this paper also considers the levy flight strategy to be 
TABLE 1: Parameter setting value of algorithm.

\begin{tabular}{|c|c|c|}
\hline Algorithm & Parameter & Value \\
\hline \multirow{2}{*}{ WSOA } & Control parameter $(\mathrm{A})$ & {$[2,0]$} \\
\hline & $f_{c}$ & 2 \\
\hline \multirow{2}{*}{ SOA } & Control parameter (A) & {$[2,0]$} \\
\hline & $f_{c}$ & 2 \\
\hline WOA & A & Decreased from 2 to 0 \\
\hline \multirow{2}{*}{ PSO } & Inertia coefficient & 0.75 \\
\hline & Cognitive and social coeff. & 1.8 and 2 \\
\hline \multirow[t]{2}{*}{ GWO } & Control parameter $(\vec{a})$ & {$[2,0]$} \\
\hline & Number of initial populations $\left(N_{0}\right)$ & 5 \\
\hline \multirow{3}{*}{ IWO } & Maximum number of plant populations & 10 \\
\hline & Maximum number of seeds & 2 \\
\hline & Minimum number of seeds & 0 \\
\hline \multirow{3}{*}{ MFO } & Convergence constant & {$[-1,-2]$} \\
\hline & Logarithmic spiral & 0.75 \\
\hline & Number of onlookers & $50 \%$ of the swarm \\
\hline \multirow{3}{*}{$\mathrm{ABC}$} & Number of employed bees & $50 \%$ of the swarm \\
\hline & Swarm size & 20 \\
\hline & Number of scouts & 1 \\
\hline
\end{tabular}

TABLE 2: Unimodal function.

\begin{tabular}{|c|c|c|c|}
\hline Benchmark test functions & Dim & Range & $f_{\min }$ \\
\hline$f_{01}=\sum_{i=1}^{n} x_{i}^{2}$ & 30 & {$[-100,100]$} & 0 \\
\hline$f_{02}(x)=\sum_{i=1}^{n}\left|x_{i}\right|+\prod_{i=1}^{n}\left|x_{i}\right|$ & 30 & {$[-10,10]$} & 0 \\
\hline$f_{03}(x)=\sum_{i=1}^{n}\left(\sum_{j=1}^{i} x_{j}\right)^{2}$ & 30 & {$[-100,100]$} & 0 \\
\hline$f_{04}(x)=\max _{i}\left\{\left|x_{i}\right|, 1 \leq i \leq D\right\}$ & 30 & {$[-100,100]$} & 0 \\
\hline$f_{05}(x)=\sum_{i=1}^{D-1}\left[100\left(x_{i+1}-x_{i}^{2}\right)^{2}+\left(x_{i}-1\right)^{2}\right]$ & 30 & {$[-30,30]$} & 0 \\
\hline$f_{06}(x)=\sum_{i=1}^{n}\left(\left|x_{i}+0.5\right|\right)^{2}$ & 30 & {$[-100,100]$} & 0 \\
\hline$f_{07}(x)=\sum_{i=1}^{n} x_{i}^{4}+\operatorname{random}(0,1)$ & 30 & {$[-1.28,1.28]$} & 0 \\
\hline
\end{tabular}

introduced into the contraction surrounding mechanism of the WOA and the local searching stage of the SOA, which improves the exploitation ability and avoids the premature convergence of the algorithm. The update formula of contraction surrounding mechanism of the WOA with levy flight strategy is as follows:

$$
\begin{aligned}
\vec{D}_{s} & =\operatorname{levy}(d) \times\left(\operatorname{levy}(d) \times \vec{P}_{b s}(x)-\vec{P}_{s}(x)\right), \\
\vec{P}_{s}(x+1) & =\vec{P}_{b s}(x)-\vec{A} \times \vec{D}_{s},
\end{aligned}
$$

where $d$ is the dimension of the current position vector, $x$ is the current number of iterations, and levy flight [29] can be expressed as

$$
\operatorname{levy}(x)=0.01 \times \frac{r_{1} \times \sigma}{\left|r_{2}\right|^{(1 / \beta)}}
$$

where $r_{1}$ and $r_{2}$ are random numbers in $[0,1], \beta$ is the constant, and $\sigma$ can be expressed as

$$
\sigma=\left(\frac{\Gamma(1+\beta) \times \sin (\pi \beta / 2)}{\Gamma(1+\beta / 2) \times \beta \times 2^{(\beta-1 / 2)}}\right)^{(1 / \beta)},
$$

where $\Gamma(x)=(x-1)$ !
The improvement of spiral attack method of the SOA is as follows:

$$
\begin{aligned}
\vec{D}_{s} & =\vec{C}_{s}+B \times \operatorname{levy}(d) \times\left(\vec{P}_{b s}(x)-\vec{P}_{s}(x)\right), \\
\vec{P}_{s}(x) & =\left(\vec{D}_{s} \times x^{\prime} \times y^{\prime} \times z^{\prime}\right)+\vec{P}_{b s}(x),
\end{aligned}
$$

where $\vec{C}_{s}$ represents the location of the search agent that does not collide with other search agents, $\vec{P}_{s}$ represents the current location of the search agent, represents the distance between the search agent and best-fit search agent, and represents the best-fit search agent. Figure 1 describes the flow chart of WSOA. The pseudocode of the WSOA is shown in Algorithm 3.

\section{Experimental Results and Analysis}

In this section, 25 benchmark functions are used to test the performance of the proposed WSOA. This section is divided into five parts. In Section 5.1, the benchmark function is introduced in detail. Performance analysis of the proposed WSOA and comparison algorithm is shown in Section 5.2. In Section 5.3, statistical test of the algorithm is provided. Section 5.4 analyzes the results. Finally, the proposed WSOA is applied to solve engineering optimization problems in Section 5.5. 


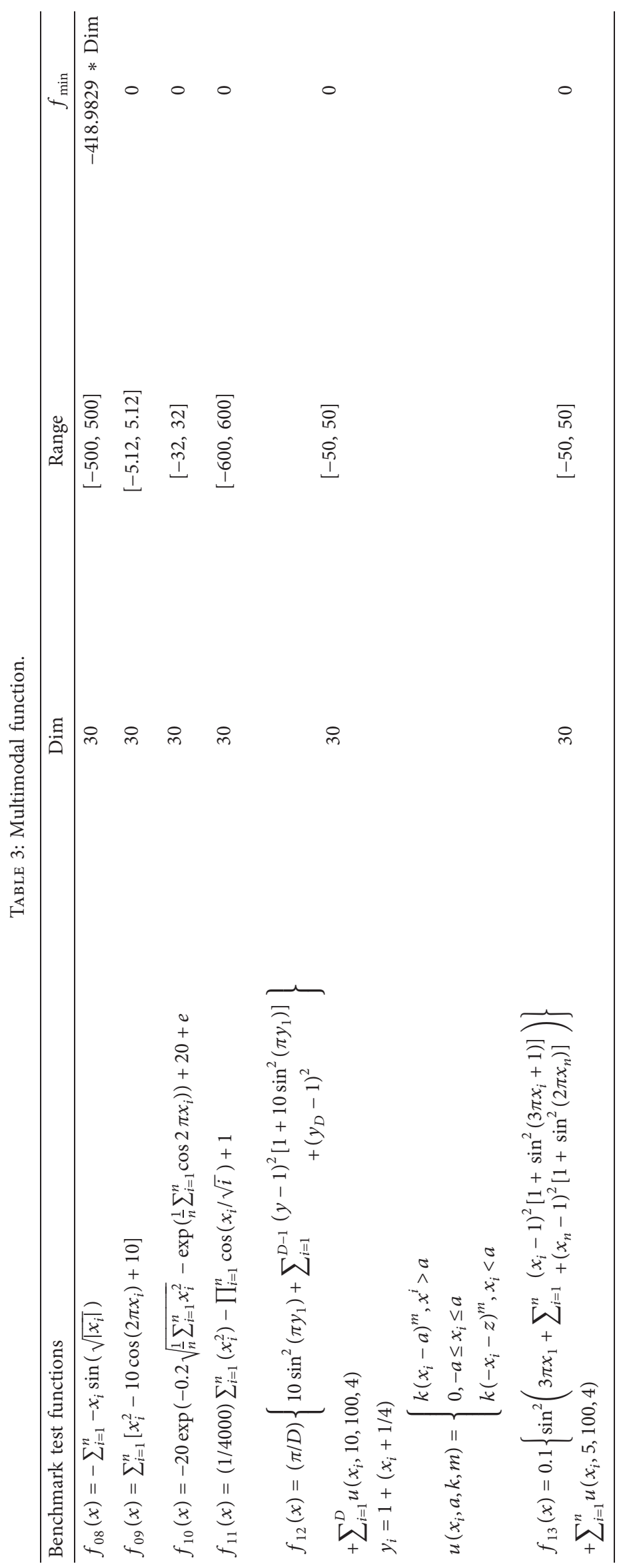


TABLE 4: Fixed-dimensional multimodal function.

\begin{tabular}{|c|c|c|c|}
\hline Benchmark test functions & Dim & Range & $f_{\min }$ \\
\hline$f_{14}(x)=\left(1 / 500+\sum_{j=1}^{25} 1 / j+\sum_{i=1}^{2}\left(x_{i}-a_{i j}\right)^{6}\right)^{-1}$ & 2 & {$[-65,65]$} & 1 \\
\hline$f_{15}(x)=\sum_{i=1}^{11}\left[a_{i}-\left(x_{1}\left(b_{i}^{2}+b_{i} x_{2}\right) / b_{i}^{2}+b_{i} x_{3}+x_{4}\right)\right]^{2}$ & 4 & {$[-5,5]$} & 0.0003075 \\
\hline$f_{16}(x)=4 x_{1}^{2}-2.1 x_{1}^{4}+(1 / 3) x_{1}^{6}+x_{1} x_{2}-4 x_{2}^{2}+4 x_{2}^{4}$ & 2 & {$[-5,5]$} & -1.0316285 \\
\hline$f_{17}(x)=-\left(1+\cos \left(12 \sqrt{x_{1}^{2}+x_{2}^{2}}\right) / 0.5\left(x_{1}^{2}+x_{2}^{2}\right)+2\right)$ & 2 & {$[-5.12,5.12]$} & -1 \\
\hline $\begin{array}{l}f_{18}(x)=\left[1+\left(x_{1}+x_{2}+1\right)^{2}\left(19-14 x_{1}+3 x_{1}^{2}-14 x_{2}+6 x_{1} x_{2}+3 x_{2}^{2}\right)\right] \\
\times\left[30+\left(2 x_{1}-3 x_{2}\right)^{2}\left(18-32 x_{1}+12 x_{1}^{2}+48 x_{2}-36 x_{1} x_{2}+27 x_{2}^{2}\right)\right]\end{array}$ & 2 & {$[-5,5]$} & 3 \\
\hline$f_{19}(x)=-\sum_{i=1}^{4} c_{i} \exp \left(-\sum_{j=1}^{6} a_{i j}\left(x_{j}-p_{i j}\right)^{2}\right)$ & 6 & {$[0,1]$} & -3.32 \\
\hline$f_{20}(x)=-\sum_{i=1}^{5}\left[\left(x-a_{i}\right)\left(x-a_{i}\right)^{T}+c_{i}\right]^{-1}$ & 4 & {$[0,10]$} & -10.1532 \\
\hline$f_{21}(x)=-\sum_{i=1}^{7}\left[\left(x-a_{i}\right)\left(x-a_{i}\right)^{T}+c_{i}\right]^{-1}$ & 4 & {$[0,10]$} & -10.4029 \\
\hline$f_{22}(x)=-\sum_{i=1}^{10}\left[\left(x-a_{i}\right)\left(x-a_{i}\right)^{T}+c_{i}\right]^{-1}$ & 4 & {$[0,10]$} & -10.5364 \\
\hline$f_{23}(x)=-\cos \left(x_{1}\right) \cos \left(x_{2}\right) \exp \left(-\left(x_{1}-\pi\right)^{2}-\left(x_{2}-\pi\right)^{2}\right)$ & 2 & {$[-100,100]$} & -1 \\
\hline$f_{24}(x)=0.5+\left(\sin ^{2}\left(\sqrt{x_{1}^{2}+x_{2}^{2}}\right)-0.5 /\left(1+0.001\left(x_{1}^{2}+x_{2}^{2}\right)\right)^{2}\right)$ & 2 & {$[-100,100]$} & -1 \\
\hline$f_{25}(x)=\sum_{i=1}^{n} x_{i} \sin \left(x_{i}\right)+0.1 x_{i}$ & 10 & {$[-10,10]$} & 0 \\
\hline
\end{tabular}

TABle 5: Test results of unimodal test function.

\begin{tabular}{|c|c|c|c|c|c|}
\hline Functions & Algorithms & Mean & Best & Worst & Std. \\
\hline \multirow{8}{*}{$f_{1}$} & WSOA & $8.47 E-219$ & $6.06 E-241$ & $2.40 E-217$ & 0 \\
\hline & SOA & $3.82 E-28$ & $2.90 E-31$ & $3.66 E-27$ & $7.17 E-28$ \\
\hline & WOA & $2.71 E-147$ & $5.60 E-173$ & $7.03 E-146$ & $1.29 E-146$ \\
\hline & PSO & $7.37 E-14$ & $1.20 E-16$ & $1.09 E-12$ & $2.07 E-13$ \\
\hline & GWO & $9.65 E-59$ & $3.72 E-61$ & $1.01 E-57$ & $2.06 E-58$ \\
\hline & IWO & 1259.1673 & 14.367 & 4578.0546 & 974.9639 \\
\hline & MFO & 1666.6669 & $1.06 E-05$ & 10000.0001 & 3790.4901 \\
\hline & $\mathrm{ABC}$ & 6.9723 & 0.0075248 & 60.4577 & 15.8377 \\
\hline \multirow{8}{*}{$f_{2}$} & WSOA & $2.36 E-112$ & $1.68 E-129$ & $4.52 E-111$ & $9.30 E-112$ \\
\hline & SOA & $5.31 E-18$ & $3.89 E-19$ & $3.05 E-17$ & $6.93 E-18$ \\
\hline & WOA & $6.02 E-101$ & $5.56 E-116$ & $1.81 E-99$ & $3.30 E-100$ \\
\hline & PSO & 0.0063353 & $6.60 E-08$ & 0.18941 & 0.034578 \\
\hline & GWO & $6.95 E-35$ & $8.01 E-36$ & $2.59 E-34$ & $6.01 E-35$ \\
\hline & IWO & 0.032918 & 0.026614 & 0.043399 & 0.0038925 \\
\hline & MFO & 34.6673 & 0.00053366 & 100 & 20.4653 \\
\hline & $\mathrm{ABC}$ & 0.49868 & 0.029053 & 1.8489 & 0.45169 \\
\hline \multirow{8}{*}{$f_{3}$} & WSOA & $2.46 E-153$ & $2.54 E-203$ & $7.37 E-152$ & $1.34 E-152$ \\
\hline & SOA & $3.27 E-13$ & $5.75 E-18$ & $6.58 E-12$ & $1.24 E-12$ \\
\hline & WOA & 18928.8069 & 3503.1755 & 38099.6547 & 9210.6917 \\
\hline & PSO & 110.9042 & 8.0146 & 427.2507 & 108.2086 \\
\hline & GWO & $1.60 E-15$ & $4.72 E-20$ & $3.17 E-14$ & $5.84 E-15$ \\
\hline & IWO & 8484.0385 & 4740.4266 & 13760.1827 & 2323.4503 \\
\hline & MFO & 19544.2017 & 465.4813 & 55008.1336 & 15635.4144 \\
\hline & $\mathrm{ABC}$ & 43922.9306 & 30253.8188 & 65581.9318 & 8548.9204 \\
\hline \multirow{8}{*}{$f_{4}$} & WSOA & $7.93 E-98$ & $1.37 E-119$ & $2.20 E-96$ & $4.01 E-97$ \\
\hline & SOA & $6.71 E-08$ & $8.42 E-11$ & $1.24 E-06$ & $2.27 E-07$ \\
\hline & WOA & 22.1714 & 0.048485 & 76.5243 & 20.0257 \\
\hline & PSO & 3.6386 & 1.0381 & 8.1432 & 1.7835 \\
\hline & GWO & $1.44 E-14$ & $1.18 E-15$ & $1.14 E-13$ & $2.30 E-14$ \\
\hline & IWO & 38.0993 & 28.1075 & 49.4369 & 5.754 \\
\hline & MFO & 69.0251 & 52.7901 & 85.3707 & 8.6404 \\
\hline & $\mathrm{ABC}$ & 85.9882 & 79.2378 & 92.8272 & 3.3293 \\
\hline \multirow{8}{*}{$f_{5}$} & WSOA & 4.7201 & 0.0069459 & 27.7934 & 10.3352 \\
\hline & SOA & 28.1858 & 27.1821 & 28.8496 & 0.52153 \\
\hline & WOA & 27.2119 & 26.3476 & 28.7456 & 0.63476 \\
\hline & PSO & 136.1758 & 7.1025 & 2495.505 & 450.714 \\
\hline & GWO & 26.8744 & 25.9231 & 28.54 & 0.64642 \\
\hline & IWO & 118.1505 & 22.5334 & 1030.8263 & 233.6927 \\
\hline & MFO & 18555.9331 & 6.81 & 90085.3516 & 36367.0326 \\
\hline & $\mathrm{ABC}$ & 3413.6593 & 512.2443 & 18158.4492 & 3832.0029 \\
\hline
\end{tabular}


TABle 5: Continued.

\begin{tabular}{|c|c|c|c|c|c|}
\hline Functions & Algorithms & Mean & Best & Worst & Std. \\
\hline \multirow{8}{*}{$f_{6}$} & WSOA & 0.002881 & 0.00070095 & 0.0070674 & 0.001618 \\
\hline & SOA & 3.1986 & 2.6531 & 3.9467 & 0.36716 \\
\hline & WOA & 0.10824 & 0.008272 & 0.3296 & 0.1094 \\
\hline & PSO & $9.21 E-14$ & $3.62 E-16$ & $7.66 E-13$ & $1.73 E-13$ \\
\hline & GWO & 0.6821 & $1.01 E-05$ & 1.5062 & 0.37642 \\
\hline & IWO & 1148.6679 & $4.65 E-05$ & 3399.7094 & 988.8429 \\
\hline & MFO & 2336.7252 & $2.08 E-05$ & 10100.2501 & 4308.358 \\
\hline & $\mathrm{ABC}$ & 6.7192 & 0.0052413 & 97.6377 & 18.5232 \\
\hline \multirow{8}{*}{$f_{7}$} & WSOA & 0.00016715 & $3.22 E-06$ & 0.0011171 & 0.00020972 \\
\hline & SOA & 0.0010557 & $2.15 E-05$ & 0.0039959 & 0.00088306 \\
\hline & WOA & 0.0021791 & $1.31 E-05$ & 0.0092708 & 0.0023497 \\
\hline & PSO & 0.58978 & 0.081789 & 1.1513 & 0.29928 \\
\hline & GWO & 0.00074907 & 0.00013394 & 0.0019163 & 0.0072772 \\
\hline & IWO & 0.021927 & 0.010026 & 0.036071 & 0.0072772 \\
\hline & MFO & 4.1674 & 0.056477 & 24.2912 & 6.6287 \\
\hline & $\mathrm{ABC}$ & 0.38307 & 0.23624 & 0.59655 & 0.095506 \\
\hline
\end{tabular}

TABLE 6: Test results of multimodal test function.

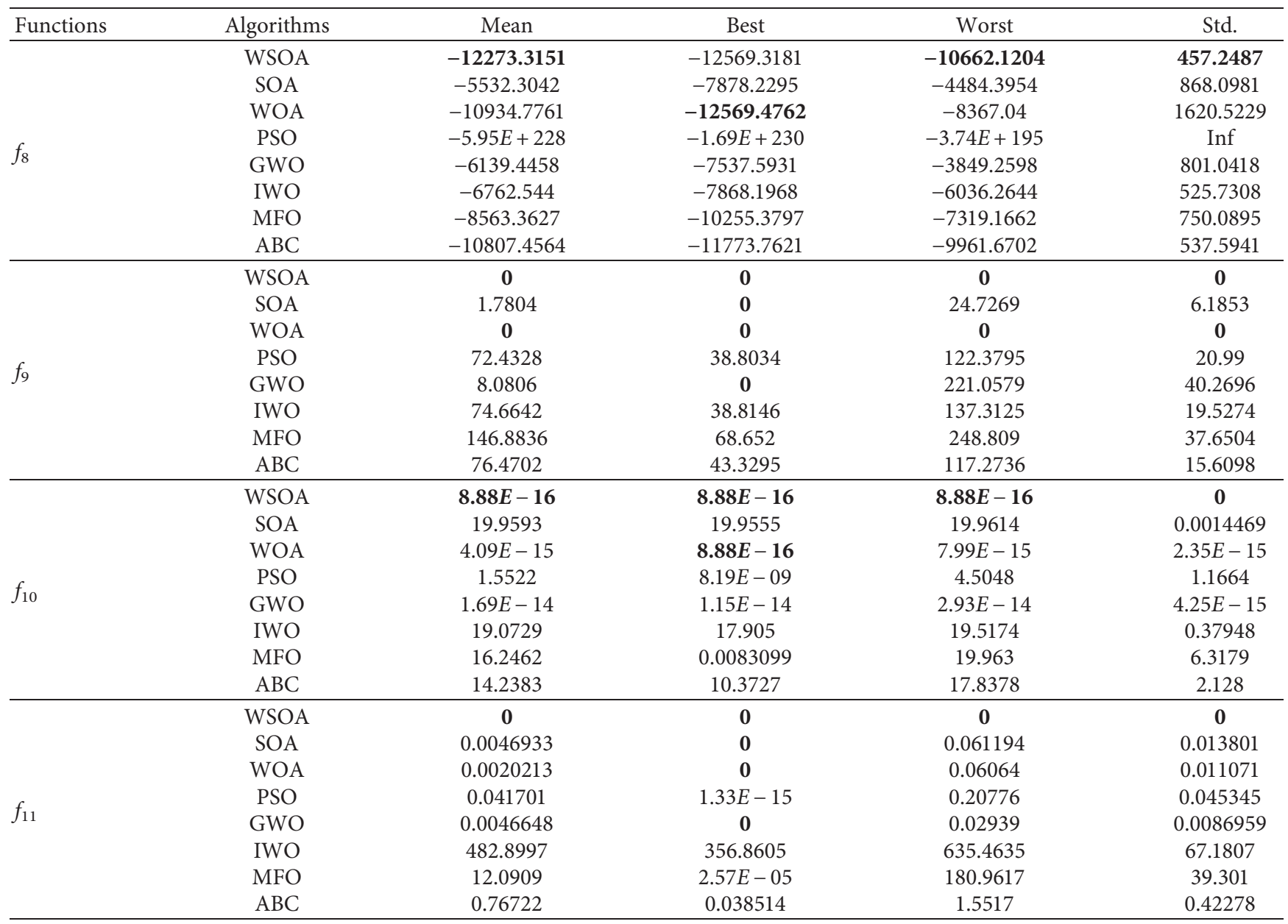


TABle 6: Continued.

\begin{tabular}{|c|c|c|c|c|c|}
\hline Functions & Algorithms & Mean & Best & Worst & Std. \\
\hline \multirow{8}{*}{$f_{12}$} & WSOA & 0.00032222 & $3.56 E-05$ & 0.00098051 & 0.00023705 \\
\hline & SOA & 0.30919 & 0.13453 & 0.82893 & 0.12059 \\
\hline & WOA & 0.0066089 & 0.0006208 & 0.02561 & 0.0069502 \\
\hline & PSO & 0.34246 & $2.16 E-13$ & 1.5421 & 0.44148 \\
\hline & GWO & 0.036269 & 0.01308 & 0.098627 & 0.019866 \\
\hline & IWO & 20.185 & 10.9406 & 31.9883 & 5.3077 \\
\hline & MFO & 8533335.445 & $6.93 E-06$ & 256000015.7 & 46738994.14 \\
\hline & $\mathrm{ABC}$ & 0.46742 & 0.020219 & 1.1086 & 0.33933 \\
\hline \multirow{8}{*}{$f_{13}$} & WSOA & 0.0093616 & 0.0010055 & 0.051019 & 0.01256 \\
\hline & SOA & 1.9594 & 1.4542 & 2.4358 & 0.23239 \\
\hline & WOA & 0.24625 & 0.026198 & 0.5863 & 0.15422 \\
\hline & PSO & 0.38429 & $1.15 E-16$ & 3.5975 & 1.0906 \\
\hline & GWO & 0.48834 & $7.24 E-05$ & 1.1269 & 0.22795 \\
\hline & IWO & 0.00037624 & $6.61 E-06$ & 0.011 & 0.0020065 \\
\hline & MFO & 13668758.95 & 0.00012905 & 410062760.4 & 74866874.57 \\
\hline & $\mathrm{ABC}$ & 1.6428 & 0.16393 & 5.2668 & 1.2614 \\
\hline
\end{tabular}

TABLE 7: Test results of fixed-dimensional multimodal test function.

\begin{tabular}{|c|c|c|c|c|c|}
\hline Functions & Algorithms & Mean & Best & Worst & Std. \\
\hline \multirow{8}{*}{$f_{14}$} & WSOA & 0.998 & 0.998 & 0.998 & $1.85 E-13$ \\
\hline & SOA & 2.3207 & 0.998 & 2.9821 & 1.1455 \\
\hline & WOA & 2.3207 & 0.998 & 2.9821 & 1.1455 \\
\hline & PSO & 0.998 & 0.998 & 0.9980 & 0 \\
\hline & GWO & 0.998 & 0.998 & 0.998 & $1.16 E-10$ \\
\hline & IWO & 11.6069 & 5.9288 & 21.9884 & 9.0039 \\
\hline & $\mathrm{MFO}$ & 1.3293 & 0.998 & 1.992 & 0.5739 \\
\hline & $\mathrm{ABC}$ & 0.998 & 0.998 & 0.998 & $1.17 E-13$ \\
\hline \multirow{8}{*}{$f_{15}$} & WSOA & 0.00056838 & 0.00030764 & 0.0015736 & 0.00036169 \\
\hline & SOA & 0.0012309 & 0.00066377 & 0.0013874 & 0.0001195 \\
\hline & WOA & 0.0010588 & 0.00030758 & 0.013197 & 0.0023246 \\
\hline & PSO & 0.00070654 & 0.00030749 & 0.0010683 & 0.00029363 \\
\hline & GWO & 0.0024196 & 0.00030749 & 0.020363 & 0.0060904 \\
\hline & IWO & 0.0013891 & 0.0003075 & 0.020363 & 0.0036025 \\
\hline & MFO & 0.0022639 & 0.0003704 & 0.020363 & 0.0049334 \\
\hline & $\mathrm{ABC}$ & 0.0012599 & 0.00062549 & 0.0019125 & 0.00031881 \\
\hline \multirow{8}{*}{$f_{16}$} & WSOA & -1.0316 & -1.0316 & -1.0316 & $1.07 E-08$ \\
\hline & SOA & -1.0316 & -1.0316 & -1.0316 & $4.41 E-07$ \\
\hline & WOA & -1.0316 & -1.0316 & -1.0316 & $2.29 E-10$ \\
\hline & PSO & -1.0316 & -1.0316 & -1.0316 & $6.71 E-16$ \\
\hline & GWO & -1.0316 & -1.0316 & -1.0316 & $4.23 E-09$ \\
\hline & IWO & -1.0316 & -1.0316 & -1.0316 & $4.51 E-09$ \\
\hline & MFO & -1.0316 & -1.0316 & -1.0316 & $6.78 E-16$ \\
\hline & $\mathrm{ABC}$ & -1.0316 & -1.0316 & -1.0316 & $1.16 E-15$ \\
\hline \multirow{8}{*}{$f_{17}$} & WSOA & -1 & -1 & -1 & 0 \\
\hline & SOA & -1 & -1 & -1 & $\mathbf{0}$ \\
\hline & WOA & -0.97237 & -1 & -0.93625 & 0.032133 \\
\hline & PSO & -0.99787 & -1 & -0.93625 & 0.01164 \\
\hline & GWO & -1 & -1 & -1 & 0 \\
\hline & IWO & -1 & -1 & -1 & $3.60 E-08$ \\
\hline & MFO & -0.9745 & -1 & -0.93625 & 0.031767 \\
\hline & $\mathrm{ABC}$ & -0.99878 & -1 & -0.99583 & 0.0011721 \\
\hline
\end{tabular}


TABle 7: Continued.

\begin{tabular}{|c|c|c|c|c|c|}
\hline Functions & Algorithms & Mean & Best & Worst & Std. \\
\hline \multirow{8}{*}{$f_{18}$} & WSOA & 3 & 3 & 3 & $7.28 E-05$ \\
\hline & SOA & 3 & 3 & 3.0001 & $2.19 E-05$ \\
\hline & WOA & 3 & 3 & 3.0002 & $3.51 E-05$ \\
\hline & PSO & 3 & 3 & 3 & $1.37 E-15$ \\
\hline & GWO & 5.7 & 3 & 84 & 14.7885 \\
\hline & IWO & 3 & 3 & 3 & $3.46 E-07$ \\
\hline & MFO & 3 & 3 & 3 & $1.98 E-15$ \\
\hline & $\mathrm{ABC}$ & 3.0016 & 3 & 3.0227 & 0.0043786 \\
\hline \multirow{8}{*}{$f_{19}$} & WSOA & -3.322 & -3.322 & -3.322 & $3.31 E-05$ \\
\hline & SOA & -3.0507 & -3.121 & -3.0156 & 0.060884 \\
\hline & WOA & -3.3218 & -3.3219 & -3.3216 & 0.00015049 \\
\hline & PSO & -3.2824 & -3.322 & -3.2031 & 0.068643 \\
\hline & GWO & -3.2409 & -3.322 & -3.1978 & 0.070281 \\
\hline & IWO & -3.2031 & -3.2031 & -3.2031 & $2.42 E-06$ \\
\hline & MFO & -3.1785 & -3.2031 & -3.1327 & 0.039669 \\
\hline & $\mathrm{ABC}$ & -3.322 & -3.322 & -3.322 & $1.48 E-06$ \\
\hline \multirow{8}{*}{$f_{20}$} & WSOA & -10.1532 & -10.1532 & -10.1531 & $3.45 E-05$ \\
\hline & SOA & -6.8513 & -10.138 & -0.35136 & 5.6292 \\
\hline & WOA & -8.453 & -10.152 & -5.0551 & 2.9427 \\
\hline & PSO & -5.0856 & -5.1008 & -5.0552 & 0.026312 \\
\hline & GWO & -10.1527 & -10.1528 & -10.1524 & 0.0002039 \\
\hline & IWO & -5.138 & -10.1532 & -2.6305 & 4.3432 \\
\hline & MFO & -5.9615 & -10.1532 & -2.6305 & 3.8345 \\
\hline & $\mathrm{ABC}$ & -10.1166 & -10.1489 & -10.0844 & 0.032264 \\
\hline \multirow{8}{*}{$f_{21}$} & WSOA & -10.4029 & -10.4029 & -10.4027 & $5.51 E-05$ \\
\hline & SOA & -5.8125 & -10.3992 & -0.37244 & 4.3645 \\
\hline & WOA & -8.7562 & -10.4029 & -3.7242 & 2.5549 \\
\hline & PSO & -5.9382 & -10.4029 & -2.7519 & 3.3146 \\
\hline & GWO & -10.4025 & -10.4029 & -10.4015 & 0.00032137 \\
\hline & IWO & -6.4519 & -10.4029 & -2.7519 & 3.594 \\
\hline & MFO & -7.7868 & -10.4029 & -2.7519 & 3.3175 \\
\hline & $\mathrm{ABC}$ & -10.3656 & -10.4029 & -10.1998 & 0.058166 \\
\hline \multirow{8}{*}{$f_{22}$} & WSOA & -10.5364 & -10.5364 & -10.5364 & $1.19 E-05$ \\
\hline & SOA & -7.3236 & -10.5326 & -0.94707 & 5.5222 \\
\hline & WOA & -6.4993 & -10.5342 & -3.8352 & 3.5537 \\
\hline & PSO & -10.5364 & -10.5364 & -10.5364 & 0 \\
\hline & GWO & -10.536 & -10.5364 & -10.5357 & 0.00034804 \\
\hline & IWO & -2.6783 & -2.8066 & -2.4217 & 0.22222 \\
\hline & MFO & -6.0691 & -10.5364 & -3.8354 & 3.8688 \\
\hline & $\mathrm{ABC}$ & -10.5247 & -10.5364 & -10.5183 & 0.010085 \\
\hline \multirow{8}{*}{$f_{23}$} & WSOA & -1 & -1 & -1 & $8.23 E-09$ \\
\hline & SOA & -0.99997 & -1 & -0.99993 & $1.92 E-05$ \\
\hline & WOA & -1 & -1 & -1 & $8.15 E-08$ \\
\hline & PSO & -1 & -1 & -1 & o \\
\hline & GWO & -1 & -1 & -1 & $2.81 E-07$ \\
\hline & IWO & -1 & -1 & -1 & $1.29 E-09$ \\
\hline & MFO & -1 & -1 & -1 & 0 \\
\hline & $\mathrm{ABC}$ & -0.99999 & -1 & -0.99984 & $2.90 E-05$ \\
\hline \multirow{8}{*}{$f_{24}$} & WSOA & -1 & -1 & -1 & 0 \\
\hline & SOA & -0.99806 & -1 & -0.99028 & 0.0039528 \\
\hline & WOA & -0.99514 & -1 & -0.99028 & 0.004941 \\
\hline & PSO & -0.99437 & -1 & -0.99028 & 0.004798 \\
\hline & GWO & -0.99644 & -1 & -0.99028 & 0.0047621 \\
\hline & IWO & -0.61609 & -0.99028 & -0.50405 & 0.13302 \\
\hline & MFO & -0.9919 & -1 & -0.99028 & 0.0036828 \\
\hline & $\mathrm{ABC}$ & -0.99159 & -0.99998 & -0.99024 & 0.0027293 \\
\hline
\end{tabular}


TABLE 7: Continued.

\begin{tabular}{lccccc}
\hline Functions & Algorithms & Mean & Best & Worst & Std. \\
\hline & WSOA & $\mathbf{2 . 8 6 E}-\mathbf{9 8}$ & $\mathbf{3 . 2 7 E - 1 3 3}$ & $\mathbf{8 . 5 7 E - 9 7}$ & $\mathbf{1 . 5 6 E - 9 7}$ \\
& SOA & $1.21 E-24$ & $1.43 E-35$ & $3.64 E-23$ & $0.65 E-24$ \\
& WOA & 0.42502 & $5.15 E-120$ & 3.6239 & 0.98594 \\
$f_{25}$ & PSO & $2.98 E-15$ & $2.29 E-28$ & $1.85 E-14$ & $3.89 E-15$ \\
& GWO & 0.00010204 & $8.13 E-68$ & 0.00096615 & 0.00021053 \\
& IWO & 0.026443 & 0.0090162 & 0.066102 & 0.011482 \\
& MFO & 0.14801 & $2.88 E-22$ & 4.4402 & 0.81067 \\
& ABC & 0.048445 & 0.0042035 & 0.17205 & 0.046518 \\
\hline
\end{tabular}

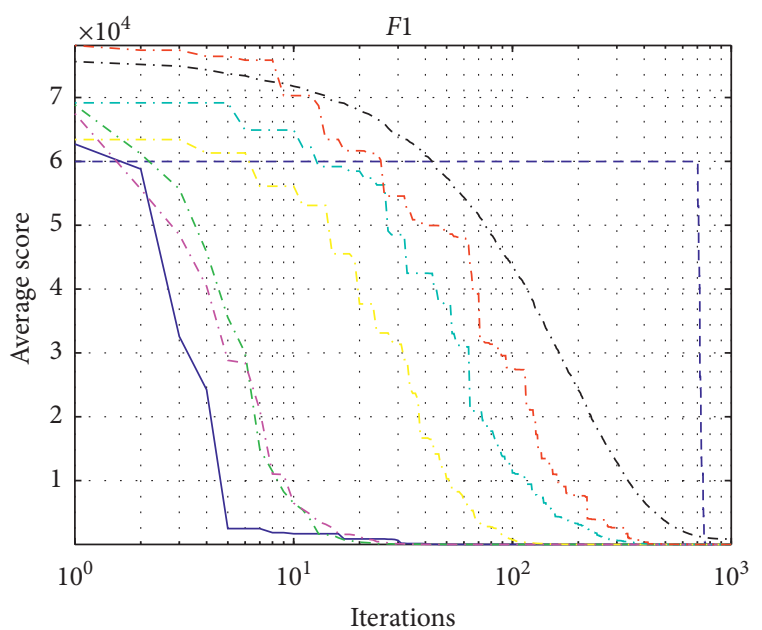

$\begin{array}{ll}\text { - WSOA } & \ldots \text { GWO } \\ -\ldots \text { SOA } & \cdots-\text { IWO } \\ \ldots-\text { WOA } & \cdots-\text { MFO } \\ \ldots-\text { PSO } & \ldots-\text { ABC }\end{array}$

FIgURE 2: Convergence curve of $f_{1}$.

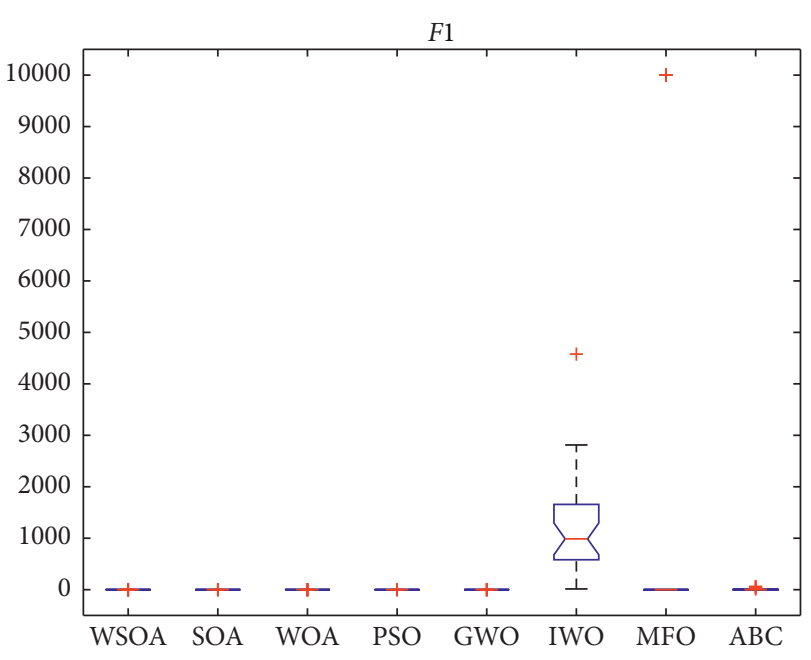

Figure 3: Variance analysis diagram of $f_{1}$.

The experiment is described in detail below. This article selects the seven following algorithms (SOA, WOA, PSO, GWO, IWO, MFO, and ABC) for comparative research. During the experiment, the population size $N$ was set to be

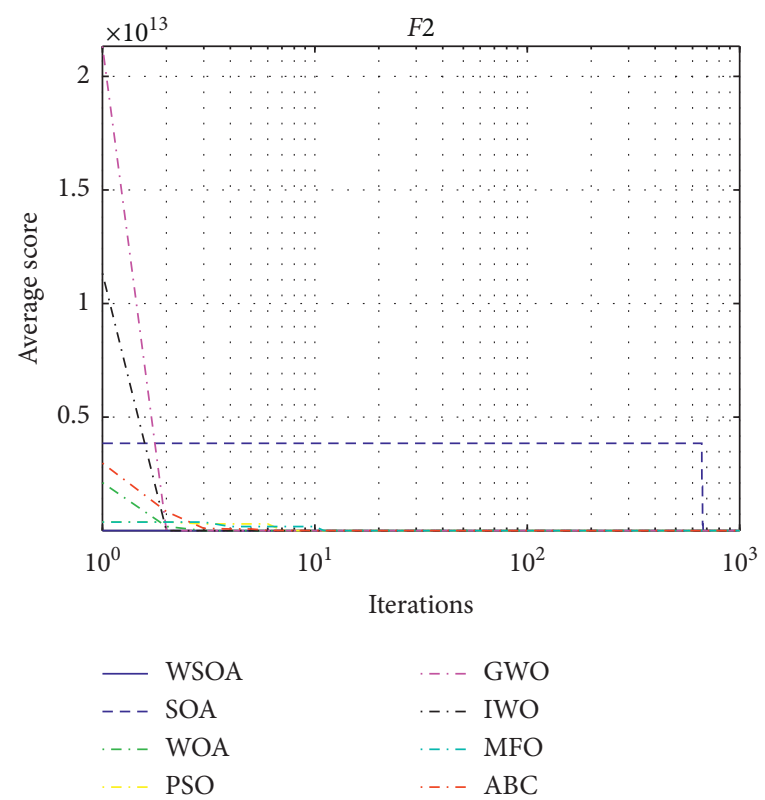

Figure 4: Convergence curve of $f_{2}$.

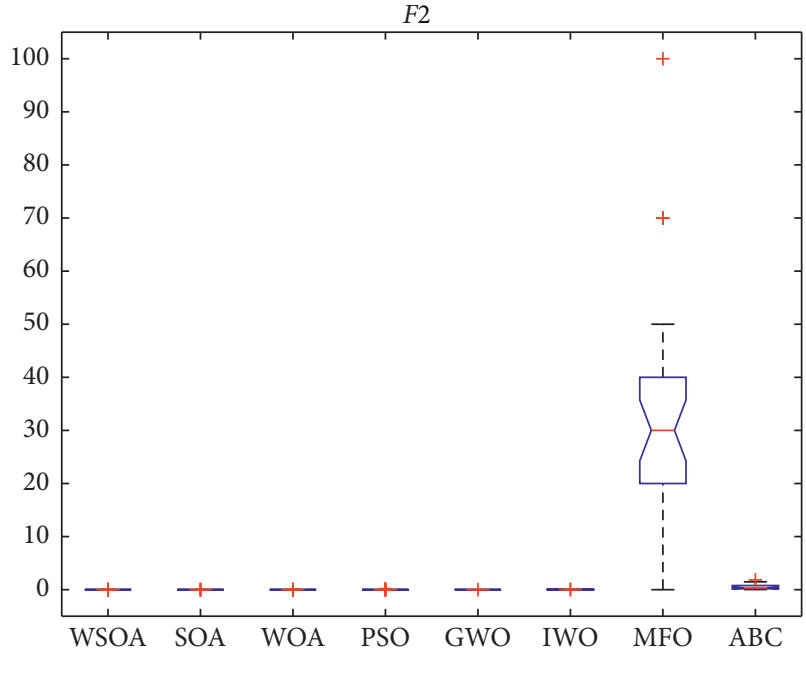

FIgURE 5: Variance analysis diagram of $f_{2}$.

30 , the maximum number of iterations was 1000 , and each benchmark function was run independently for 30 times. All experiments are implemented in MATLAB R2018b and are carried out on a computer with Intel (R) Core (TM) i5- 


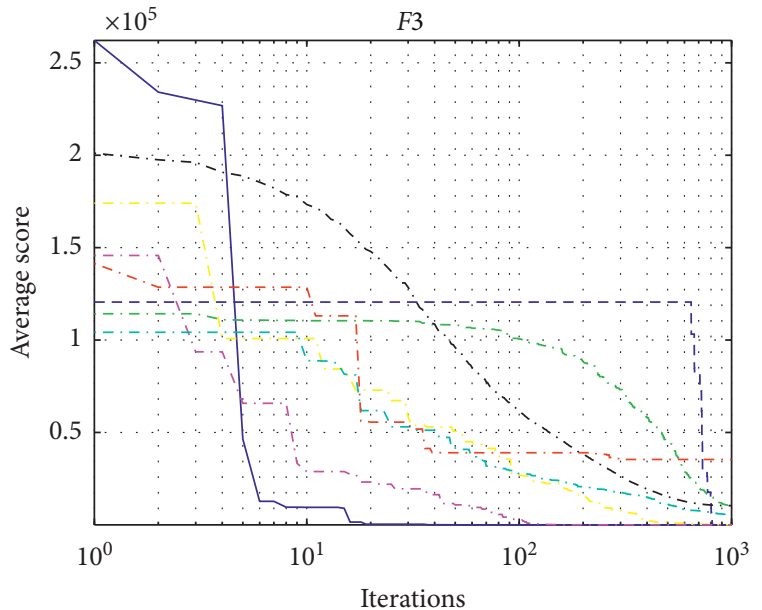

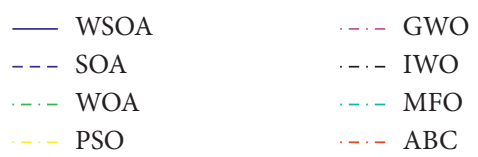

Figure 6: Convergence curve of $f_{3}$.

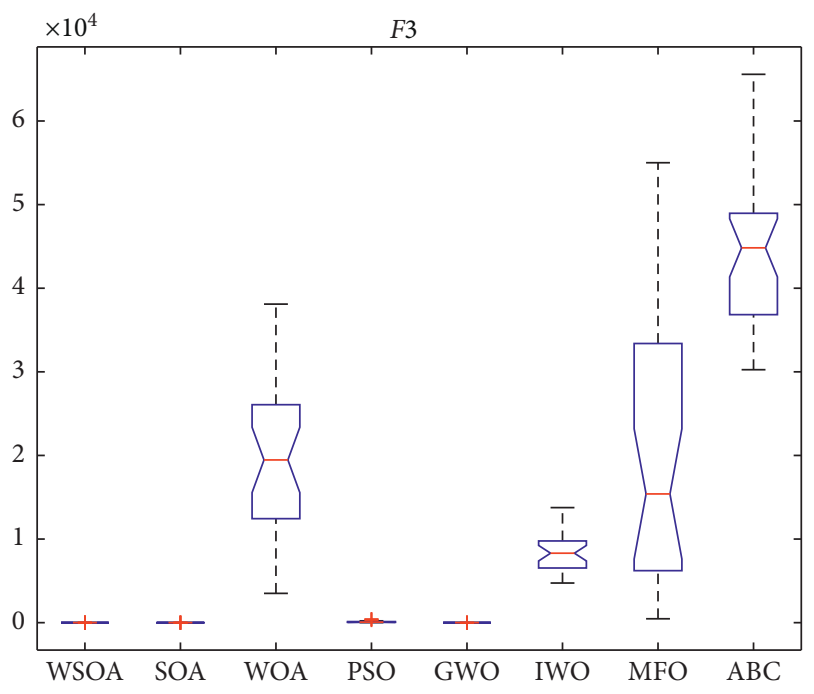

FIgURE 7: Variance analysis diagram of $f_{3}$.

10210U CPU @ $2.11 \mathrm{GHz}$ system type as 64-bit Windows 10 operating system. In addition, in order to make the comparison more convenient, the specific parameters of these seven algorithms can be obtained according to the original paper, as shown in Table 1.

5.1. Benchmark Function. According to the references, we extracted 25 benchmark functions to verify the algorithm [30-32]. These benchmark functions are mainly divided into three categories: unimodal function (Table 2), multimodal function (Table 3), and fixed-dimensional multimodal function (Table 4). Unimodal function has only one optimal solution in a given interval, and these functions can test the exploitation ability of the algorithm. Multimodal function

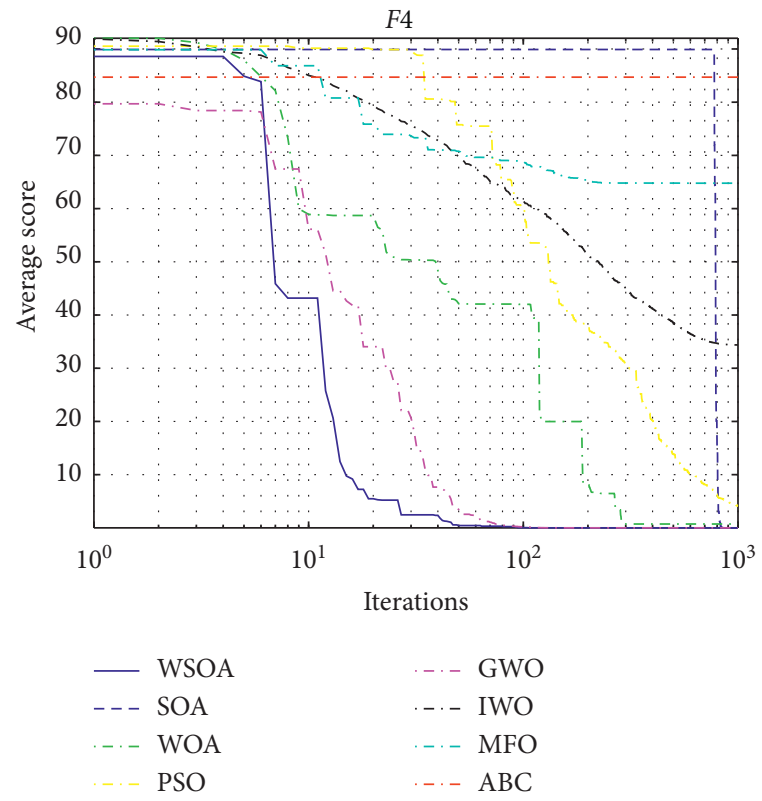

Figure 8: Convergence curve of $f_{4}$.

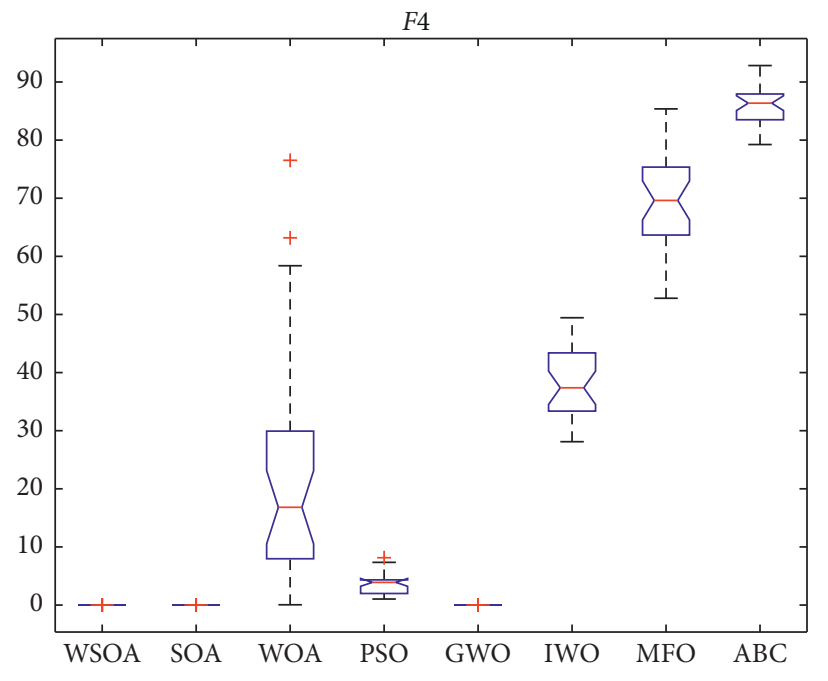

FIgURE 9: Variance analysis diagram of $f_{4}$.

has multiple optimal solutions, among which there is only one global optimal solution, and these functions can test the exploration ability of the algorithm. The detailed information of these 25 benchmark functions is shown in Tables 2-4.

5.2. Performance Comparison and Analysis with Other Algorithms. In this experiment, mean, best, worst, and standard deviation (Std) are used as evaluation indexes. The experimental results of unimodal function are shown in Table 5, those of multimodal function in Table 6, and those of fixed multimodal function in Table 7; bold numbers indicate the optimal solution of comparison algorithm. 

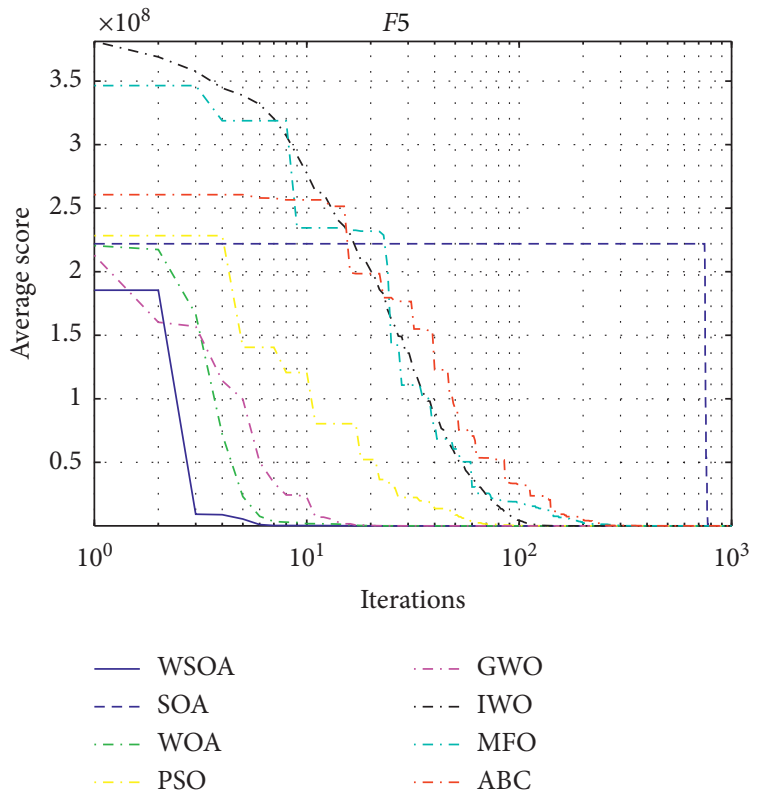

Figure 10: Convergence curve of $f_{5}$.

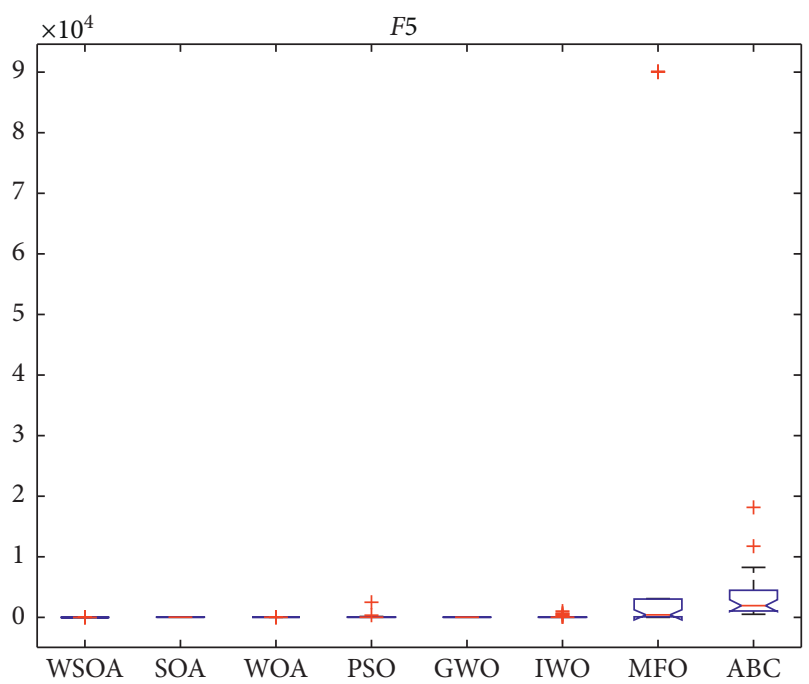

FIGURE 11: Variance analysis diagram of $f_{5}$.

5.2.1. Analysis of Unimodal Function Test Results. Table 5 shows the test results of 8 metaheuristic algorithms in testing unimodal functions $f_{1}-f_{7}$. It can be seen from the table that the WSOA has better performance than other algorithms in most of the tested unimodal functions. Except for function $f_{6}$, which ranks second after PSO, the WSOA ranks first among the other six unimodal test functions $f_{1}-f_{5}$ and $f_{7}$. The variances of functions $f_{1}-f_{4}$ and $f_{7}$ are all in the first place, indicating that the WSOA has better stability. Generally speaking, the WSOA can find the optimal solution faster than other algorithms, which shows that the WSOA has better convergence speed.

Figures 2-15 are the convergence curves and variance analysis diagram of unimodal test functions $f_{1}-f_{7}$. The convergence curve here is drawn according to the average

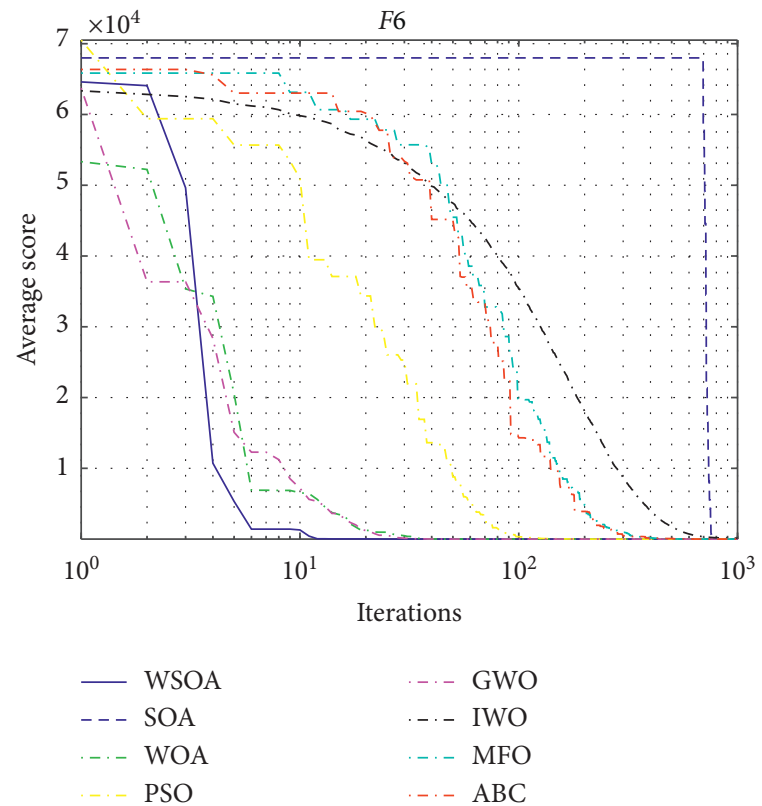

Figure 12: Convergence curve of $f_{6}$.

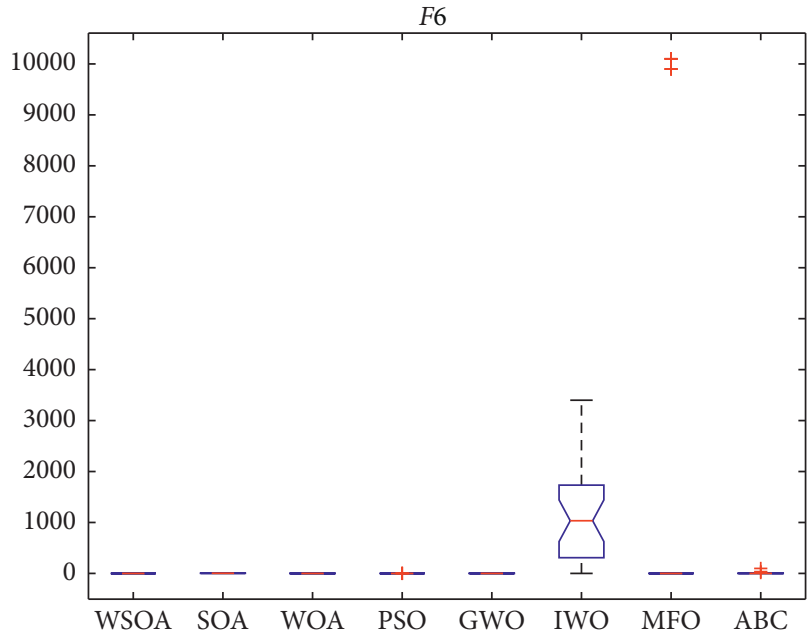

Figure 13: Variance analysis diagram of $f_{6}$.

value of these eight metaheuristic algorithms and is the result of each algorithm running independently for 30 times. It can be seen from the figure that the WSOA converges faster than the other seven algorithms and can find the near-optimal solution faster. It can be seen from Figure 12 that the convergence speed of the WSOA in the early stage is faster than that of the other seven algorithms, and the convergence speed in the later stage is slightly inferior to that of PSO algorithm. The ANOVA chart can directly see the stability of the unimodal function tested by the algorithm; that is to say, the lower the box chart is, the more stable it is. It can be seen from the figure that the variance analysis chart except $f_{5}$ is worse than SOA, WOA, and GWO, ranking fourth. In the ANOVA diagram of test functions $f_{1}-f_{4}, f_{6}$, and $f_{7}$, the variance value of the WSOA is the smallest. Generally speaking, the WSOA has good stability in testing unimodal functions. Through the 

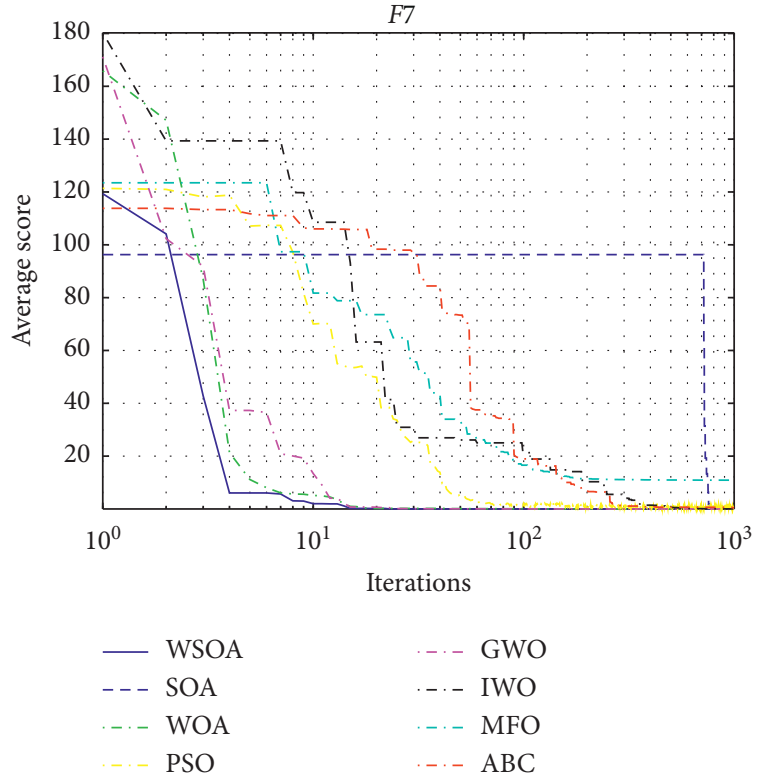

Figure 14: Convergence curve of $f_{7}$.

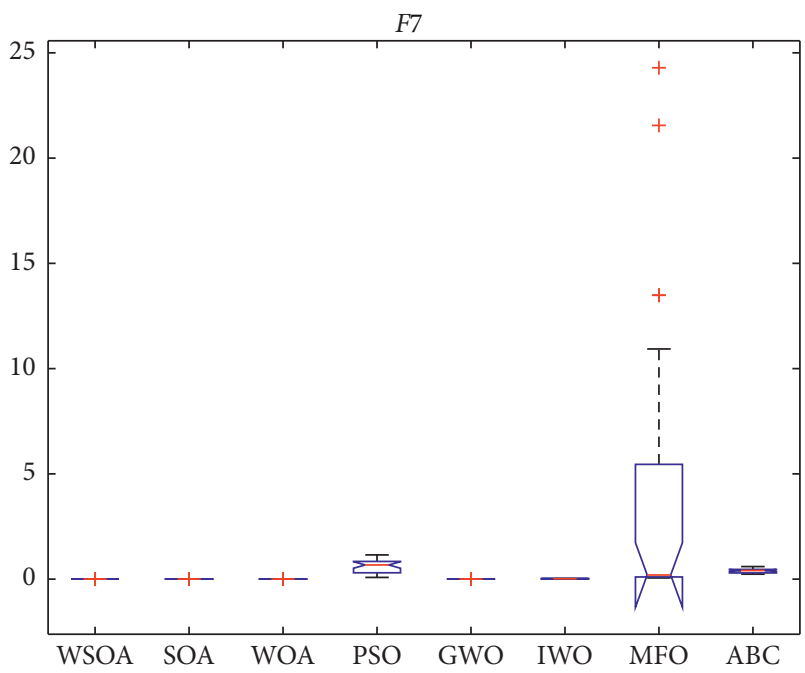

FIgURE 15: Variance analysis diagram of $f_{7}$.

above experiments, it can be proved that the WSOA has strong convergence speed in solving unimodal test functions, which shows that the algorithm has strong global search ability.

5.2.2. Analysis of Multimodal Function Test Results. Table 6 shows the test results of eight metaheuristic algorithms for multimodal test functions $f_{8}-f_{13}$. It can be seen from the table that the WSOA's test results for other multimodal test functions $f_{8}-f_{12}$ are in the first place except that the test results of function $f_{13}$ are slightly inferior to IWO and rank second. Furthermore, the WSOA can accurately find the corresponding theoretical optimal solution in the multimodal test functions $f_{9}$ and $f_{11}$, which fully proves that the WSOA has strong optimization ability. Although the best values of multimodal test functions $f_{8}$ and $f_{12}$ rank

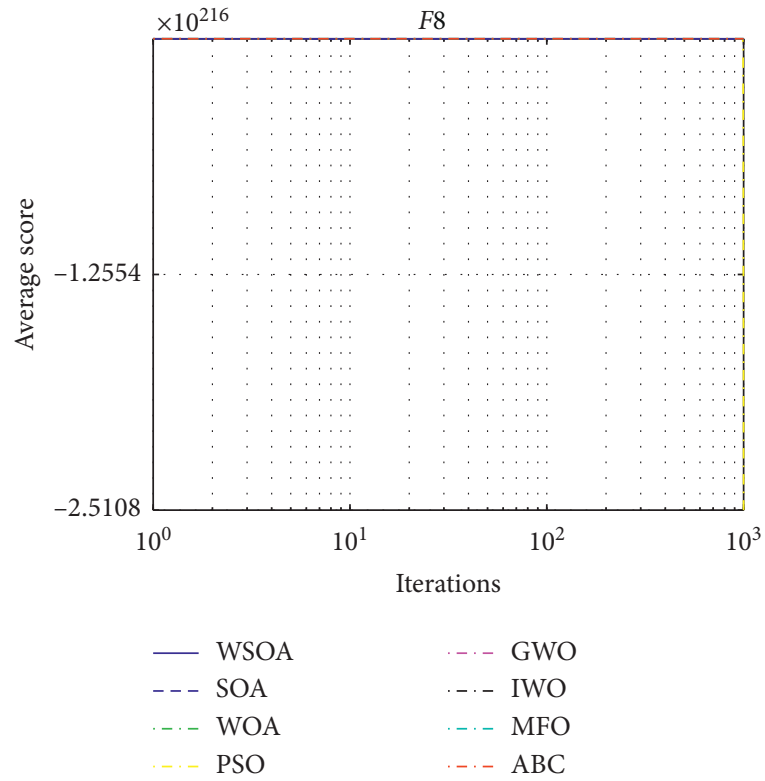

FiguRE 16: Convergence curve of $f_{8}$.

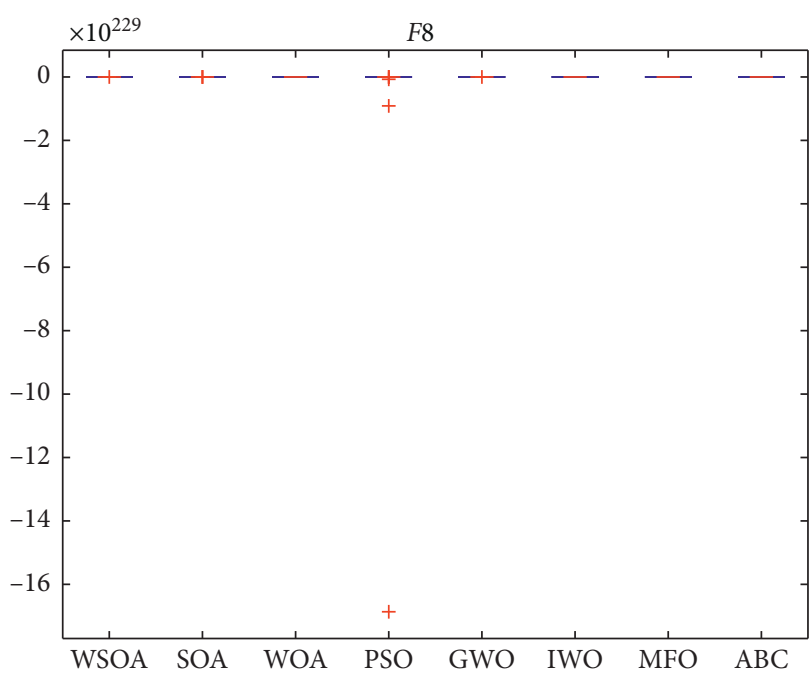

Figure 17: Variance analysis diagram of $f_{8}$.

second, on the whole, the WSOA is superior to the other seven metaheuristic algorithms, and the proposed algorithm has better performance.

Figures 16-27 are the convergence curves and variance analysis graph of the multimodal test functions $f_{8}-f_{13}$. From the convergence graph, it can be seen that the convergence speed and convergence accuracy of the WSOA in multimodal test function are superior to seven other metaheuristic algorithms. In the multimodal test function $f_{13}$ (Figure 26), although the IWO algorithm gets smaller value than the WSOA in the later stage, the convergence speed of the WSOA is obviously better than that of the IWO algorithm in the early stage. On the whole, the WSOA has stronger convergence speed in testing multimodal functions. It can be seen from the analysis of variance that the WSOA can almost get the minimum value in the 


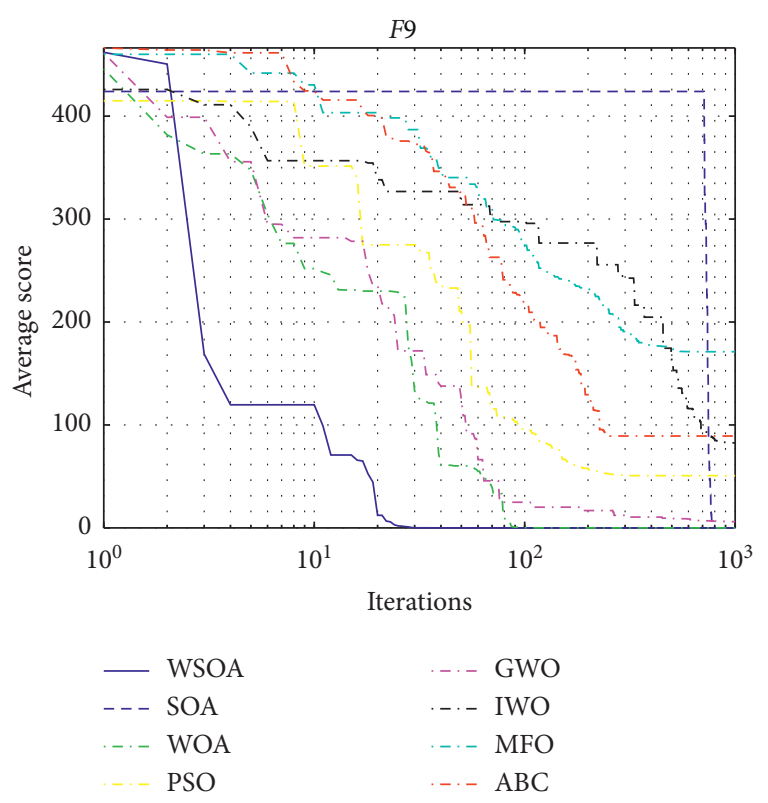

Figure 18: Convergence curve of $f_{9}$.

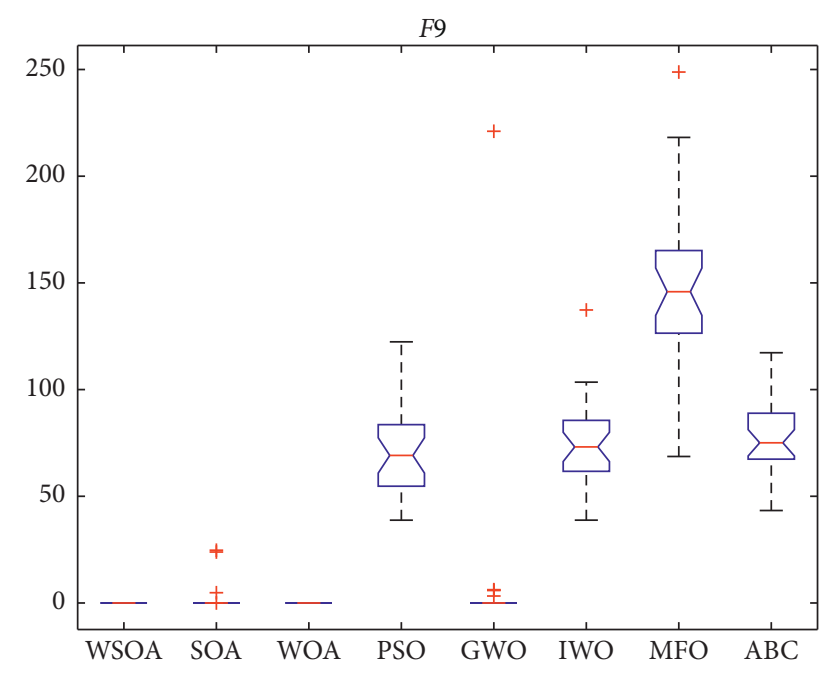

FIGURE 19: Variance analysis diagram of $f_{9}$.

multimodal test function, which shows that it has good stability in the test of multimodal test function.

5.2.3. Analysis of Test Results of Fixed-Dimensional Multimodal Function. The fixed-dimensional multimodal test function is the same as the image of multimodal test function, but their dimensions are different, and the former's dimension is a fixed value. Table 7 shows the test results of eight metaheuristic algorithms on the fixed-dimensional multimodal test functions. It can be seen that the WSOA shows that its average value ranks first in the test of the fixed-dimensional multimodal test functions $f_{14}-f_{25}$ and the best values of the other fixed-dimensional multimodal test functions $f_{14}$ and $f_{16}-f_{25}$ rank first except the best value of function $f_{15}$. Among

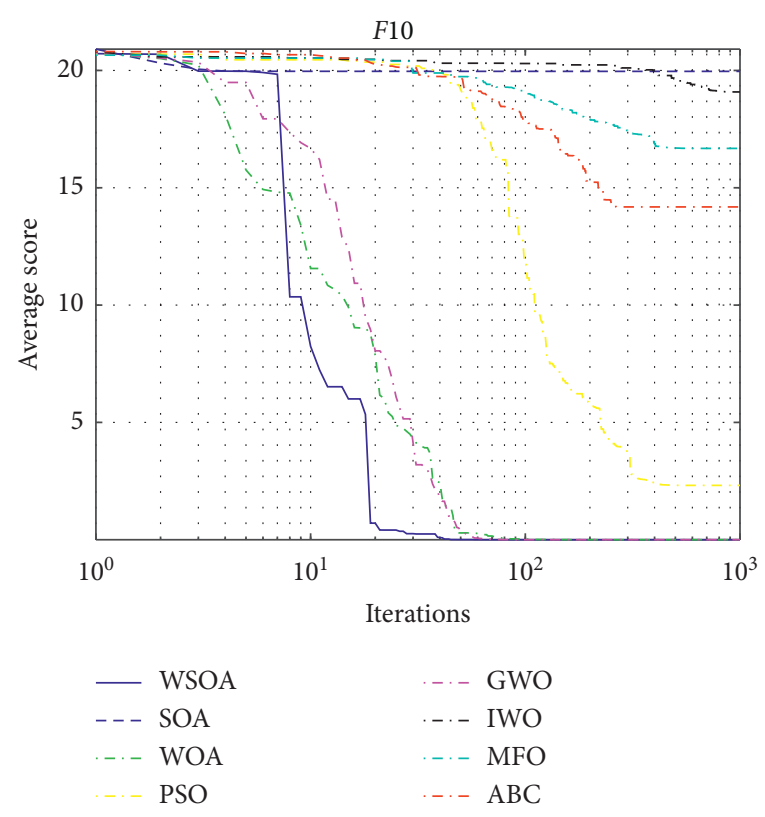

Figure 20: Convergence curve of $f_{10}$.

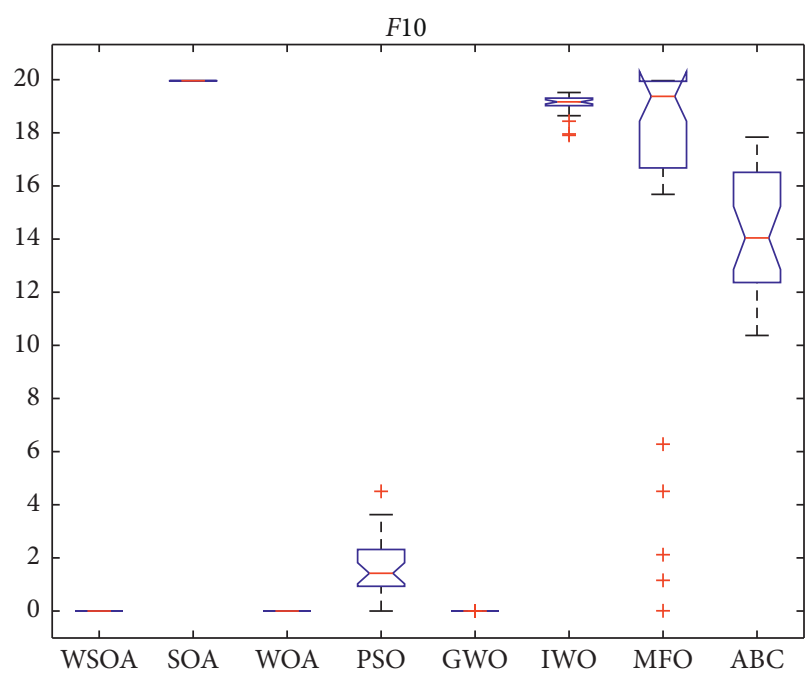

Figure 21: Variance analysis diagram of $f_{10}$.

them, the corresponding theoretical optimum values can be found for functions $f_{16}, f_{17}, f_{18}, f_{20}-f_{24}$, and so forth.

Figures 28-51 are the convergence curves and variance analysis graph of the test results of optimizing the fixed-dimensional multimodal function with 8 metaheuristic algorithms. It can be seen from the convergence graph that the convergence speed of the WSOA algorithm is better than that of seven other algorithms, and the convergence accuracy is also very good, which shows the effectiveness of this algorithm. It can be seen from the analysis of variance that most of the variance values in the fixed-dimensional multimodal functions $f_{14}-f_{25}$ are the smallest, which shows that the algorithm is stable. Generally speaking, the WSOA has better convergence speed than seven other metaheuristic algorithms. 

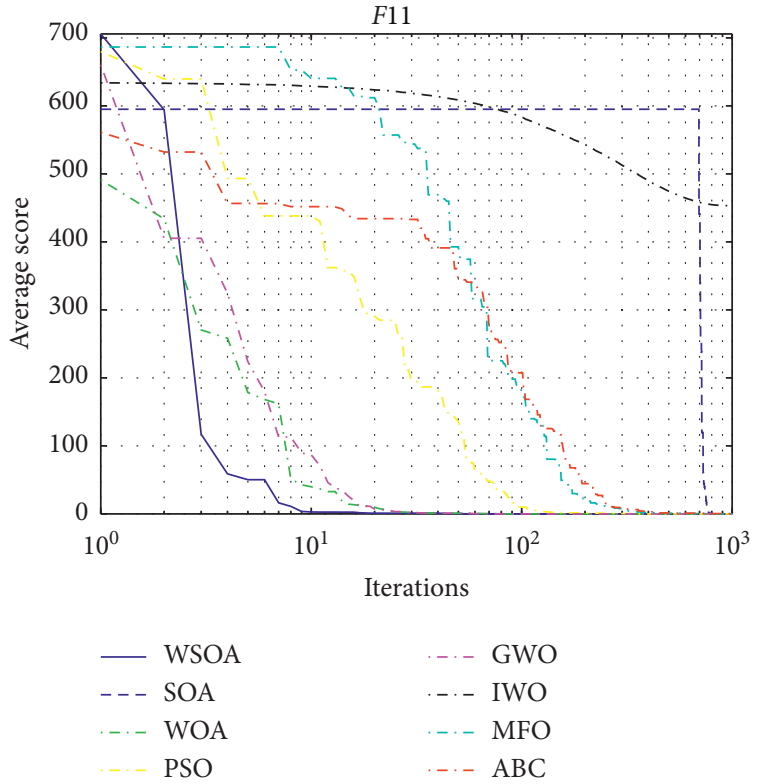

FIGURE 22: Convergence curve of $f_{11}$.

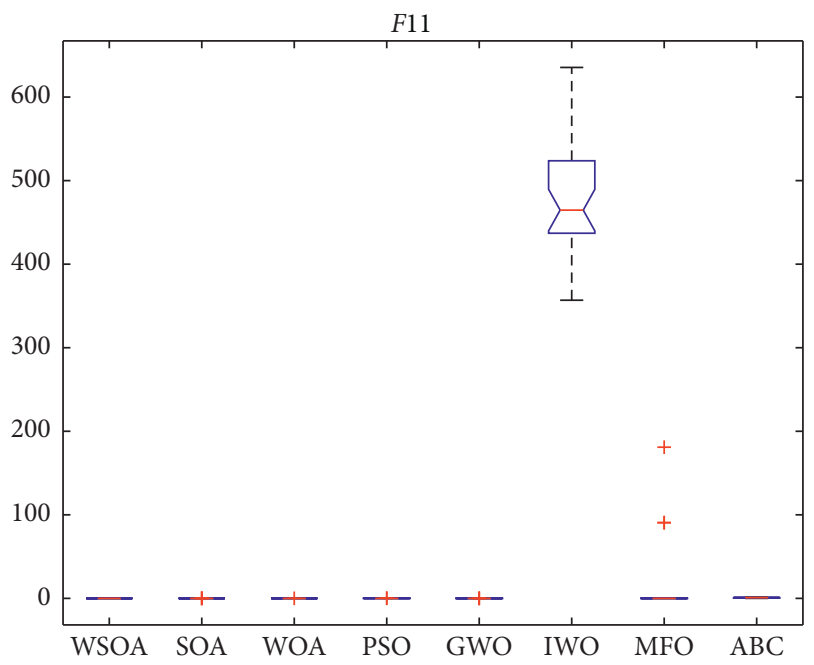

FIgURE 23: Variance analysis diagram of $f_{11}$.

5.3. Statistical Tests. In order to verify the noncontingency of the results, Wilcoxon signed-rank test [33] is carried out in this paper. Wilcoxon signed-rank test compares the performance of the proposed SOA with those of other algorithms, and it is widely used to compare the performances of two optimization algorithms [34-36]. In this experiment, the mean value obtained by running the WSOA algorithm independently for 30 times is compared with the mean values of seven other algorithms, and the Wilcoxon signed-rank test result with significance level of $a=0.05$ is obtained.

Table 8 shows the Wilcoxon signed-rank test results between the WSOA and seven comparison algorithms. In the table, " $\mathrm{H}$ " is marked with " 1 ," which indicates that there is a significant difference between the WSOA and the comparative algorithm, and it has statistical advantage. " $\mathrm{H}$ " marking " 0 " indicates that there is no obvious difference
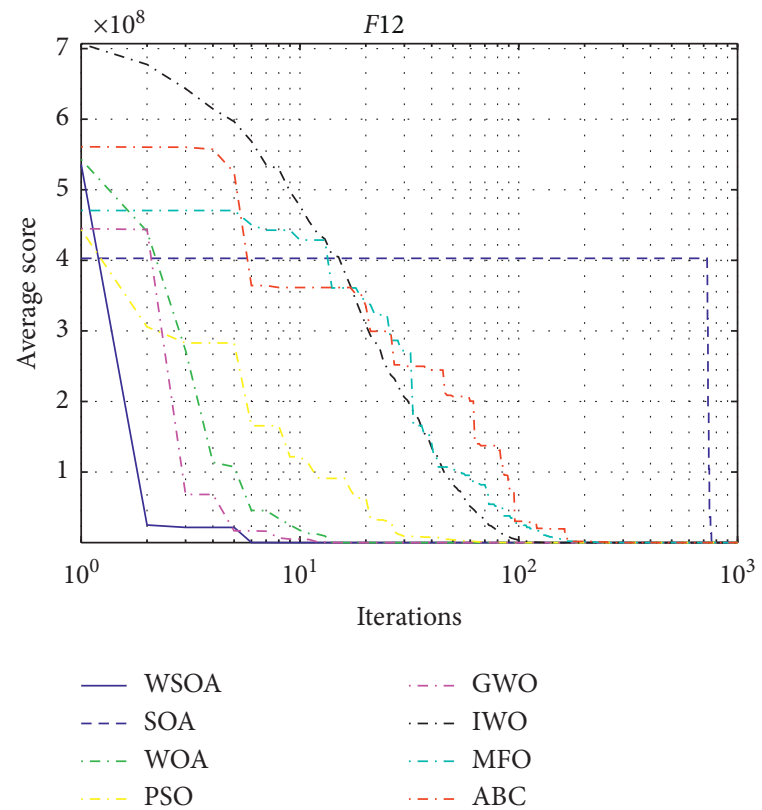

FIgURE 24: Convergence curve of $f_{12}$.

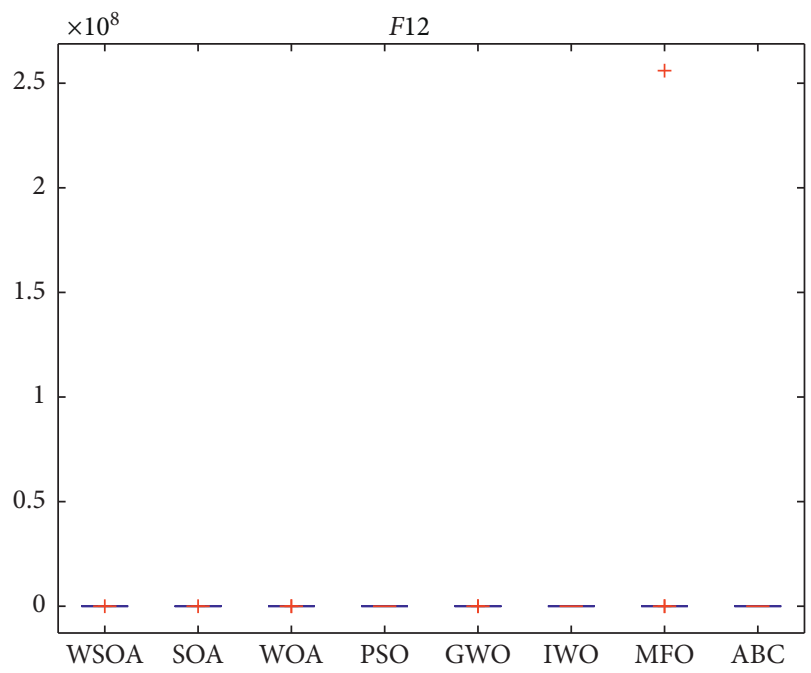

FIgURE 25: Variance analysis diagram of $f_{12}$.

between the WSOA and the comparison algorithm. "S" marking "+" indicates that the performance of the WSOA is better than that of the comparison algorithm. " $\mathrm{S}$ " marking "=" indicates that the performance of the WSOA is the same as that of the comparison algorithm. In addition, the last column of each table under the heading $\mathrm{w} / \mathrm{t} / \mathrm{l}$ represents the win, flat, and lose counts of the WSOA relative to the comparison algorithm. It can be seen from the table that the $\mathrm{ABC}$ algorithm is worse than the WSOA in other test functions except that the test function $f_{23}$ is equal to the WSOA. The WSOA is superior to or equal to SOA, WOA, PSO, GWO, IWO, and MFO in all test functions. More specifically, in the unimodal test functions $f_{1}-f_{7}$, the WSOA is superior to the comparison algorithm, which shows that the performance of the WSOA is superior to the 

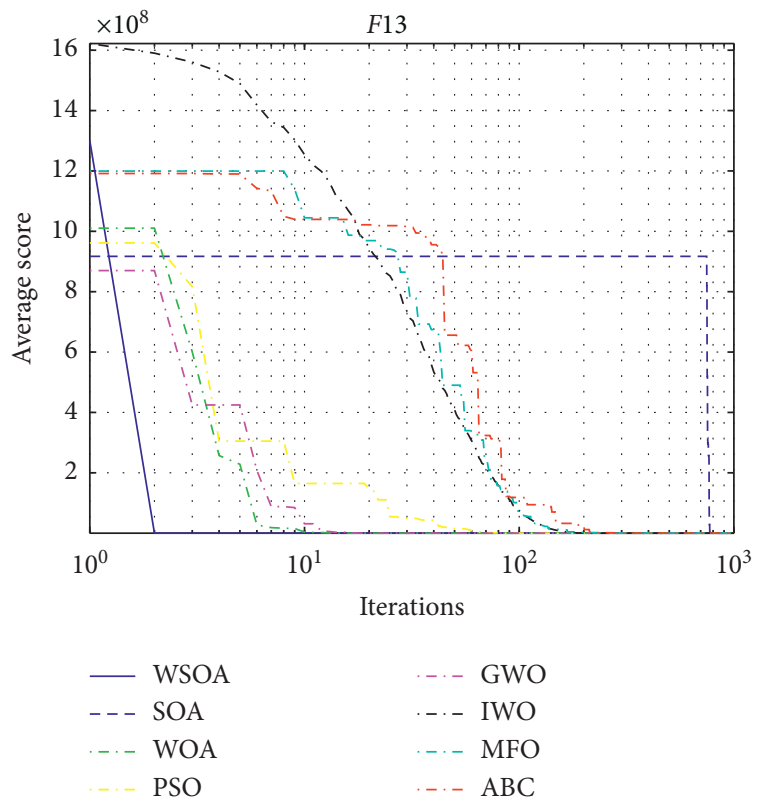

Figure 26: Convergence curve of $f_{13}$.

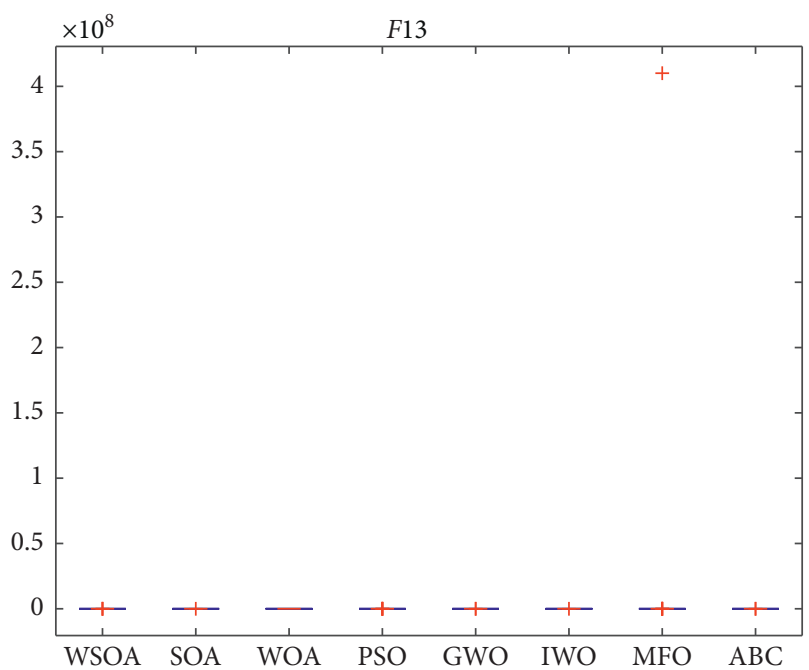

Figure 27: Variance analysis diagram of $f_{13}$.

comparison algorithm, and the exploitation ability of the WSOA is superior to the comparison algorithm. Among the multimodal test functions $f_{8}-f_{13}$, the WSOA is superior to GWO, IWO, MFO, and ABC. Except that $f_{9}$ and $f_{11}$ are equal to the WSOA, SOA and WOA are worse than the WSOA in other test functions. In the fixed multipeak test functions $f_{14}-f_{25}$, the WSOA is superior to or equal to the comparison algorithm. To sum up, the proposed SOA is superior to the comparative algorithm in the stability of global search capability.

In order to further verify the reliability of the algorithm, the Friedman rank test is carried out on the experimental results [37]. Table 9 shows the results of the Friedman rank test. It can be seen from the table that the WSOA ranks first, which shows that the performance of this algorithm is better than that of the comparison algorithm.

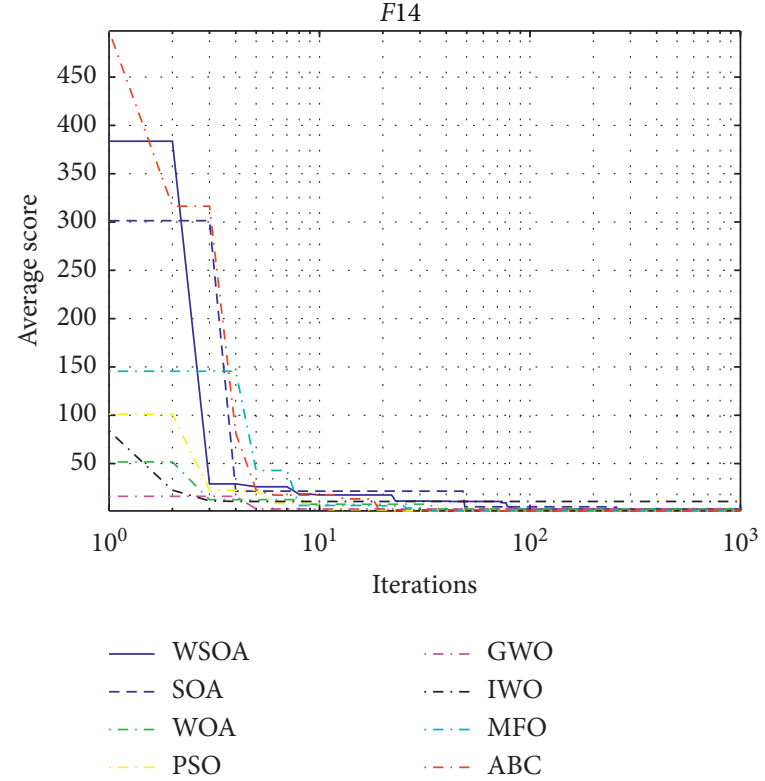

Figure 28: Convergence curve of $f_{14}$.

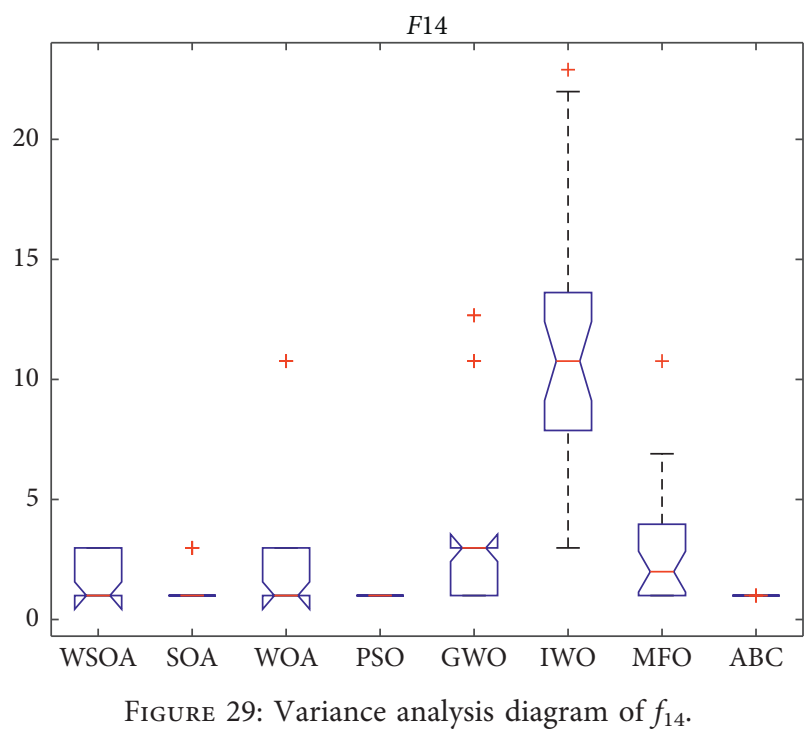

5.4. Result Analysis. Through the above analysis, compared with other comparison algorithms, the proposed WSOA shows superior performance, which shows that the combination of the SOA and the WOA is very successful in solving optimization problems. The reasons for the superior performance can be summarized as follows: Firstly, the local search ability of the WSOA is effectively enhanced by using the fast search ability of contraction and encirclement mechanism of the WOA and spiral attack behavior of the SOA. Secondly, levy flight strategy is introduced, which makes use of the random flight mode characteristics of levy's long-term small-scale search and occasionally large-scale exploration, which is beneficial to the exploration and development of balanced algorithm and avoids premature convergence. Finally, the proposed SOA retains the original migration behavior, which is helpful to improve the search 


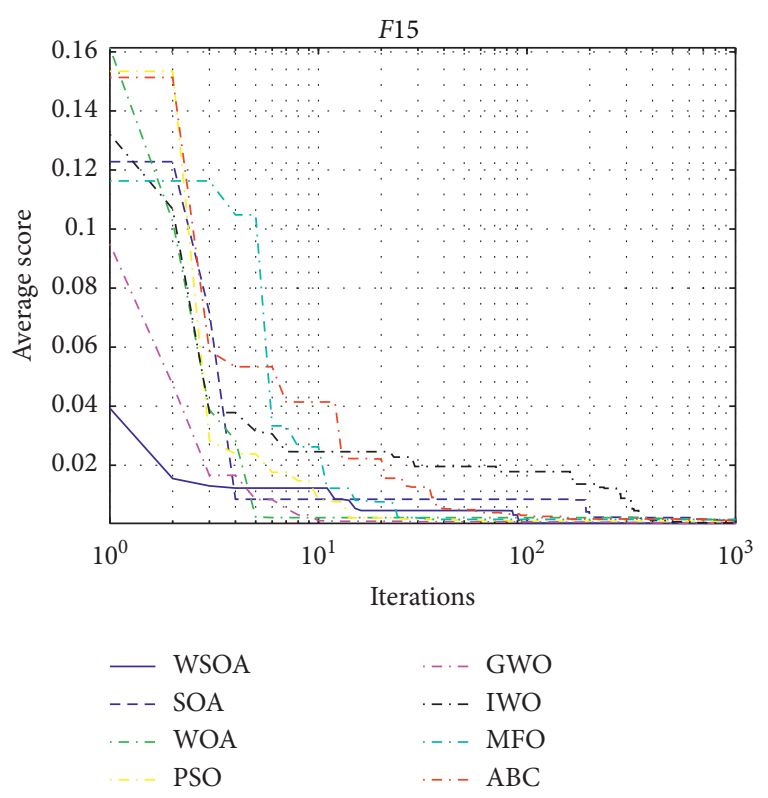

Figure 30: Convergence curve of $f_{15}$.

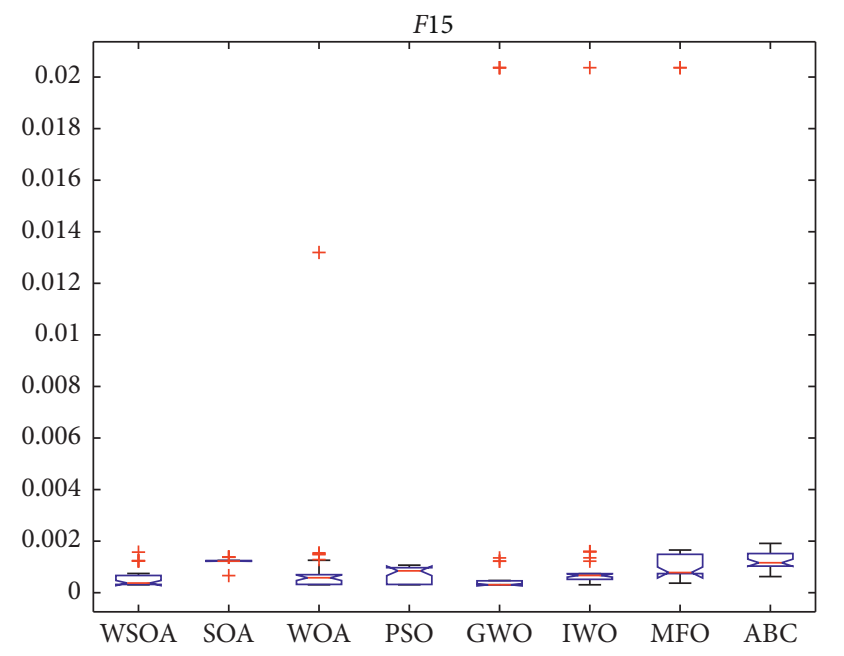

FIgURE 31: Variance analysis diagram of $f_{15}$.

ability of the algorithm. Generally speaking, the performance of the WSOA is far superior to the original SOA in testing 25 benchmark functions, which shows that the proposed algorithm has strong competitiveness.

5.5. Solving Engineering Optimization Problems. Engineering optimization problems are common in people's lives, but many optimization problems have complex constraints, especially the optimization of engineering structure design. In order to verify the performance of the WSOA in

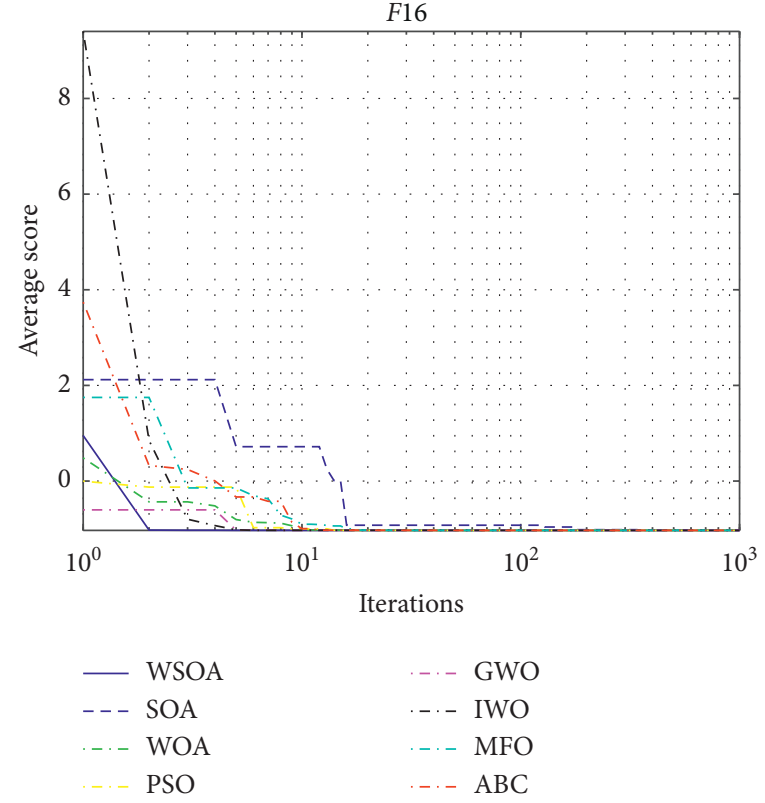

Figure 32: Convergence curve of $f_{16}$.

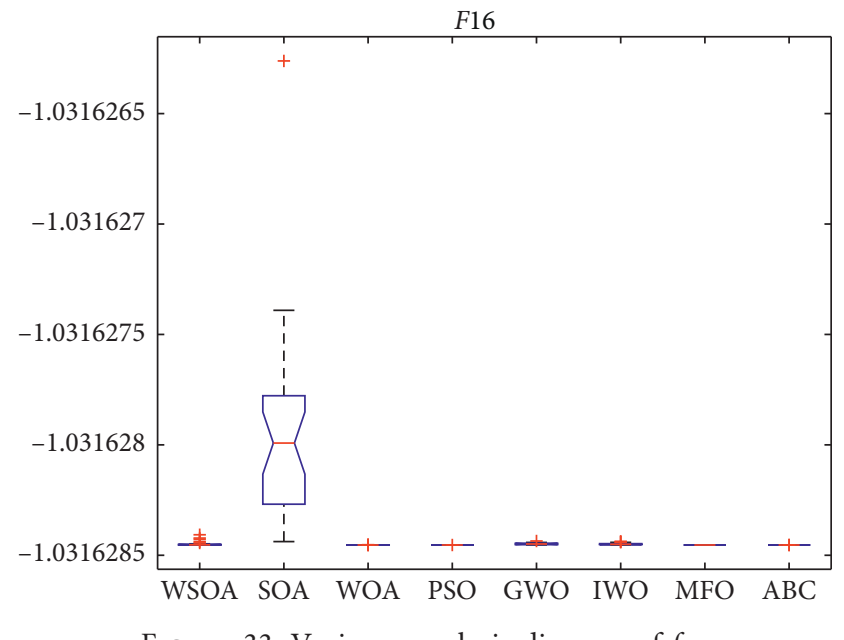

FIgURE 33: Variance analysis diagram of $f_{16}$.

solving the optimization problem of engineering structure design, the design problems such as tension/compression spring, welded beam, multiplate clutch separator, and pressure vessel are verified in this experiment.

5.5.1. Tension/Compression Spring Design Problem. In real life, the optimization goal of many problems is to minimize the weight of the problem. For example, the design problem of tension/compression spring is a common example. The variables of this problem include coil diameter $\left(x_{1}\right)$, spring 


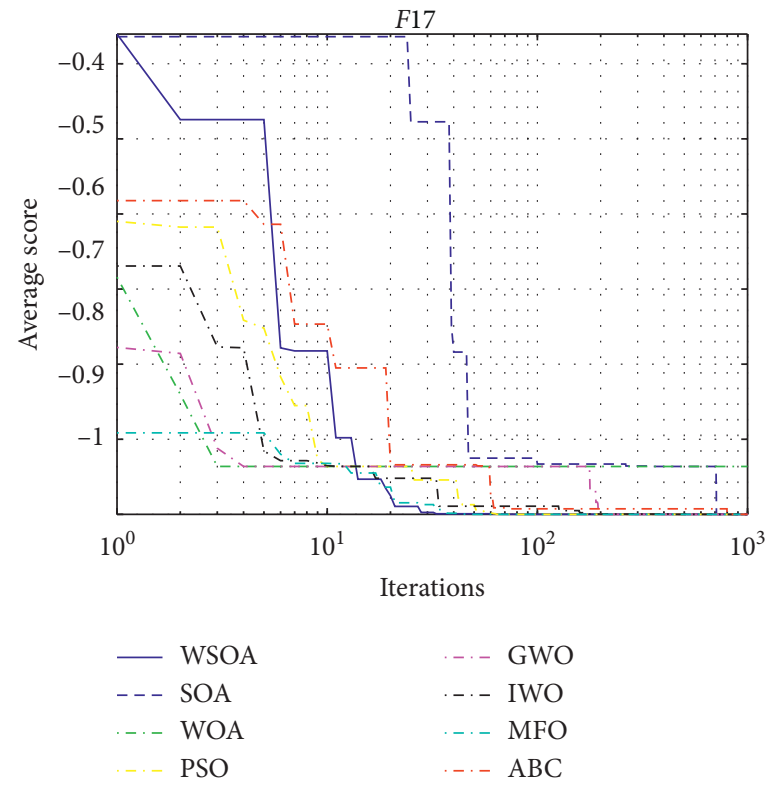

FIgURE 34: Convergence curve of $f_{17}$.

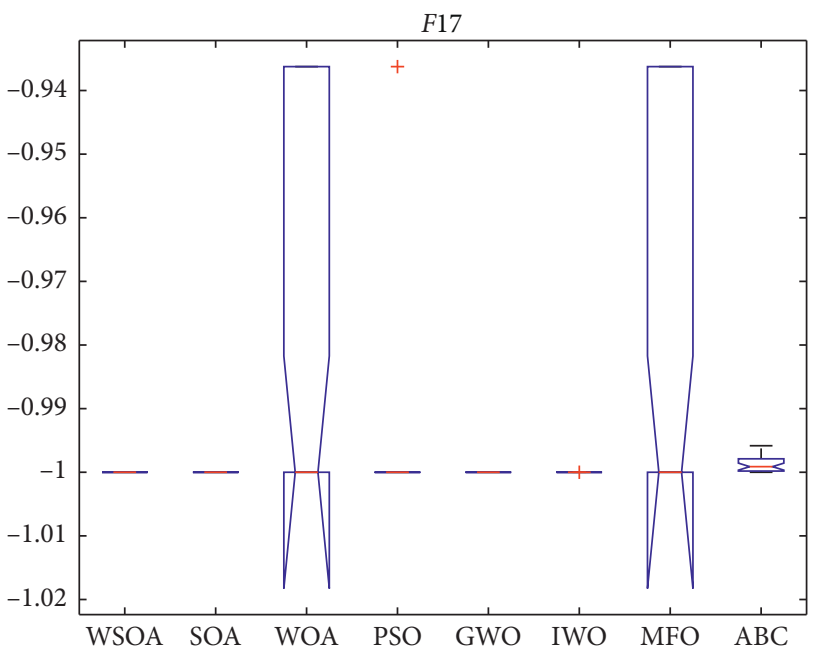

FIGURE 35: Variance analysis diagram of $f_{17}$.

coil diameter $\left(x_{2}\right)$, and the number of coils $\left(x_{3}\right)$. The constraint conditions are minimum deflection $\left(g_{1}(X)\right)$, shear stress $\left(g_{2}(X)\right)$, impact frequency $\left(g_{3}(X)\right)$, and outer diameter limit $\left(g_{4}(X)\right)$. The spring design pattern is shown in Figure 52, and the mathematical formula can be expressed as follows: consider: $\vec{x}=\left[x_{1} x_{2} x_{3}\right]=[\mathrm{d} D N]$,

minimize: $f(X)=\left(x_{3}+2\right) x_{2} x_{1}^{2}$,

$$
\begin{aligned}
g_{2}(X) & =\frac{4 x_{2}^{2}-x_{1} x_{2}}{12566\left(x_{2} x_{1}^{3}-x_{1}^{4}\right)}+\frac{1}{5180 x_{1}^{2}}-1 \leq 0 \\
g_{3}(X) & =1-\frac{140.45 x_{1}}{x_{2}^{2} x_{3}} \leq 0 \\
g_{4}(X) & =\frac{x_{1}+x_{2}}{1.5}-1 \leq 0 \\
0.05 & \leq x_{1} \leq 2 \\
0.25 & \leq x_{2} \leq 1.3 \\
2 & \leq x_{3} \leq 15
\end{aligned}
$$

Table 10 shows the comparison results of tension/ compression spring design problems. It can be seen from the table that the WSOA proposed in this paper has better optimization performance, and the optimal function value obtained in solving spring design problems is lower than 


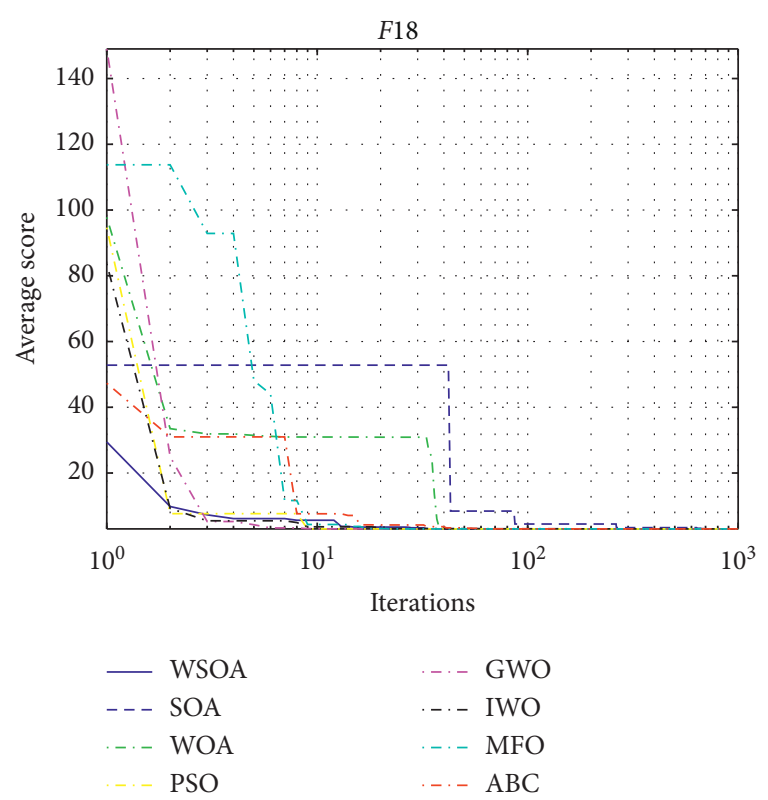

FIgURE 36: Convergence curve of $f_{18}$.

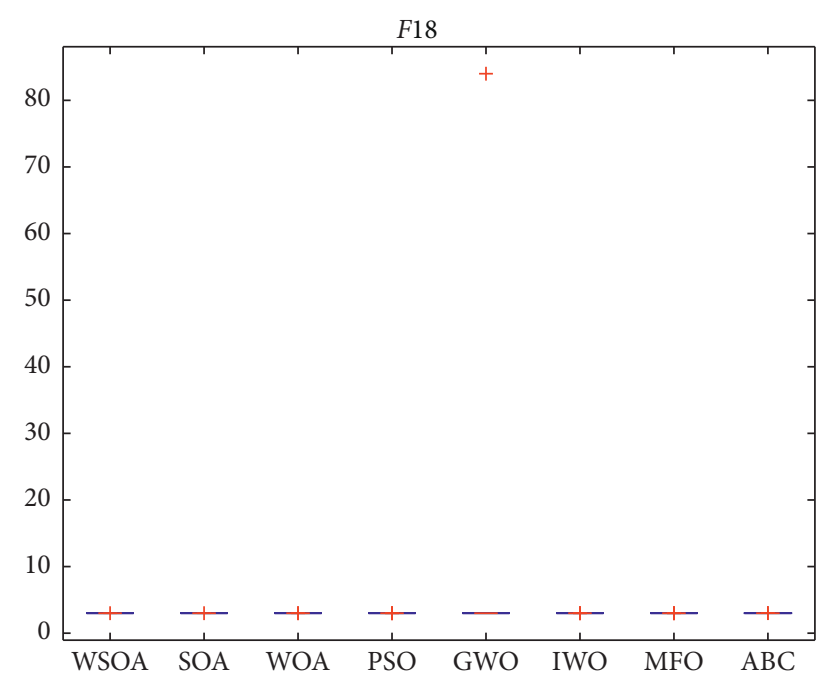

FIGURE 37: Variance analysis diagram of $f_{18}$.

those of previous studies. The algorithm of this experiment is the result of running independently for 30 times. The optimal fitness value of the WSOA is $f(x)=0.01267$, and the corresponding optimal solution is $\vec{x}=[0.0512,0.3441$, 12.0663]. It can be seen that the WSOA has better optimization accuracy in solving the design problem of tension/ compression spring.

5.5.2. Welded Beam Design Problem. The goal of welding beam design problem is to minimize the manufacturing cost, which must satisfy the related constraints such as shear stress $(\tau)$, beam bending stress $(\theta)$, bar buckling load $\left(P_{c}\right)$, beam end deflection $(\delta)$, normal stress $(\sigma)$, and boundary. The set variables include welded beam thickness $\left(x_{1}\right)$, welded joint length $\left(x_{2}\right)$, welded beam width $\left(x_{3}\right)$, and beam thickness

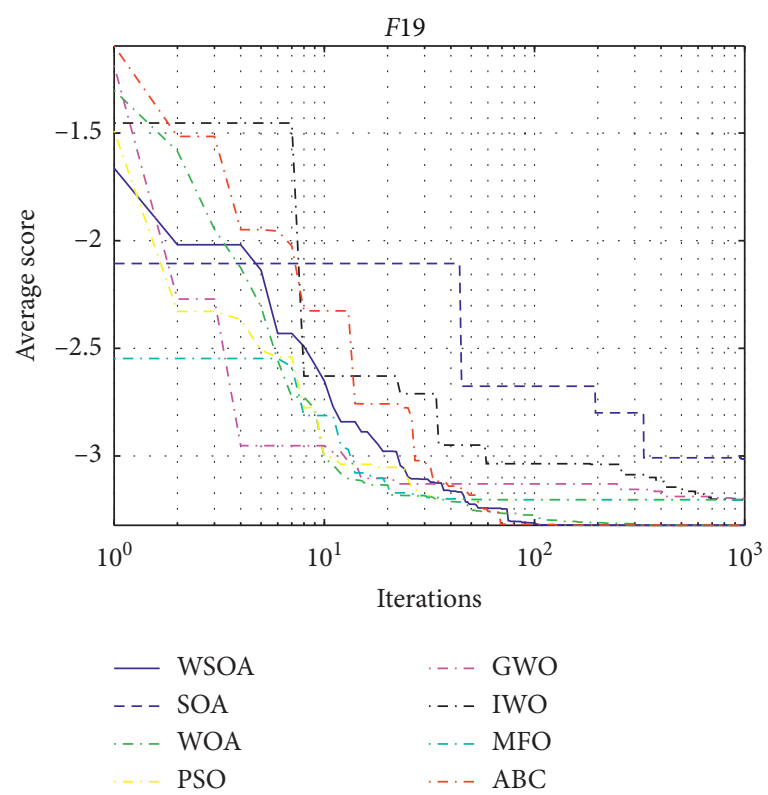

Figure 38: Convergence curve of $f_{19}$.

$\left(x_{4}\right)$. See Figure 53 for the design pattern of welded beam and the mathematical formula can be expressed as follows:

consider: $\vec{x}=\left[x_{1} x_{2} x_{3} x_{4}\right]=[$ hltb $]$,

minimize: $f(x)=1.10471 x_{1}^{2} x_{2}+0.04811 x_{3} x_{4}\left(14+x_{2}\right)$,

$$
\begin{aligned}
\text { s.t. } g_{1}(X) & =\tau(X)-\tau_{\max }, \\
g_{2}(X) & =\sigma(X)-\sigma_{\max }, \\
g_{4}(X) & =0.125-x_{1} \leq 0, \\
g_{5}(X) & =\delta(X)-0.25 \leq 0, \\
g_{6}(X) & =P-P_{c}(X) \leq 0, \\
g_{7}(X) & =0.10471 x_{1}^{2}+0.04811 x_{3} x_{4}\left(14+x_{2}\right)-5 \leq 0, \\
0.1 & \leq x_{1} \leq 2, \\
0.1 & \leq x_{2} \leq 10, \\
0.1 & \leq x_{3} \leq 10, \\
0.1 & \leq x_{4} \leq 2,
\end{aligned}
$$

where $\tau_{\max }$ is the maximum acceptable shear stress, $\sigma_{\max }$ is the maximum acceptable normal stress, and $P$ is the load. We have the following:

$$
\begin{aligned}
\tau(X) & =\sqrt{\tau_{1}^{2}+2 \tau_{1} \tau_{2}\left(\frac{x_{2}}{2 R}\right)+\tau_{2}^{2}}, \\
\tau_{1} & =\frac{P}{\sqrt{2} x_{1} x_{2}}, \\
\tau_{2} & =\frac{\mathrm{MR}}{J} \\
M & =P\left(L+\frac{x_{2}}{2}\right)
\end{aligned}
$$




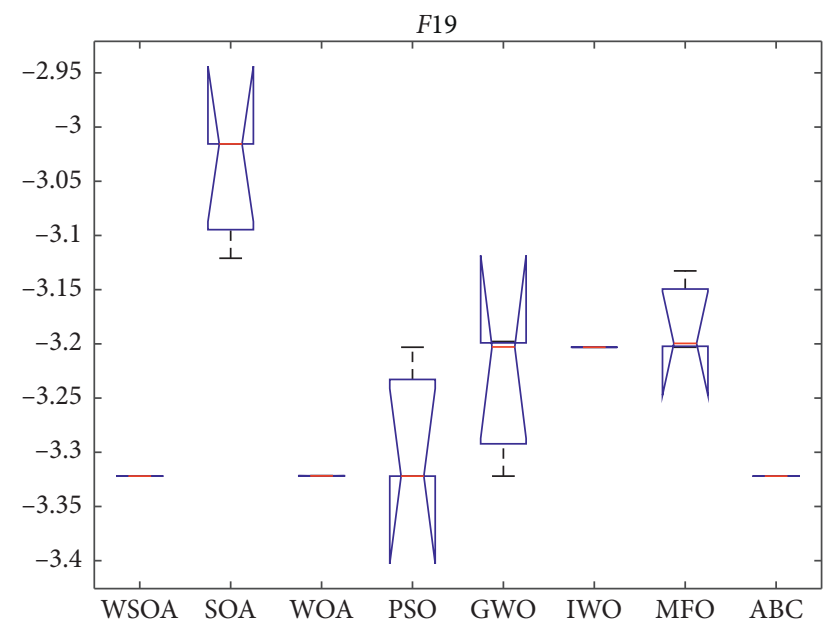

FigURe 39: Variance analysis diagram of $f_{19}$.
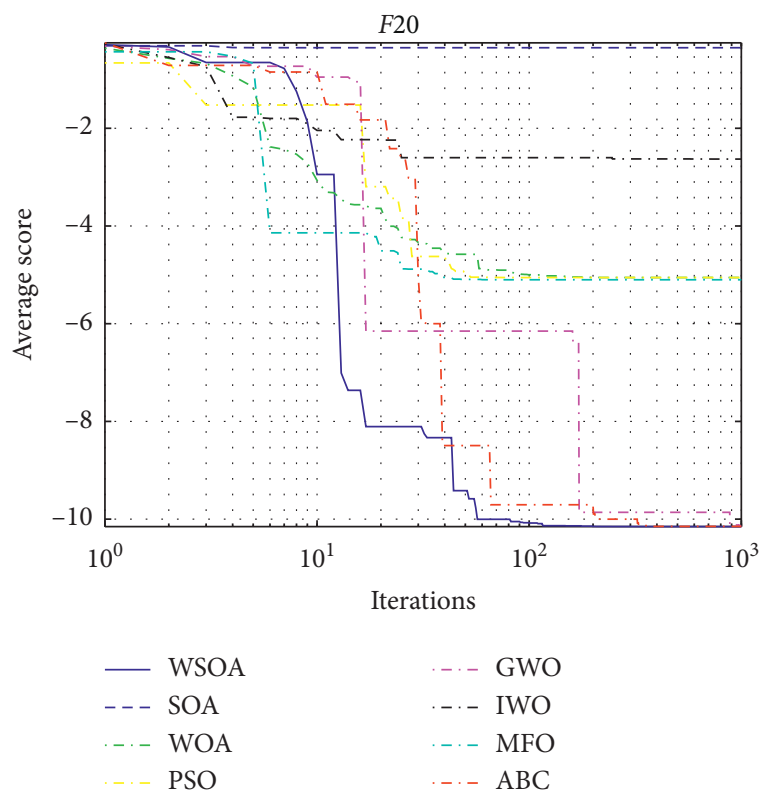

FIgURE 40: Convergence curve of $f_{20}$.

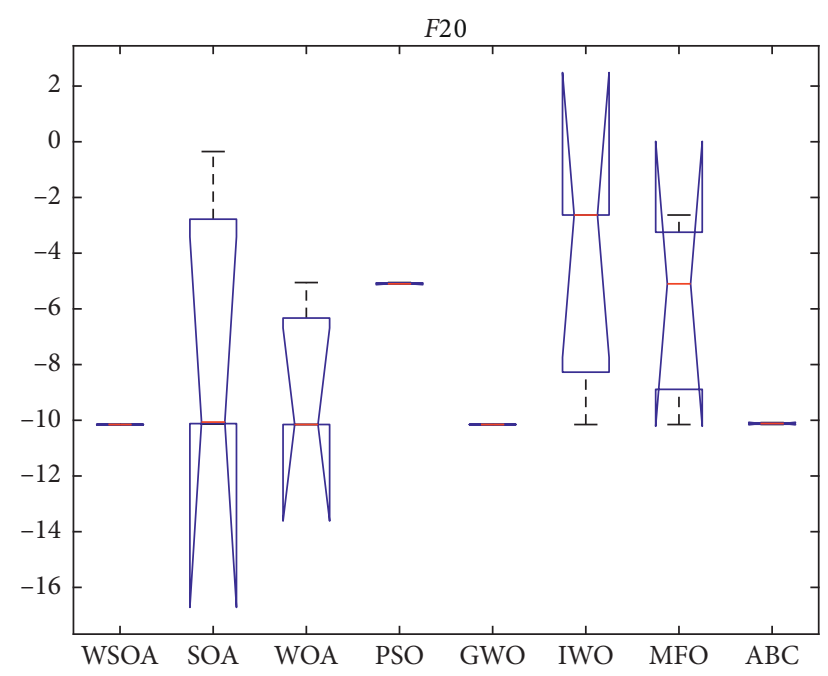

FIgURE 41: Variance analysis diagram of $f_{20}$.

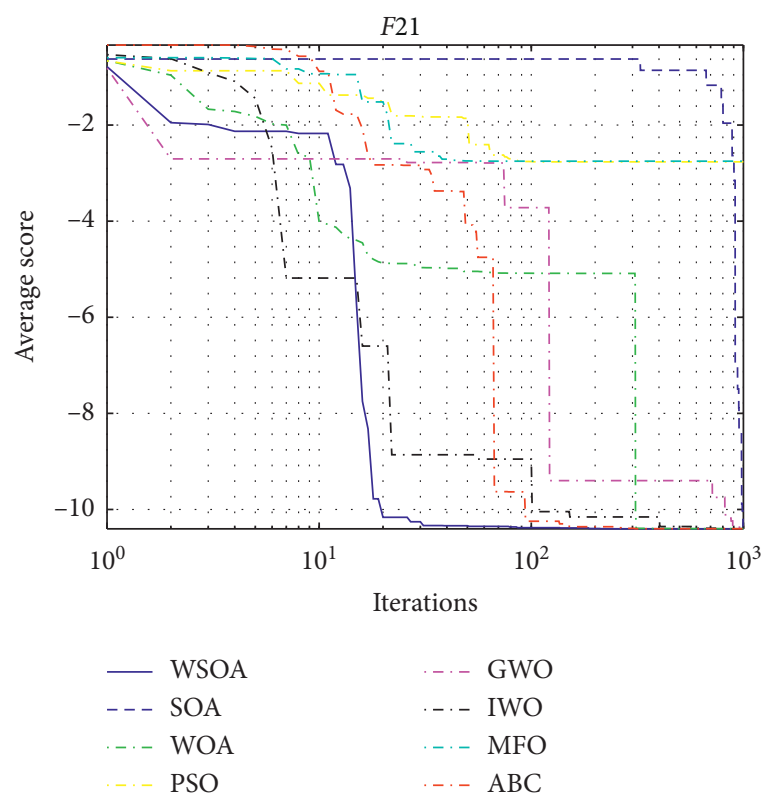

Figure 42: Convergence curve of $f_{21}$.

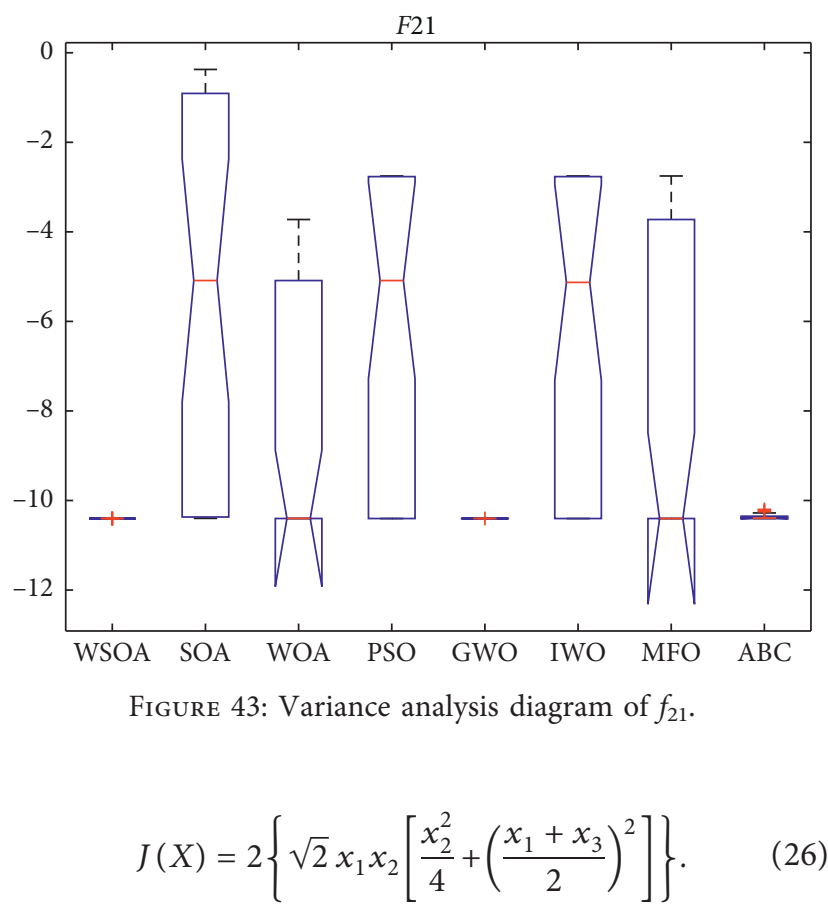

$M$ in equation (25) and $J(X)$ in equation (26) represent moment of inertia and polarity, respectively. The remaining parameters are shown in equation (27) to equation (31).

$$
\begin{aligned}
R & =\sqrt{\frac{x_{2}^{2}}{4}+\left(\frac{x_{1}+x_{3}}{2}\right)^{2}} . \\
\sigma(X) & =\frac{6 P L}{x_{4} x_{3}^{2}},
\end{aligned}
$$




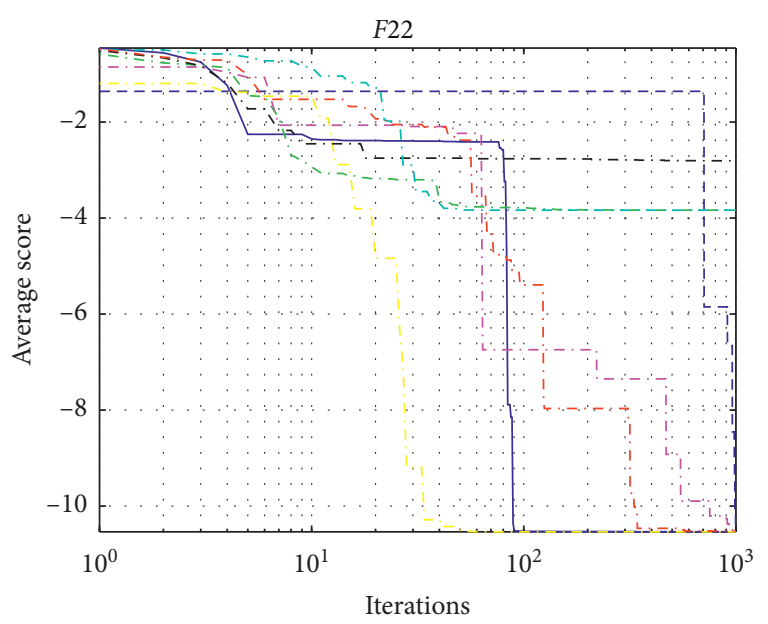

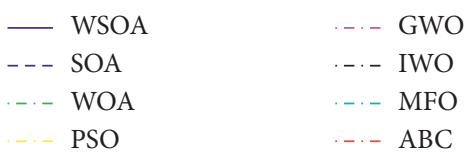

FIgURE 44: Convergence curve of $f_{22}$.

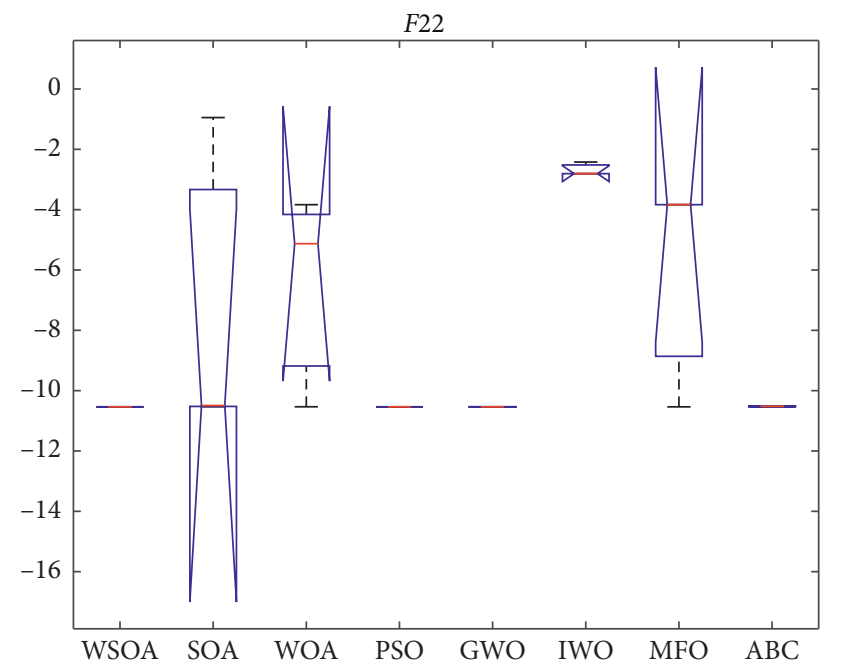

FIgURE 45: Variance analysis diagram of $f_{22}$.

$$
\begin{aligned}
\delta(X) & =\frac{6 P L^{3}}{E x_{3}^{3} x_{4}} \\
P_{c}(X) & =\frac{4.013 E \sqrt{x_{3}^{2} x_{4}^{6} / 36}}{L^{2}}\left(1-\frac{x_{3}}{2 L} \sqrt{\frac{E}{4 G}}\right), \\
G & =12 \times 10^{6} \mathrm{psi} \\
E & =30 \times 10^{6} \mathrm{psi}, \\
P & =6000 \mathrm{lb} \\
L & =14 \mathrm{in} .
\end{aligned}
$$

Table 11 shows the research results of welding beam design. It can be seen from the table that the function value of the WSOA in solving the design problem of welded beam

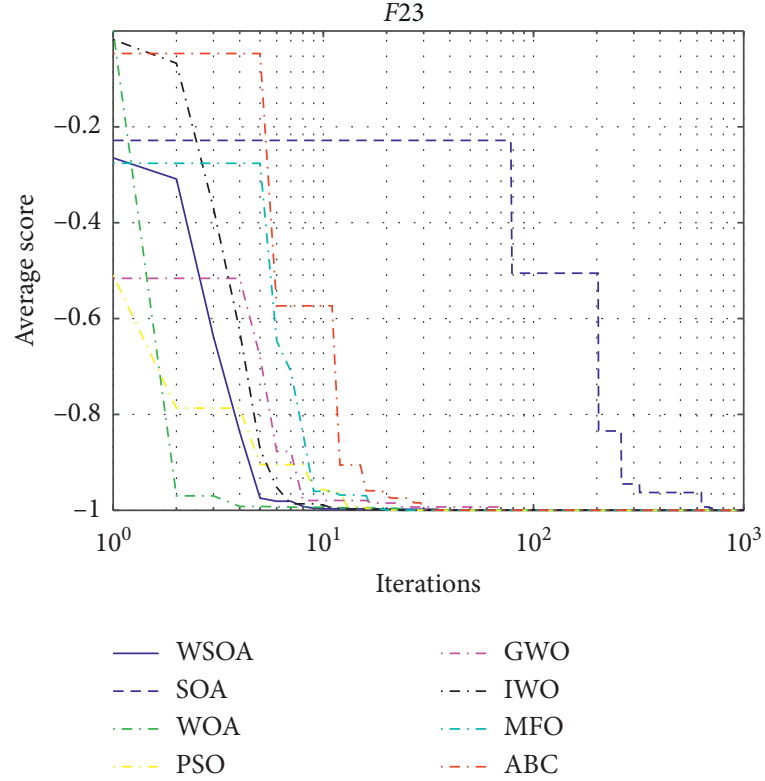

FIgURE 46: Convergence curve of $f_{23}$.

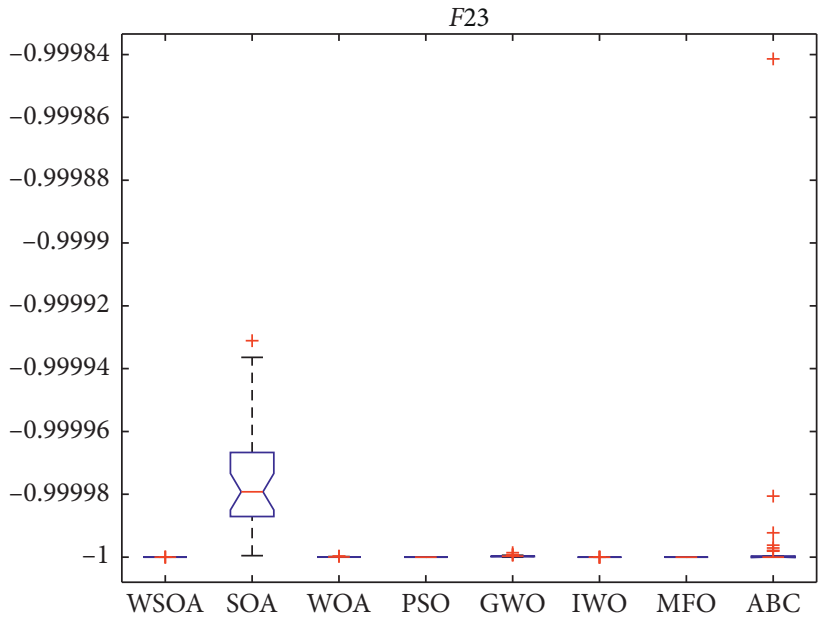

FIGURE 47: Variance analysis diagram of $f_{23}$.

is lower than that of previous research. The algorithm of this experiment is the result of running independently for 30 times. The optimal fitness value of the WSOA is $f(x)=1.7519$, and the corresponding optimal solution is $\vec{x}=[0.1919,3.7633,9.109,0.2054]$. Therefore, it can be shown that the WSOA has better optimization accuracy in solving the design problem of welded beams.

5.5.3. Multiplate Disc Clutch Brake Design Problem. The purpose of multiplate disc clutch brake is to optimize the total weight of multiplate disc clutch brake, in which the variables are driving force $(F)$, inner and outer radius ( $r i$ and $r 0)$, friction surface number $(Z)$, and disc thickness $(t)$. Because the problem contains eight different constraints, the feasible region in the solution space only accounts for $70 \%$, which makes it more difficult to solve the problem. See Figure 54 for the design pattern of multiplate disc clutch 


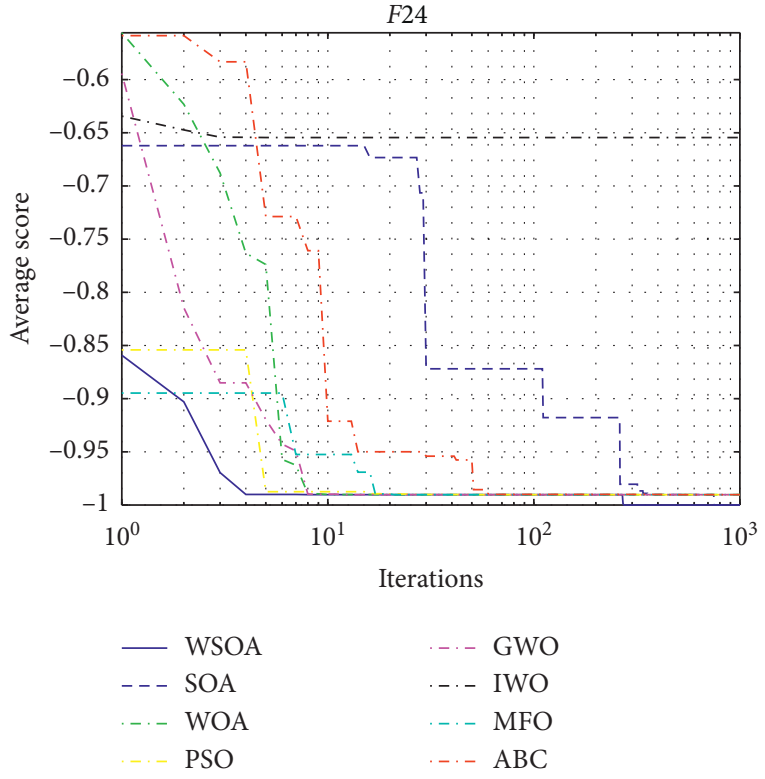

FIGURE 48: Convergence curve of $f_{24}$.

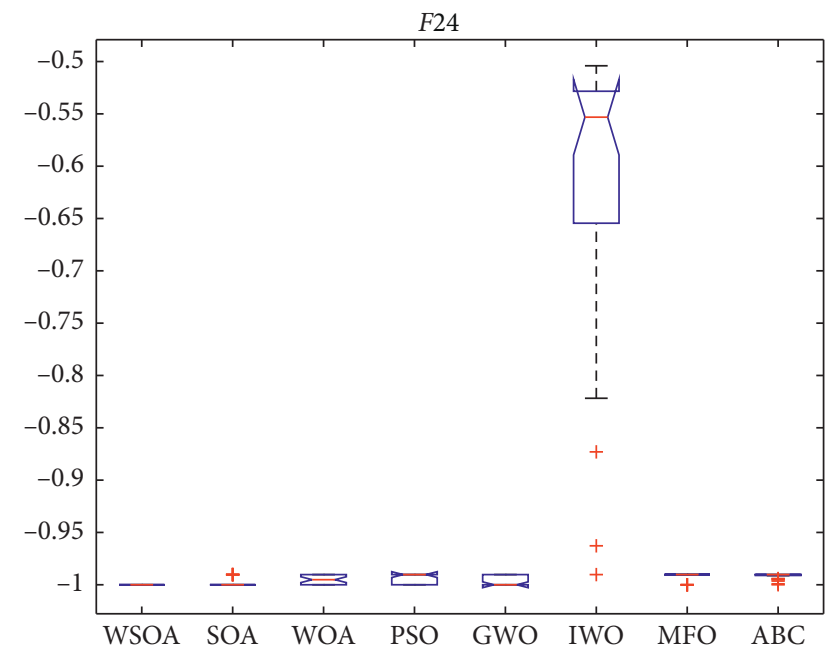

FIgURE 49: Variance analysis diagram of $f_{24}$.

brake, and the mathematical formula can be expressed as follows:

$$
\begin{aligned}
\text { minimize: } & f(x)=\pi\left(x_{2}^{2}-x_{1}^{2}\right) x_{3}\left(x_{5}+1\right) \rho, \\
\text { s.t. } g_{1}(x) & =x_{2}-x_{1}-\Delta R \geq 0, \\
g_{2}(x) & =L_{\max }-\left(x_{5}+1\right)\left(x_{3}+\delta\right) \geq 0, \\
g_{3}(x) & =P_{\max }-P_{r z} \geq 0, \\
g_{4}(x) & =P_{\max } v_{s r \max }-P_{r z} v_{s r} \geq 0, \\
g_{5}(x) & =v_{s r \max }-v_{s r} \geq 0, \\
g_{6}(x) & =T_{\max }-T \geq 0, \\
g_{7}(x) & =M_{h}-s M_{s} \geq 0, \\
g_{8}(x) & =T \geq 0,
\end{aligned}
$$

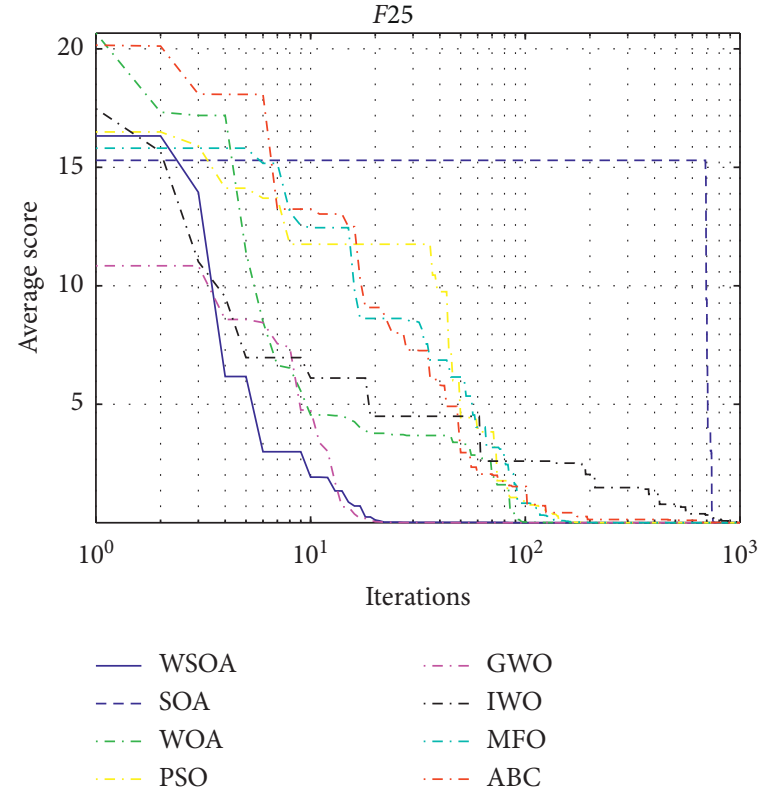

Figure 50: Convergence curve of $f_{25}$.

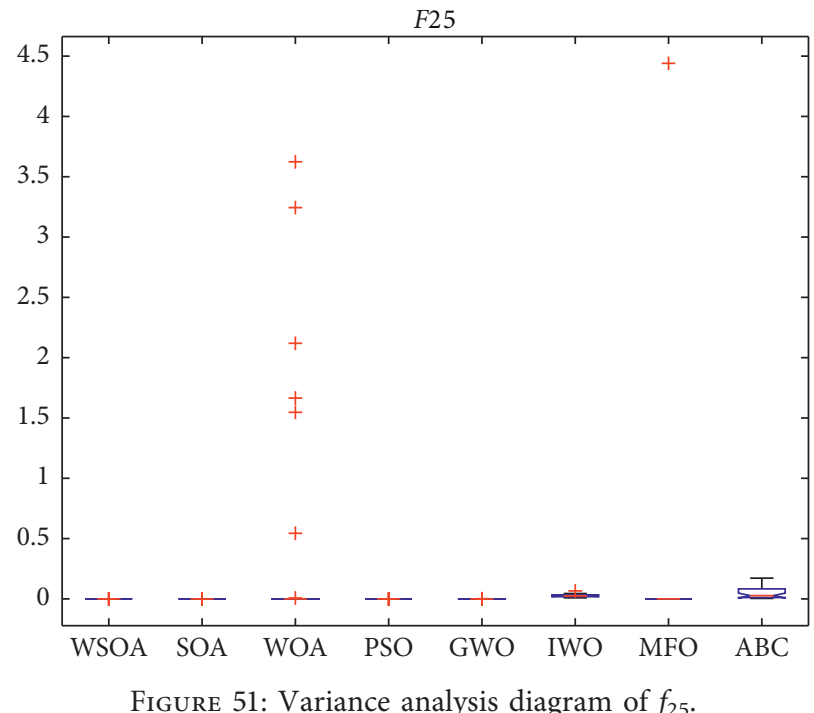

where $\quad M_{h}=(2 / 3) \mu x 4 \times 5\left(x_{2}^{3}-x_{1}^{3} / x_{2}^{2}-x_{1}^{2}\right) \quad$ and $w=(\pi n / 30) \mathrm{rad} / s$.

$$
\begin{aligned}
A & =\pi\left(x_{2}^{2}-x_{1}^{2}\right) \mathrm{mm}^{2}, \\
P_{r z} & =\frac{x_{4}}{A} \mathrm{~N} / \mathrm{mm}^{2}, \\
V_{s r} & =\frac{\pi R_{s r} n}{30} \mathrm{~mm} / \mathrm{s}, \\
R_{s r} & =\frac{2}{3} \frac{x_{2}^{2}-x_{1}^{2}}{x_{2}^{2} x_{1}^{2}} \mathrm{~mm}, \\
T & =\frac{I_{z} \pi n}{30\left(M_{h}+M_{f}\right)} \mathrm{mm},
\end{aligned}
$$


TABLE 8: Wilcoxon signed-rank test results between the WSOA and seven compared algorithms.

\begin{tabular}{|c|c|c|c|c|c|c|c|c|c|c|c|c|c|c|c|c|c|c|c|c|c|}
\hline \multirow{3}{*}{ No. } & \multicolumn{21}{|c|}{ WSOA versus } \\
\hline & \multicolumn{3}{|l|}{ SOA } & \multicolumn{3}{|c|}{ WOA } & \multicolumn{3}{|l|}{ PSO } & \multicolumn{3}{|c|}{ GWO } & \multicolumn{3}{|c|}{ IWO } & \multicolumn{3}{|c|}{ MFO } & \multicolumn{3}{|c|}{$\mathrm{ABC}$} \\
\hline & $P$ value & $\mathrm{H}$ & S & $P$ value & $\mathrm{H}$ & S & $P$ value & $\mathrm{H}$ & S & $P$ value & $\mathrm{H}$ & S & $P$ value & $\mathrm{H}$ & $\mathrm{S}$ & Pvalue & $\mathrm{H}$ & $\mathrm{S}$ & $P$ value & $\mathrm{H}$ & $S$ \\
\hline$f_{1}$ & $1.73 E-06$ & 1 & + & -11 & 1 & + & -11 & 1 & + & -11 & 1 & + & $2 E-11$ & 1 & + & $2 E-11$ & 1 & + & $2 E-11$ & 1 & + \\
\hline$f_{2}$ & $1.73 E-06$ & 1 & + & $3.47 E-10$ & 1 & + & $3.02 E-11$ & 1 & + & $3.02 E-11$ & 1 & + & $2 E-11$ & 1 & + & $3.02 E-11$ & 1 & + & $2 E-11$ & 1 & + \\
\hline$f_{3}$ & $1.73 E-06$ & 1 & + & $3.02 E-11$ & 1 & + & $3.02 E-11$ & 1 & + & $3.02 E-11$ & 1 & + & $3.02 E-11$ & 1 & + & $3.02 E-11$ & 1 & + & $.02 E-11$ & 1 & + \\
\hline$f_{4}$ & $1.73 E-06$ & 1 & + & $3.02 E-11$ & 1 & + & $3.02 E-11$ & 1 & + & $3.02 E-11$ & 1 & + & $3.02 E-11$ & 1 & + & $3.02 E-11$ & 1 & + & $3.02 E-11$ & 1 & + \\
\hline$f_{5}$ & $2.60 E-06$ & 1 & + & $6.05 E-07$ & 1 & + & $4.69 E-08$ & 1 & + & $1.03 E-06$ & 1 & + & $2.02 E-08$ & 1 & + & -11 & 1 & + & $E-11$ & 1 & + \\
\hline$f_{6}$ & $1.73 E-06$ & 1 & + & $3.02 E-11$ & 1 & + & $3.02 E-11$ & 1 & + & $5.57 E-10$ & 1 & + & $5.57 E-10$ & 1 & + & $3.99 E-04$ & 1 & + & $3.69 E-11$ & 1 & . \\
\hline$f_{7}$ & $1.36 E-05$ & 1 & + & $1.73 E-07$ & 1 & + & $3.02 E-11$ & 1 & + & $5.97 E-09$ & 1 & + & $3.02 E-11$ & 1 & + & $3.02 E-11$ & 1 & + & $3.02 E-11$ & 1 & + \\
\hline$f_{8}$ & $1.73 E-06$ & 1 & + & $3.37 E-04$ & 1 & + & $3.02 E-11$ & 1 & + & $3.02 E-11$ & 1 & + & $3.02 E-11$ & 1 & + & $3.02 E-11$ & 1 & + & $2.61 E-10$ & 1 & + \\
\hline$f_{9}$ & $1.25 E-01$ & 0 & $=$ & $1.00 E+00$ & 0 & $=$ & $1.21 E-12$ & 1 & + & $1.36 E-03$ & 1 & + & $1.21 E-12$ & 1 & + & $1.21 E-12$ & 1 & + & $1.21 E-12$ & 1 & + \\
\hline$f_{10}$ & $1.73 E-06$ & 1 & + & $1.01 E-08$ & 1 & + & $1.21 E-12$ & 1 & + & $2.05 E-13$ & 1 & + & $1.21 E-12$ & 1 & + & $1.21 E-12$ & 1 & + & $1.21 E-12$ & 1 & 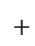 \\
\hline$f_{11}$ & $1.25 E-01$ & 0 & $=$ & $3.34 E-01$ & 0 & $=$ & $1.21 E-12$ & 1 & + & $2.79 E-03$ & 1 & + & $1.21 E-12$ & 1 & + & $1.21 E-12$ & 1 & + & $1.21 E-12$ & 1 & + \\
\hline$f_{12}$ & $1.73 E-06$ & 1 & + & $5.49 E-11$ & 1 & + & $1.86 E-01$ & 0 & $=$ & $3.02 E-11$ & 1 & + & $3.02 E-11$ & 1 & + & $5.09 E-08$ & 1 & + & $3.02 E-11$ & 1 & + \\
\hline$f_{13}$ & $1.73 E-06$ & 1 & + & $4.08 E-11$ & 1 & + & $9.59 E-01$ & 0 & $=$ & $5.57 E-10$ & 1 & + & $2.87 E-10$ & 1 & + & $1.00 E-03$ & 1 & + & $3.02 E-11$ & 1 & + \\
\hline$f_{14}$ & $8.13 E-01$ & 0 & $=$ & $1.70 E-02$ & 1 & + & $1.21 E-12$ & 1 & + & $1.44 E-03$ & 1 & + & $6.69 E-11$ & 1 & + & $3.08 E-01$ & 0 & $=$ & $3.34 E-03$ & 1 & + \\
\hline$f_{15}$ & $3.52 E-06$ & 1 & + & $2.52 E-01$ & 0 & $=$ & $2.34 E-01$ & 0 & $=$ & $3.56 E-04$ & 1 & + & $1.63 E-02$ & 1 & + & $4.12 E-06$ & 1 & + & $1.47 E-07$ & 1 & + \\
\hline$f_{16}$ & $1.92 E-06$ & 1 & + & $1.86 E-09$ & 1 & + & $1.72 E-12$ & 1 & + & $1.44 E-03$ & 1 & + & $4.86 E-03$ & 1 & + & $1.21 E-12$ & 1 & + & $1.75 E-11$ & 1 & + \\
\hline$f_{17}$ & $1.00 E+00$ & 0 & $=$ & $6.42 E-05$ & 1 & + & $3.34 E-01$ & 0 & $=$ & $1.00 E+00$ & 0 & $=$ & $1.21 E-12$ & 1 & + & $1.40 E-04$ & 1 & + & $1.21 E-12$ & 1 & + \\
\hline$f_{18}$ & $3.49 E-01$ & 0 & $=$ & $1.58 E-01$ & 0 & $=$ & $3.16 E-12$ & 1 & + & $2.17 E-01$ & 0 & $=$ & $3.20 E-09$ & 1 & + & $2.33 E-11$ & 1 & + & $1.78 E-04$ & 1 & + \\
\hline$f_{19}$ & $4.29 E-06$ & 1 & + & $1.76 E-01$ & 0 & $=$ & $1.07 E-04$ & 1 & + & $2.32 E-02$ & 1 & + & $3.79 E-01$ & 0 & $=$ & $1.54 E-01$ & 0 & $=$ & $1.61 E-10$ & 1 & + \\
\hline$f_{20}$ & $8.47 E-06$ & 1 & + & $3.50 E-09$ & 1 & + & $5.51 E-02$ & 0 & $=$ & $1.70 E-08$ & 1 & + & $1.60 E-03$ & 1 & + & $5.27 E-01$ & 0 & $=$ & $2.62 E-03$ & 1 & + \\
\hline$f_{21}$ & $1.73 E-06$ & 1 & + & $7.39 E-11$ & 1 & + & $7.86 E-03$ & 1 & + & $2.67 E-09$ & 1 & + & $2.51 E-02$ & 1 & + & $1.83 E-01$ & 0 & $=$ & $1.03 E-06$ & 1 & + \\
\hline$f_{22}$ & $1.36 E-05$ & 1 & + & $8.10 E-10$ & 1 & + & $2.22 E-02$ & 1 & + & $1.60 E-07$ & 1 & + & $1.54 E-01$ & 0 & $=$ & $6.16 E-03$ & 1 & + & $2.15 E-06$ & 1 & + \\
\hline$f_{23}$ & $1.73 E-06$ & 1 & + & $9.21 E-05$ & 1 & + & $1.21 E-12$ & 1 & + & $3.34 E-11$ & 1 & + & $9.71 E-01$ & 0 & $=$ & $1.21 E-12$ & 1 & + & $2.12 E-01$ & 0 & $=$ \\
\hline$f_{24}$ & $3.13 E-02$ & 1 & + & $1.23 E-05$ & 1 & + & $5.66 E-07$ & 1 & + & $3.13 E-04$ & 1 & + & $1.21 E-12$ & 1 & + & $8.99 E-11$ & 1 & + & $1.21 E-12$ & 1 & + \\
\hline$f_{25}$ & $1.73 E-06$ & 1 & + & $3.16 E-10$ & 1 & + & $3.02 E-11$ & 1 & + & $3.02 E-11$ & 1 & + & $3.02 E-11$ & 1 & + & $3.02 E-11$ & 1 & + & $3.02 E-11$ & 1 & + \\
\hline$w / t / 1$ & $20 / 5 /($ & & & $20 / 5 /($ & & & $20 / 5 /($ & & & $23 / 2 /($ & & & $22 / 3 /$ & & & $21 / 4 /($ & & & $24 / 1 /($ & & \\
\hline
\end{tabular}

TABLE 9: Friedman rank test results of the algorithm.

\begin{tabular}{lcc}
\hline Algorithms & Mean rank & Rank \\
\hline WSOA & 2.51 & 1 \\
SOA & 5.21 & 6 \\
WOA & 3.96 & 4 \\
PSO & 3.84 & 3 \\
GWO & 3.82 & 2 \\
IWO & 5.81 & 7 \\
MFO & 4.98 & 5 \\
ABC & 5.87 & 8 \\
\hline
\end{tabular}

$$
\begin{aligned}
\Delta r & =20 \mathrm{~mm}, \\
L_{\text {max }} & =30 \mathrm{~mm}, \\
\mu & =0.6, \\
T_{\text {max }} & =15 \mathrm{~s}, \\
\mu & =0.5, \\
s & =1.5, \\
M_{s} & =40 \mathrm{Nm}, \\
p_{\text {max }} & =1 \mathrm{Mp}, \\
\rho & =0.0000078 \mathrm{~kg} / \mathrm{mm}^{3},
\end{aligned}
$$

$$
\begin{aligned}
v_{s r \text { max }} & =10 \mathrm{~m} / \mathrm{s}, \\
\delta & =0.5 \mathrm{~mm}, \\
s & =1.5, \\
T_{\text {max }} & =15 \mathrm{~s}, \\
n & =250 \mathrm{rpm}, \\
I_{z} & =55 \mathrm{~kg} / \mathrm{m}^{2}, \\
M_{s} & =40 \mathrm{Nm}, \\
M_{f} & =3 \mathrm{Nm},
\end{aligned}
$$$$
60 \leq x_{1} \leq 80,
$$$$
90 \leq x_{2} \leq 110 \text {, }
$$$$
1 \leq x_{3} \leq 3
$$$$
60 \leq x_{4} \leq 1000
$$$$
2 \leq x_{5} \leq 9,
$$

$i=1,2,3,4,5$. 


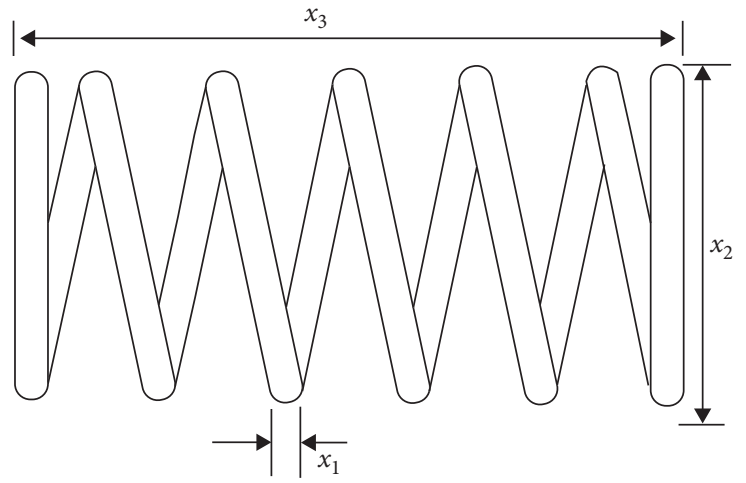

FIGURE 52: Structure of the TCS design.

TABLE 10: Comparative results for the tension/compression spring problem.

\begin{tabular}{lcccc}
\hline \multirow{2}{*}{ Algorithms } & \multicolumn{5}{c}{ Optimum variables } & Optimum weight \\
& $\mathrm{D}$ & $\mathrm{D}$ & $\mathrm{N}$ & \\
\hline WSOA & 0.0512 & 0.3441 & 12.0663 & $\mathbf{0 . 0 1 2 6 7}$ \\
GDA [38] & 0.0516925 & 0.3568108 & 11.2835059 & 0.012875 \\
GA [1] & 0.05148 & 0.351661 & 11.632201 & 0.0127048 \\
GPSO [39] & 0.0517 & 0.3573 & 11.254 & 0.0127 \\
SES [40] & $\mathrm{N} / \mathrm{A}$ & $\mathrm{N} / \mathrm{A}$ & $\mathrm{N} / \mathrm{A}$ & 0.012732 \\
SCPSO & 0.051688 & 0.356705 & 11.289687 & 0.0127576 \\
[41] & & & & \\
AATM & 0.051813 & 0.35969 & 11.119253 & 0.0127081 \\
[42] & & & & \\
UABC [43] & 0.051691 & 0.356769 & 11.285988 & 0.012683 \\
MVO [44] & 0.05 & 0.315956 & 14.22623 & 0.0144644 \\
TEO [45] & 0.051775 & 0.358792 & 11.16839 & 0.012685 \\
MGWO & 0.051334 & 0.348197 & 11.80809 & 0.0126799 \\
[46] & & & & \\
EEGWO & 0.051673 & 0.35634 & 11.3113 & 0.012685 \\
[47] & & & &
\end{tabular}

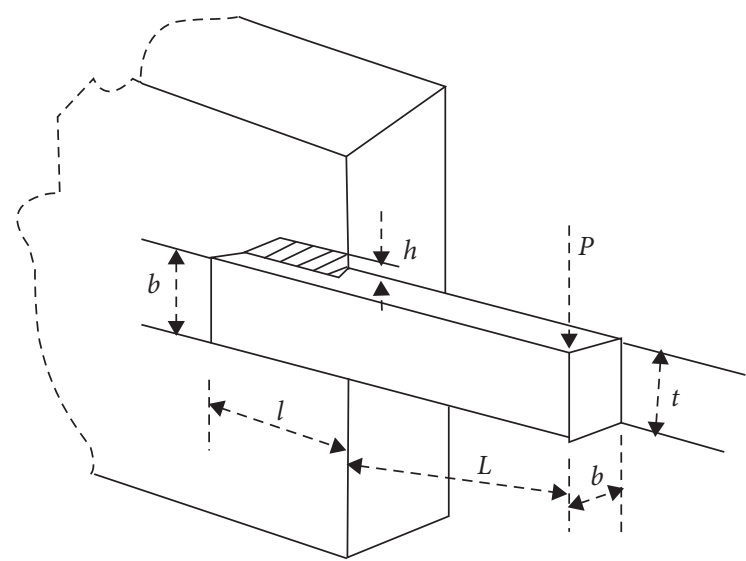

FIgURE 53: The structure of welded beam design.

The comparison results of design problems of multiplate disc clutch brake are shown in Table 12. It can be seen from the table that the WSOA shows superiority in solving this problem, and the obtained function value is lower than those of previous studies. The experiment was run independently for 30 times. The optimal fitness value of the algorithm is
TABle 11: Comparative results for the welded beam design problem.

\begin{tabular}{|c|c|c|c|c|c|}
\hline \multirow{2}{*}{ Algorithms } & \multicolumn{4}{|c|}{ Optimum variables } & \multirow{2}{*}{$\begin{array}{l}\text { Optimum } \\
\text { cost }\end{array}$} \\
\hline & $h$ & $L$ & $T$ & $b$ & \\
\hline$v$ & 0.1919 & 3.76 & 9.10 & 205 & 1.7519 \\
\hline$[48]$ & 0.2 & & 8.2 & 0.2 & 2.442 \\
\hline 49] & 0.247 & & 8.2 & 0.249 & 2.4148 \\
\hline SCA [50] & 0.244 & 6.2 & 8.2 & 0.2446 & 2.3854 \\
\hline BFOA & 0.205 & 3.47 & 9.0 & 0.2 & 68 \\
\hline & 0.2 & & 8.2 & 0.2 & \\
\hline $\mathrm{T}-$ & 0.24 & 6.12 & 8.25 & 0.2 & 2.3811 \\
\hline FSA [54] & 0.2444 & 6.1258 & 8.2939 & 0.2444 & 2.3811 \\
\hline GSA [55] & 0.182129 & 3.856979 & 10 & 0.202376 & 1.879952 \\
\hline & 0.2442 & 6.2231 & 8.2915 & 0.2443 & 2.3807 \\
\hline SBO [57] & 0.21421 & 3.4924 & 8.55771 & 0.22945 & 1.8496 \\
\hline
\end{tabular}

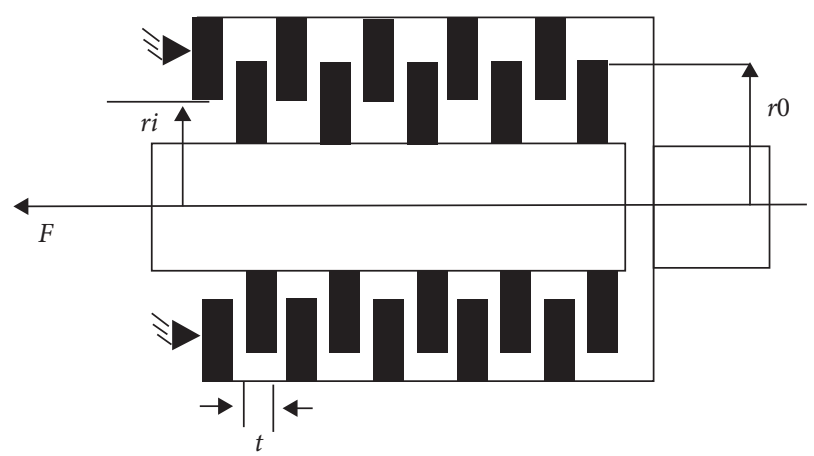

FIGURE 54: Multiplate disc clutch brake problem.

$f(x)=0.23525$, and the optimal solution is $\vec{x}=[69.9996$, $90,1,600,2]$. It can be shown that the WSOA has good optimization accuracy in solving the design problem of multiplate disc clutch brake.

5.5.4. Pressure Vessel Design Problem. The optimization purpose of pressure vessel problem is to minimize the total cost. Constraints include material cost, forming cost, and welding cost. The variables of this problem are shell thickness $\left(T_{s}\right)$, head thickness $\left(T_{h}\right)$, inner diameter $(R)$, and cylindrical section length $(L)$ [61]. See Figure 55 for the 
TABLe 12: Comparative results for multiplate disc clutch brake problem.

\begin{tabular}{lcccccc}
\hline Algorithms & $r i\left(x_{1}\right)$ & $r 0\left(x_{2}\right)$ & $t\left(x_{3}\right)$ & $F\left(x_{4}\right)$ & $Z\left(x_{5}\right)$ & Optimal cost \\
\hline WSOA & 69.9996 & 90 & 1 & 600 & 2 & $\mathbf{0 . 2 3 5 2 5}$ \\
TLBO [58] & 70 & 90 & 1 & 810 & 3 & 0.313656 \\
WCA [59] & 70 & 90 & 1 & 910 & 3 & 0.313656 \\
PVS [60] & 70 & 90 & 1 & 980 & 3 & 0.31366 \\
HHO [58] & 69.9999999992493 & 90 & 1 & 1000 & 3 & 0.259768993 \\
\hline
\end{tabular}

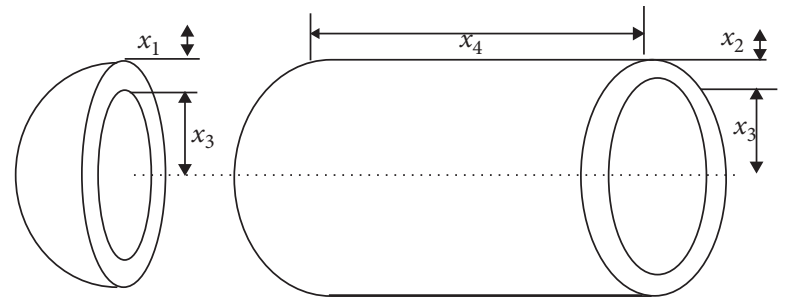

Figure 55: Pressure vessel design and its features.

TABLE 13: Comparative results for the pressure vessel design problem.

\begin{tabular}{|c|c|c|c|c|c|}
\hline \multirow{2}{*}{ Algorithms } & \multicolumn{4}{|c|}{ Optimum variables } & \multirow{2}{*}{ Optimum cost } \\
\hline & $T_{s}$ & $T_{h}$ & $R$ & $L$ & \\
\hline WSOA & 0.8056 & 0.4081 & 41.7401 & 181.1285 & 5964.6114 \\
\hline GWO [4] & 0.8125 & 0.4345 & 42.089181 & 176.758731 & 6051.5639 \\
\hline MFO [5] & 0.8125 & 0.4375 & 42.098445 & 176.636596 & 6059.7143 \\
\hline CPSO [62] & 0.8125 & 0.4375 & 42.091266 & 176.7465 & 6061.0777 \\
\hline HAIS-GA [63] & 0.8125 & 0.4375 & 42.0931 & 176.7031 & 6060.367 \\
\hline ES [64] & 0.8125 & 0.4375 & 42.098087 & 176.640518 & 6059.745 \\
\hline $\mathrm{CDE}[65]$ & 0.8125 & 0.4375 & 42.098411 & 176.63769 & 6059.734 \\
\hline BA [66] & 0.8125 & 0.4375 & 42.098445 & 176.636595 & 6059.7143 \\
\hline WOA [6] & 0.8125 & 0.4375 & 42.0982699 & 176.638998 & 6059.741 \\
\hline DTS-GA [67] & 0.8125 & 0.4375 & 42.097398 & 176.654047 & 6059.9463 \\
\hline GeneAS [68] & 0.9375 & 0.5 & 48.329 & 112.679 & 6410.3811 \\
\hline WEO [69] & 0.8125 & 0.4375 & 42.098444 & 176.636622 & 6059.71 \\
\hline HHO [70] & 0.81758383 & 0.4072927 & 42.09174576 & 176.7196352 & 6000.46259 \\
\hline
\end{tabular}

design of pressure vessel, and the mathematical formula can be expressed as follows:

consider: $Z=\left[z_{1}, z_{2}, z_{3}, z_{4}\right]=[h, l, t, b]$,

minimize: $f(x)=0.6224 x_{1} x_{3} x_{4}+1.7781 x_{2} x_{2}^{3}$

$$
+3.1661 x_{1}^{2} x_{4}+19.84 x_{1}^{2} x_{3}
$$

subject to

$$
\begin{aligned}
& g_{1}(x)=-x_{1}+0.0193 x_{3} \leq 0, \\
& g_{2}(x)=-x_{3}+0.00954 z_{3} \leq 0 \\
& g_{3}(x)=-\pi x_{3}^{2} x_{4}-\frac{4}{3} \pi x_{3}^{3}+1,296,00 \leq 0, \\
& g_{4}(x)=x_{4}-240 \leq 0
\end{aligned}
$$

where $0 \leq x_{1}, x_{2} \leq 99,0 \leq x_{3}$, and $x_{4} \leq 200$.
Table 13 shows the research results of the WSOA and other algorithms on pressure vessel design. It can be seen from the table that the results obtained by the WSOA algorithm are better than those obtained by other algorithms, in which the optimal fitness value is $f(x)=5964.6114$ and the optimal solution is $\vec{x}=[0.8056,0.4081,41.7401$, 181.1285]. It is shown that the algorithm has better optimization performance and accuracy in solving the design problem of pressure vessels.

\section{Research Limitations}

Although the above experimental results show that the WSOA has better performance than most comparative algorithms, it also has some shortcomings. There is a room for improvement in the performance of the WSOA. For example, in the unimodal test function, it is found that the number of theoretical optimum values obtained by the WSOA is small, which shows that the exploitation ability of the WSOA can be further improved in the test unimodal function. After testing the multimodal test function, it is 
found that the number of theoretical optimal values obtained is small, which indicates that the exploration and exploitation ability of the WSOA under multiple optimal solutions needs to be further improved. Moreover, the WSOA has not been further verified in a higher dimension. Finally, because the WSOA combines the WOA with the SOA, the structure of this algorithm is more complex than the original algorithm, so the flexibility of the WSOA is worse than that of the original algorithm.

\section{Conclusion and Future Work}

In this paper, an improved seagull optimization algorithm (WSOA) is proposed to solve the global optimization problem. The combination of contraction and encirclement mechanism of the WOA and spiral attack behavior of the SOA can improve the calculation accuracy of the SOA and balance the exploitation and exploration capabilities more effectively. In addition, introducing levy flight strategy into the search formula can avoid premature convergence of the SOA. In the aspect of performance evaluation, 25 benchmark test functions are used for verification. Comparing the performance of the WSOA with the performances of seven famous metaheuristic algorithms, the results show that the algorithm has strong competitiveness. Statistical test analysis and results comparison show that the improved SOA has better performance than the SOA. In this paper, four engineering examples are also used to test the performance of the proposed algorithm, which proves that the WSOA has better performance.

For the WSOA, further research is needed in future work. First of all, the WSOA can be applied to target allocation problem and more constrained engineering examples. Second, the WSOA is applied to solve real-life problems, such as medical data.

\section{Data Availability}

The data used to support the findings of this study are available from the corresponding author upon request.

\section{Conflicts of Interest}

The authors declare no conflicts of interest.

\section{Acknowledgments}

This work was supported by the National Natural Science Foundation of China (no. 11961006) and Guangxi Natural Science Foundation (no. 2020GXNSFAA159100).

\section{References}

[1] M. Srinivas and L. M. Patnaik, "Adaptive probabilities of crossover and mutation in genetic algorithms," IEEE Transactions on Systems Man \& Cybernetics, vol. 24, no. 4, pp. 656-667, 2002.

[2] J. Kennedy and R. Eberhart, Particle Swarm Optimization. Icnn95-International Conference on Neural Networks, IEEE, London, UK, 2002.
[3] J. Soares, T. Sousa, Z. A. Vale, H. Morais, and P. Faria, "Ant colony search algorithm for the optimal power flow problem," 2011.

[4] S. Mirjalili, S. M. Mirjalili, and A. Lewis, "Grey wolf optimizer," Advances in Engineering Software, vol. 69, no. 3, pp. 46-61, 2014.

[5] S. Mirjalili, "Moth-flame optimization algorithm: a novel nature-inspired heuristic paradigm," Knowledge-Based Systems, vol. 89, no. 2, pp. 228-249, 2015.

[6] S. Mirjalili and A. Lewis, "The whale optimization algorithm," Advances in Engineering Software, vol. 95, no. 95, pp. 51-67, 2016.

[7] M. Jain, V. Singh, and A. Rani, "A novel nature-inspired algorithm for optimization: squirrel search algorithm," Swarm and Evolutionary Computation, vol. 44, 2018.

[8] R. Masadeh, B. A. Mahafzah, and A. A. Sharieh, "Sea lion optimization algorithm," International Journal of Advanced Computer Science and Applications, vol. 10, no. 5, pp. 388-395, 2019.

[9] H. A. Alsattar, A. A. Zaidan, and B. B. Zaidan, "Novel metaheuristic bald eagle search optimisation algorithm," Artificial Intelligence Review, vol. 53, no. 6, 2020.

[10] M. Khishe and M. R. Mosavi, "Chimp optimization algorithm," Expert Systems with Applications, vol. 149, 2020.

[11] Anita and A. Yadav, "Aefa: artificial electric field algorithm for global optimization," Swarm and Evolutionary Computation, vol. 14, 2019.

[12] A. R. Mehrabian and C. Lucas, "A novel numerical optimization algorithm inspired from weed colonization," Ecological Informatics, vol. 1, no. 4, pp. 355-366, 2006.

[13] D. Karaboga and B. Basturk, "A powerful and efficient algorithm for numerical function optimization: artificial bee colony (abc) algorithm," Journal of Global Optimization, vol. 39, no. 3, pp. 459-471, 2007.

[14] D. H. Wolpert and W. G. Macready, "No free lunch theorems for optimization," IEEE Transactions on Evolutionary Computation, vol. 1, no. 1, pp. 67-82, 1997.

[15] T. O. Ting, X. S. Yang, S. Cheng, and K. Huang, "Hybrid metaheuristic algorithms: past, present, and future," 2015.

[16] S. Gupta and K. Deep, "A novel random walk grey wolf optimizer," Swarm and Evolutionary Computation, vol. 34, pp. 101-112, 2018.

[17] S. Gupta, K. Deep, A. A. Heidari, H. Moayedi, and H. Chen, "Harmonized salp chain-built optimization," Engineering with Computers, vol. 34, pp. 1-31, 2019.

[18] S. Gupta and K. Deep, "A memory-based grey wolf optimizer for global optimization tasks," Applied Soft Computing, vol. 93, 2020.

[19] S. Gupta, K. Deep, and A. P. Engelbrecht, "A memory guided sine cosine algorithm for global optimization," Engineering Applications of Artificial Intelligence, vol. 93, p. 103718, 2020.

[20] F. A. Hashim, K. Hussain, E. H. Houssein, M. S. Mabrouk, and W. Al-Atabany, "Archimedes optimization algorithm: a new metaheuristic algorithm for solving optimization problems," Applied Intelligence, vol. 34, pp. 1-21, 2020.

[21] A. G. Hussien, A. E. Hassanien, E. H. Houssein, M. Amin, and A. T. Azar, "New binary whale optimization algorithm for discrete optimization problems," Engineering Optimization, vol. 52, no. 6, pp. 945-959, 2020.

[22] S. Gupta and K. Deep, "A hybrid self-adaptive sine cosine algorithm with opposition based learning," Expert Systems With Applications, vol. 119, pp. 210-230, 2019. 
[23] S. Gupta and K. Deep, "Hybrid grey wolf optimizer with mutation operator," Advances in Intelligent Systems and Computing, vol. 34, no. 2, pp. 961-968, 2019.

[24] G. Dhiman and V. Kumar, "Seagull optimization algorithm: theory and its applications for large-scale industrial engineering problems," Knowledge-Based Systems, vol. 165, pp. 169-196, 2019.

[25] H. Jiang, Y. Yang, W. Ping, and Y. Dong, “A novel hybrid classification method based on the opposition-based seagull optimization algorithm," IEEE Access, vol. 8, pp. 100778-100790, 2020.

[26] H. Jia, Z. Xing, and W. Song, "A new hybrid seagull optimization algorithm for feature selection," IEEE Access, vol. 7, pp. 49614-49631, 2019.

[27] G. Dhiman, K. K. Singh, A. Slowik et al., "EMoSOA: a new evolutionary multi-objective seagull optimization algorithm for global optimization," International Journal of Machine Learning and Cybernetics, vol. 34, pp. 1-26, 2020.

[28] N. Panagant, N. Pholdee, S. Bureerat, A. R. Yild1z, and S. M. Sait, "Seagull optimization algorithm for solving real-world design optimization problems," Materials Testing, vol. 62, no. 6, pp. 640-644, 2020.

[29] X.-S. Yang, "Nature-inspired metaheuristic algorithms," 2010.

[30] X. Yao, Y. Liu, and G. Lin, "Evolutionary programming made faster," IEEE Transactions on Evolutionary Computation, vol. 3, no. 2, pp. 82-102, 1999.

[31] J. G. Digalakis and K. G. Margaritis, "On benchmarking functions for genetic algorithms," International Journal of Computer Mathematics, vol. 77, no. 4, pp. 481-506, 2001.

[32] X. S. Yang and X. She, "Firefly algorithm, stochastic test functions and design optimisation," International Journal of Bio-Inspired Computation, vol. 2, no. 2, pp. 78-84, 2010.

[33] J. Derrac, S. García, and F. Herrera, "A practical tutorial on the use of nonparametric statistical tests as a methodology for comparing evolutionary and swarm intelligence algorithms," Swarm and Evolutionary Computation, vol. 1, no. 1, pp. 3-18, 2011.

[34] M. Molina, I. Aljarah, A. A. Heidari et al., "Binary dragonfly optimization for feature selection using time-varying transfer functions," Knowledge-Based Systems, vol. 161, no. 1, pp. 185-204, 2018.

[35] P. Martinez-Penaloza and G. Maria-Guadalupe, "Immune generalized differential evolution for dynamic multi-objective environments: an empirical study," Knowledge Based Systems, vol. 6, 2018.

[36] J. Yi, L. Gao, X. Li, C. A. Shoemaker, and C. Lu, “An on-line variable-fidelity surrogate-assisted harmony search algorithm with multi-level screening strategy for expensive engineering design optimization," Knowledge-Based Systems, vol. 170, no. 15 , pp. 1-19, 2019.

[37] D. W. Zimmerman and B. D. Zumbo, "Relative power of the wilcoxon test, the friedman test, and repeated-measures anova on ranks," The Journal of Experimental Education, vol. 62, no. 1, pp. 75-86, 1993.

[38] A. Baykasoglu, "Design optimization with chaos embedded great deluge algorithm," Applied Soft Computing, vol. 12, no. 3, pp. 1055-1067, 2012.

[39] X. Zhao, Y. Zhou, and Y. Xiang, "A grouping particle swarm optimizer," Applied Intelligence, vol. 12, 2019.

[40] E. Mezura-Montes, C. A. C. Coello, and R. Landa-Becerra, "Engineering optimization using a simple evolutionary algorithm," 2003.

[41] I. Maruta, T.-H. Kim, and T. Sugie, "Fixed-structure," Automatica, vol. 45, no. 2, pp. 553-559, 2009.
[42] Y. Wang, Z. Cai, and Y. Zhou, "Accelerating adaptive trade-off model using shrinking space technique for constrained evolutionary optimization," International Journal for Numerical Methods in Engineering, vol. 77, no. 11, pp. 1501-1534, 2010.

[43] I. Brajevic and M. Tuba, "An upgraded artificial bee colony (ABC) algorithm for constrained optimization problems," Journal of Intelligent Manufacturing, vol. 24, no. 4, pp. 729740, 2013.

[44] S. Mirjalili, S. M. Mirjalili, and A. Hatamlou, "Multi-Verse Optimizer: a nature-inspired algorithm for global optimization," Neural Computing and Applications, vol. 27, no. 2, pp. 495-513, 2016.

[45] A. Kaveh and A. Dadras, "A novel meta-heuristic optimization algorithm," Advances in Engineering Software, vol. 110, no. 110, pp. 69-84, 2017.

[46] V. Kumar and D. Kumar, "An astrophysics-inspired Grey wolf algorithm for numerical optimization and its application to engineering design problems," Advances in Engineering Software, vol. 112, pp. 231-254, 2017.

[47] W. Long, J. Jiao, X. Liang, and M. Tang, "An explorationenhanced grey wolf optimizer to solve high-dimensional numerical optimization," Engineering Applications of Artificial Intelligence, vol. 68, pp. 63-80, 2018.

[48] S. Akhtar, K. Tai, and T. Ray, "A socio-behavioural simulation model for engineering design optimization," Engineering Optimization, vol. 34, no. 4, pp. 341-354, 2002.

[49] M. M. Atiqullah and S. S. Rao, "Simulated annealing and parallel processing: an implementation for constrained global design optimization," Engineering Optimization, vol. 32, no. 5, pp. 659-685, 2000.

[50] T. Ray and K. M. Liew, "Society and civilization: an optimization algorithm based on the simulation of social behavior," IEEE Transactions on Evolutionary Computation, vol. 7, no. 4, pp. 386-396, 2003.

[51] E. Mezura-Montes and B. Hernández-Ocaña, "Modified bacterial foraging optimization for engineering design," 2009.

[52] J. Zhang, C. Liang, Y. Huang, J. Wu, and S. Yang, "An effective multiagent evolutionary algorithm integrating a novel roulette inversion operator for engineering optimization," Applied Mathematics \& Computation, vol. 211, no. 2, pp. 392-416, 2009.

[53] V. S. Aragón, S. C. Esquivel, and C. A. C. Coello, “A modified version of a $\mathrm{t}$-cell algorithm for constrained optimization problems," International Journal for Numerical Methods in Engineering, vol. 84, no. 3, pp. 351-378, 2010.

[54] A. R. Hedar and M. Fukushima, "Derivative-free filter simulated annealing method for constrained continuous global optimization," 2006.

[55] E. Rashedi, H. Nezamabadi-Pour, and S. Saryazdi, "Gsa: a gravitational search algorithm," Information Sciences, vol. 179, no. 13, pp. 2232-2248, 2009.

[56] K. S. Lee and Z. W. Geem, "A new structural optimization method based on the harmony search algorithm," Computers \& Structures, vol. 82, no. 9, pp. 781-798, 2004.

[57] S. H. S. Moosavi and V. K. Bardsiri, "Satin bowerbird optimizer: a new optimization algorithm to optimize anfis for software development effort estimation," 2017.

[58] P. Savsani and V. Savsani, "Passing vehicle search (PVS): a novel metaheuristic algorithm," Applied Mathematical Modelling, vol. 40, no. 5, pp. 3951-3978, 2016.

[59] R. V. Rao, V. J. Savsani, and D. P. Vakharia, "Teaching-learningbased optimization: a novel method for constrained mechanical 
design optimization problems," Computer Aided Design, vol. 43, no. 3, pp. 303-315, 2011.

[60] Y. J. Zheng, "Water wave optimization: a new nature-inspired metaheuristic," Computers \& Operations Research, vol. 55, pp. 1-11, 2015.

[61] A. A. Heidari, S. Mirjalili, H. Faris, I. Aljarah, M. M. Mafarja, and H. Chen, "Harris hawks optimization: algorithm and applications," Future Generation Computer Systems, vol. 97, pp. 849-872, 2019.

[62] B. K. Kannan and S. N. Kramer, "An augmented Lagrange multiplier based method for mixed integer discrete continuous optimization and its applications to mechanical design," Journal of Mechanical Design, vol. 116, no. 2, pp. 405-411, 1994.

[63] Q. He and L. Wang, "An effective co-evolutionary particle swarm optimization for constrained engineering design problems," Engineering Applications of Artificial Intelligence, vol. 20, no. 1, pp. 89-99, 2007.

[64] C. A. C. Coello and N. C. Cortés, "Hybridizing a genetic algorithm with an artificial immune system for global optimization," Engineering Optimization, vol. 36, no. 5, pp. 607-634, 2004.

[65] E. Mezura-Montes and C. A. C. Coello, "An empirical study about the usefulness of evolution strategies to solve constrained optimization problems," International Journal of General Systems, vol. 37, no. 4, pp. 443-473, 2008.

[66] F. z. Huang, L. Wang, and Q. He, "An effective co-evolutionary differential evolution for constrained optimization," Applied Mathematics and Computation, vol. 186, no. 1, pp. 340-356, 2007.

[67] A. H. Gandomi, X.-S. Yang, A. H. Alavi, and S. Talatahari, "Bat algorithm for constrained optimization tasks," Neural Computing and Applications, vol. 22, no. 6, pp. 1239-1255, 2013.

[68] C. C. Coello and E. M. Montes, "Use of dominance-based tournament selection to handle constraints in genetic algorithms," Intelligent Engineering Systems Through Artificial Neural Networks, vol. 11, no. 1, pp. 177-182, 2001.

[69] K. Deb, "GeneAS: a robust optimal design technique for mechanical component design," 1997.

[70] A. Kaveh and T. Bakhshpoori, "Water evaporation optimization: a novel physically inspired optimization algorithm," Computers \& Structures, vol. 167, pp. 69-85, 2016. 